\title{
Lectures on Local Cohomology
}

\author{
Craig Huneke and Appendix 1 by Amelia Taylor
}

\begin{abstract}
This article is based on five lectures the author gave during the summer school, Interactions between Homotopy Theory and Algebra, from July 26-August 6, 2004, held at the University of Chicago, organized by Lucho Avramov, Dan Christensen, Bill Dwyer, Mike Mandell, and Brooke Shipley. These notes introduce basic concepts concerning local cohomology, and use them to build a proof of a theorem Grothendieck concerning the connectedness of the spectrum of certain rings. Several applications are given, including a theorem of Fulton and Hansen concerning the connectedness of intersections of algebraic varieties. In an appendix written by Amelia Taylor, an another application is given to prove a theorem of Kalkbrenner and Sturmfels about the reduced initial ideals of prime ideals.
\end{abstract}

\section{Contents}

1. Introduction 1

2. Local Cohomology 3

3. Injective Modules over Noetherian Rings and Matlis Duality 10

4. Cohen-Macaulay and Gorenstein rings 16

5. Vanishing Theorems and the Structure of $H_{\mathfrak{m}}^{d}(R) \quad 22$

6. Vanishing Theorems II 26

7. Appendix 1: Using local cohomology to prove a result of Kalkbrenner and Sturmfels 32

8. Appendix 2: Bass numbers and Gorenstein Rings $\quad 37$

References $\quad 41$

\section{Introduction}

Local cohomology was introduced by Grothendieck in the early 1960s, in part to answer a conjecture of Pierre Samuel about when certain types of commutative rings are unique factorization

2000 Mathematics Subject Classification. Primary 13C11, 13D45, $13 \mathrm{H} 10$.

Key words and phrases. local cohomology, Gorenstein ring, initial ideal.

The first author was supported in part by a grant from the National Science Foundation, DMS-0244405. The author thanks Robert Bruner, Anurag Singh, Emanoil Theodorescu, and the referee for valuable comments and corrections, and Ananth Hariharan for help with LaTex. 
domains. ${ }^{1}$ Local cohomology has since become an indepensable tool and is the subject of much research. This article will be concentrating on several applications of local cohomology, whose proofs force the development of most of the basic material concerning local cohomology. All rings in this article will be commutative rings with identity, and usually will be Noetherian as well. We assume familarity with basic commutative algebra, including the notions of height, dimension, depth, and primary decomposition.

Among many other attributes, local cohomology allows one to answer many seemingly difficult questions. A good example of such a problem, where local cohomology provides a partial answer, is the question of how many generators ideals have up to radical. In general, if $J$ is an ideal of a ring $R$, the radical of $J$ is the ideal

$$
\sqrt{J}=\left\{x \in R \mid x^{m} \in J \text { for some } m\right\} .
$$

We say an ideal $J$ is generated up to radical by $n$ elements if there exist $x_{1}, \ldots, x_{n} \in J$ such that $\sqrt{J}=\sqrt{\left(x_{1}, \ldots, x_{n}\right)}$. For example, the ideal $J \subseteq k[x, y]$ generated by $x^{2}, x y, y^{2}$ is generated up to radical by the two elements $x^{2}, y^{2}$. Recall that the radical of an ideal $I$ is the intersection of all primes ideals which contain $I$. Hilbert's famous Nullstellensatz says even more holds in the case $R$ is a polynomial ring over a field: the radical of $I$ is the intersection of all maximal ideals containing $I$.

Given an ideal $I$ what is the least number of elements needed to generate it up to radical? A particular example of this problem is the following: let $R=k[x, y, u, v]$ be a polynomial ring in four variables over the field $k$. Consider the ideal $I=(x u, x v, y u, y v)$. This ideal is its own nilradical, i.e. $I=\sqrt{I}$. The four given generators of $I$ are minimal. On the other hand, it can be generated not on the nose, but up to radical, by the three elements $x u, y v, x v+y u$. This holds since $(x v)^{2}=x v(x v+y u)-(x u)(y v) \in(x u, y v, x v+y u)$. Are there two elements which generate it up to radical? Could there even be one element which generates $I$ up to radical?

The answer to the last question is no, there cannot be just one element generating the ideal $I$ up to radical, due to an obstruction first proved by Krull, namely the height of the ideal. Krull's famous height theorem states:

TheOrem 1.1. (Krull's Height Theorem) Let $R$ be a Noetherian ring and $I=\left(x_{1}, \ldots, x_{n}\right)$ be an ideal generated by $n$ elements. If $P$ is a minimal prime over $I$, then the height of $P$ is at most $n$. In particular, if an ideal $I$ is generated up to radical by $n$ elements, then the height of $I$ is at most $n$.

In the example we are considering, the height of $I$ is two as it is the product of the two height two ideals $(x, y)$ and $(u, v)$. Krull's height theorem implies that two is the smallest number of polynomials which could generate $I$ up to radical. This still begs the question, are there two polynomials $F, G \in I$ such that $\sqrt{(F, G)}=I$ ? Trying to find two such polynomials $F, G$ by some type of random search would be hard, if not impossible. Of course if there are no such polynomials, no search would find them(!), but even if two such polynomials do exist, it is likely no random search would find them. The problem is that these polynomials would normally be extremely special, so that writing down general polynomials in $I$ would not work. ${ }^{2}$ Instead, we would like to find, in some cohomology theory, an obstruction to being generated up to radical by two elements. Local cohomology provides such an obstruction. To a $\operatorname{ring} R$ and ideal $J$, we'll associate for $i \geq 0$ modules $H_{J}^{i}(R)$ with the properties that

(i) $H_{J}^{i}(R)=H_{\sqrt{J}}^{i}(R)$, and

\footnotetext{
${ }^{1}$ Specifically, Samuel made the following conjecture, subsequently proved by Grothendieck [15]: Let $R$ be a Noetherian local ring and $\widehat{R}$ its completion with respect to the maximal ideal. If $\widehat{R}$ is a complete intersection and for each prime ideal $P$ of $R$ of height $\leq 3, R_{P}$ is a UFD, then $R$ is a UFD.

${ }^{2}$ It turns out that the least number of general elements required to generate $I$ up to radical in a local Noetherian ring is exactly what is called the analytic spread of $I$. See [39].
} 
(ii) if $J$ is generated by $k$-elements, then $H_{J}^{i}(R)=0$ for all $i>k$.

Finally, for $I=(x u, x v, y u, y v)$, we'll prove that $H_{I}^{3}(R) \neq 0$, and therefore $I$ cannot be generated up to radical by two elements. See Example 5.5.

I find that I always learn things best when there is clear direction and motivation. Aiming at a theorem I want to understand helps me learn. I'm hoping the same will be true for the reader, and for this reason this article will be aimed at a theorem of Grothendieck concerning the connectedness of certain algebraic sets, a result which has beautiful applications to intersections of projective varieties, and to simplicial complexes associated to initial ideals. Almost everything proved in this article is necessary to get to the theorem of Grothendieck and its applications. On the other hand, some sections are intended as extra information for the reader, with directions of where to find more information. These sections can in general be skipped by a reader who prefers to see proofs, and nothing but the proofs. The reader will be warned if a section is not used later in the article.

One of the applications of the theorem of Grothendieck which will be proved is the following theorem, due to Fulton and Hansen:

THEOREM 1.2. ([13]) Let $k$ be an algebraically closed field and suppose that $X \subseteq \mathbb{P}_{k}^{n}$ and $Y \subseteq \mathbb{P}_{k}^{n}$ are algebraic varieties (i.e., reduced and irreducible closed algebraic sets). If $\operatorname{dim}(X)+\operatorname{dim}(Y)>n$, then $X \cap Y$ is connected.

An appendix written by Amelia Taylor will give another somewhat surprising application of our basic connectedness theorem to Gröbner bases of prime ideals.

The article is divided into five sections, each roughly the topic of one of the original five lectures. Each section comes with a few exercises. Additional material is sometimes added at the end of the section, usually without proofs, for extra information. There are two appendices: the first written by Amelia Taylor, giving her reworking of a theorem of Kalkbrenner and Sturmfels, and the second giving a more "classical" treatment of Gorenstein rings. The first section gives basic definitions and results. The second section develops the theory of Matlis duality. The third section provides an introduction to Cohen-Macaulay and Gorenstein rings, and proves local duality. The fourth section proves some important vanishing theorems concerning local cohomology, while the fifth section proves an especially important theorem due to Hartshorne and Lichtenbaum, and gives a proof of the theorem of Fulton and Hansen stated above.

\section{Local Cohomology}

We begin by recalling equivalent definitions of injective modules:

Proposition 2.1. Let $R$ be a (commutative) ring, $E$ an $R$-module. Then the following are equivalent:

(1) Let $M \subseteq N$ be an inclusion of R-modules. Every homomorphism from $M$ to $E$ extends to a homomorphism from $N$ to $E$.

(2) (Baer's Criterion) Let I be an ideal in R. Every homomorphism from $I$ to $E$ extends to a homomorphism from $R$ to $E$.

(3) $\operatorname{Hom}_{R}(, E)$ preserves short exact sequences (contravariantly).

When any of these equivalent conditions occur, we say that $E$ is an injective $R$-module. The equivalence of (1), (2) and (3) is straightforward. Another equivalent property is the following: a module $E$ is injective if and only if whenever $E \subseteq M$, this inclusion splits, i.e., there exists a homomorphism $f: M \rightarrow E$ such that the inclusion of $E$ in $M$ composed with $f$ is the identity on $M$. The fact that injective modules split from every larger module is elementary provided $E$ satisfies any of (1), (2), or (3). The other direction, that this property characterizes injective modules, needs 
some proof. The clearest proof follows from the fact that every module $E$ can be imbedded into an injective module $I$. Since $E$ splits out of $I$ by assumption, $E$ is injective, since it is elementary that direct summands of injective modules are injective.

It turns out that there is a smallest injective in a rather precise sense containing a given module, up to isomorphism. To describe this module we need a definition:

Definition 2.2. If $N \subseteq M$ are $R$-modules, then $M$ is said to be essential over $N$ if every non-zero submodule $T$ of $M$ has a non-zero intersection with $N$.

Proposition 2.3. Let $R$ be a ring and $M \subseteq E$ be $R$-modules. The following conditions are equivalent.

(1) $E$ is a maximal essential extension of $M$, i.e., if $E \subseteq F$ and $F$ is also essential over $M$, then $E=F$.

(2) $E$ is a minimal injective containing $M$, i.e., if $M \subseteq I \subseteq E$ and $I$ is injective, then $I=E$.

(3) $E$ is an injective module and is an essential extension of $M$.

DeFINITION 2.4. A module $E$ with any (and hence all) of the above properties is called an injective hull of $M$ and is denoted by $E_{R}(M)$.

An injective hull $E_{R}(M)$ of $M$ is unique up to isomorphism. The injective hull depends not only on the module $M$ but also upon the ring $R$. Thus, in general, $E_{R}(M) \nsucc E_{R / I}(M)$ for an ideal $I$ with $I M=0$.

There is an important point which is somewhat hidden in the description of an injective hull of a module $M$ as a "maximal" essential extension of $M$ which we make more precise and will use without further comment. Namely, suppose that we fix an injective hull $E$ of a module $M$, and have an essential extension $M \subseteq N$ of $M$. Then this inclusion induces an embedding of $N$ in $E$ compatible with the inclusion of $M$ into $E$. The injectivity of $E$ gives the existence of a homomorphism $f: N \rightarrow E$ extending the inclusion of $M \subseteq N$, and $f$ is necessarily injective because if the kernel of $f$, say $K$, is nonzero, then it would have a nonzero intersection with $M$, contradicting the fact that $M$ is embedded in $E$. This means that $E_{R}(M)$ literally contains an isomorphic copy of every essential extension of $M$. It is truly a maximal essential extension.

To give some examples of injective modules, it helps to focus on one of their important properties, namely that they are divisible.

Definition 2.5. Let $M$ be an $R$-module. We say that $M$ is divisible if whenever $x$ is a nonzerodivisor in $R$ and $u \in M$, there exists an element $v \in M$ (not necessarily unique) such that $x v=u$.

Remark 2.6. Every injective module is divisible. For let $E$ be an injective $R$-module, and let $u \in E$. Apply the injective property (1) of Proposition 2.1 for $E$ to the map $\mu_{x}$ from $R$ to $R$ which is given by multiplication by $x$, and the map $f$ of $R$ to $E$ sending 1 to $u$. We can extend the latter map to a homomorphism $g: R \rightarrow E$, so that $g \circ \mu_{x}=f$. Evaluating these maps at 1 gives that $u=f(1)=g\left(\mu_{x}(1)\right)=g(x)=x g(1)$. Set $v=g(1)$.

How close is divisibility to injectivity? Not that close, except in a few cases. One case where they are equivalent is if $R$ is a PID, a principal ideal domain. This follows easily from Baer's Criterion (see Exercise 3). Another situation is that any module over an integral domain which is both torsion-free and divisible is injective. See Exercise 1. We can use these cases to give some examples of injective modules:

Example 2.7. If $R$ is a domain, then $E_{R}(R)=K$ the quotient field of $R$. This follows from two facts: first if $W$ is a multiplicatively closed set of non-zero-divisors on a module $M$ then $M_{W}$ 
is an essential extension of $M$. Secondly, any module $E$ over a domain $R$ which is both divisible and torsion-free is necessarily injective. Thus $K$ is both essential over $R$ and injective, so by Proposition 8.1 (3) is therefore an injective hull of $R$.

ExAmple 2.8. If $R$ is a principal ideal domain then an $R$-module $E$ is injective if and only if $E$ is divisible. In particular, if $K$ is the fraction field of $R$, then both $K$ and $K / R$ are divisible, hence injective. Thus there is an exact sequence,

$$
0 \rightarrow R \rightarrow K \rightarrow K / R \rightarrow 0
$$

in which all terms but the first are injective $R$-modules. Such a sequence is called an injective resolution of $R$.

Definition 2.9. An injective resolution $E^{*}$ of an $R$-module $M$ is an exact sequence:

$$
0 \longrightarrow M \longrightarrow E^{0} \stackrel{\varphi_{0}}{\longrightarrow} E^{1} \stackrel{\varphi_{1}}{\longrightarrow} \ldots \rightarrow E^{n} \stackrel{\varphi_{n}}{\longrightarrow} E^{n+1} \rightarrow \ldots,
$$

where each $E^{i}$ is an injective $R$-module. An injective resolution is called a minimal injective resolution if $E^{0}$ is an injective hull of $M$, and for all $i \geq 0, E^{i+1}$ is an injective hull of $\operatorname{Ker}\left(\varphi_{i+1}\right)=$ $\operatorname{Im}\left(\varphi_{i}\right)$.

It is not difficult to show that up to an isomorphism of exact sequences, a minimal injective resolution is unique.

We now come to the basic definition of this article. Let $R$ be a commutative ring, $I$ an ideal of $R$, and $M$ an $R$-module.

Definition 2.10. Set

$$
\Gamma_{I}(M)=\left\{x \in M \mid \text { there exists } n \in \mathbb{N} \text { such that } I^{n} u=0\right\},
$$

and let $H_{I}^{i}(\quad)$ be the ith right derived functor of $\Gamma_{I}$.

Recall one computes these modules by taking an injective resolution of $M$, applying $\Gamma_{I}$, and taking cohomology. Since $\Gamma_{I}$ is left exact, we have that

$$
H_{I}^{0}(M)=\Gamma_{I}(M) \text {. }
$$

Observe that if $J$ is another ideal with the same nilradical as $I$, then $\Gamma_{I}$ and $\Gamma_{J}$ are the same functor. Hence $H_{J}^{i}(M)=H_{I}^{i}(M)$ for all $i$ and for all $R$-modules $M$. This is a critically important property of local cohomology.

Another elementary, but important, property of the local cohomology modules is that every element in $H_{I}^{i}(M)$ is killed by a power of $I$. This follows at once from the definition.

Still another property is that given a short exact sequence of $R$-modules, $0 \rightarrow N \rightarrow M \rightarrow L \rightarrow 0$, there is an induced long exact sequence on local cohomology,

$$
0 \rightarrow H_{I}^{0}(N) \rightarrow H_{I}^{0}(M) \rightarrow H_{I}^{0}(L) \rightarrow H_{I}^{1}(N) \rightarrow H_{I}^{1}(M) \rightarrow \ldots
$$

This follows by the usual yoga of derived functors and we will not prove it in this article.

We often refer to the local cohomology modules as the local cohomology of $M$ with support in $I$. This is an abuse of notation, but the justification is the following: the functor $\Gamma_{I}(M)$ identifies a submodule of $M$ whose elements are supported on the closed set $V(I) \subseteq \operatorname{Spec}(R)$. This means that if $P \in \operatorname{Spec}(R)$ and $P$ does not contain $I$, then $\left(\Gamma_{I}(M)\right)_{P}=0$. This holds since the elements in $\Gamma_{I}(M)$ are killed by powers of $I$, so that if we invert some element of $I$, they must become 0 .

EXAmple 2.11. Let $p$ be a prime number. We compute $H_{I}^{i}(\mathbb{Z})$, where $I$ is the ideal generated by $p$. Since $\mathbb{Z}$ is a PID, all divisible modules are injective, and an injective resolution of $\mathbb{Z}$ is given by

$$
0 \rightarrow \mathbb{Z} \rightarrow \mathbb{Q} \rightarrow \mathbb{Q} / \mathbb{Z} \rightarrow 0
$$


The functor $\Gamma_{I}$ simply computes the $p^{n}$-torsion for all $n$. Applying this functor to the injective resolution, there is only one non-vanishing term, namely $\Gamma_{I}(\mathbb{Q} / \mathbb{Z})$, which sits in cohomological degree 1. Thus all the local cohomology vanishes except for $H_{I}^{1}(\mathbb{Z})$, and this is isomorphic to the $p$-torsion in $\mathbb{Q} / \mathbb{Z}$. By the unique factorization property, this module can be identified with $\mathbb{Z}\left[p^{-1}\right] / \mathbb{Z}$, where $\mathbb{Z}\left[p^{-1}\right]$ is the ring of rational numbers whose denominators are a power of $p$.

EXAmple 2.12. A very similar example which is more in the direction of these notes is the computation of $H_{I}^{i}(M)$, where $R=k[x], k$ a field, $I=(x)$, and $M$ is a finitely generated $R$-module. By the structure theorem for PIDs, $M$ is a direct sum of cyclic modules. As local cohomology commutes with direct sums, it suffices to compute the local cohomology of $R /(g)$ for some $g \in R$. We first compute the local cohomology of $R$ itself, i.e. when $g=0$.

As above, since $R$ is a PID, any divisible module is injective. The injective hull of $R$ is the fraction field $K=k(x)$, and since $K / R$ is divisible, it is injective. Thus an injective resolution is given by

$$
0 \rightarrow R \rightarrow K \rightarrow K / R \rightarrow 0
$$

We apply $\Gamma_{I}$ and compute the local cohomology as the cohomology of the complex

$$
0 \rightarrow \Gamma_{I}(K) \rightarrow \Gamma_{I}(K / R) \rightarrow 0 .
$$

It follows at once that $H_{I}^{0}(R)=0$ and $H_{I}^{j}(R)=0$ for all $j>1$. We can also identify $\Gamma_{I}(K / R)=$ $H_{I}^{1}(R)$. As above, the unique factorization property shows that

$$
H_{I}^{1}(R) \cong R\left[x^{-1}\right] / R=k\left[x, x^{-1}\right] / k[x] .
$$

This module has a $k$-basis of elements $\frac{1}{x^{n}}$, where $n \geq 1$. Multiplication by $x$ shifts these basis elements in the normal way except at the 'end': $x \cdot \frac{1}{x}=0$.

To compute $H_{I}^{i}(R /(g))$ for $g \neq 0$ we use the short exact sequence,

$$
0 \rightarrow R \stackrel{g}{\rightarrow} R \rightarrow R /(g) \rightarrow 0 .
$$

This short exact sequence induces a long exact sequence on cohomology, with the maps from $H_{I}^{i}(R)$ to $H_{I}^{i}(R)$ given by multiplication by $g$. Since there is only one non-vanishing local cohomology for $R$, we get a four-term sequence:

$$
0 \rightarrow H_{I}^{0}(R /(g)) \rightarrow H_{I}^{1}(R) \stackrel{g}{\rightarrow} H_{I}^{1}(R) \rightarrow H_{I}^{1}(R /(g)) \rightarrow 0
$$

As every element of $H_{I}^{1}(R)$ is killed by a power of $I$, if $h$ is relatively prime to $x$, then $h$ must act as a unit on $H_{I}^{1}(R)$ since there exist $a, b \in R$ such that $a h=1-b x$, and $1-b x$ acts as a unit on this module. Writing $g=x^{n} h$ where $(h, x)=1$, it follows that $H_{I}^{0}(R /(g))$ is the kernel of multiplication by $x^{n}$ on $H_{I}^{1}(R)$, and $H_{I}^{1}(R /(g))$ is the cokernel of multiplication by $x^{n}$. The set of elements in $H_{I}^{1}(R)$ annihilated by $x^{n}$ is generated by $\frac{1}{x^{n}}$ and is thus isomorphic to $R /\left(x^{n}\right)$. Since $H_{I}^{1}(R)=R\left[x^{-1}\right] / R$, this module is divisible by $R$, and thus the cokernel is 0 .

We summarize these results: if $g=0$, then $H_{I}^{i}(R)=0$ for all $i \neq 1$, and $H_{I}^{1}(R) \cong R\left[x^{-1}\right] / R$. If $g \neq 0$, then writing $g=x^{n} h$, where $x$ does not divide $h$, we have that $H_{I}^{i}(R /(g))=0$ for all $i \neq 0$ and $H_{I}^{0}(R /(g)) \cong R /\left(x^{n}\right)$.

2.1. Two Other Important Ways to Think About Local Cohomology. There are two very important alternative definitions of local cohomology when the base $\operatorname{ring} R$ is Noetherian. We will not prove the equivalence except to note that if one has two sequences of cohomology functors $F^{i}$ and $G^{i}$ which induce functorial long exact sequences given a short exact sequence of modules, which agree for $i=0$, and such that $F^{i}(E)=G^{i}(E)=0$ for all $i>0$ whenever $E$ is injective, then it is a straightforward induction to prove that $F^{i}(M) \cong G^{i}(M)$ functorially for all $i$. 
For the first alternate definition, we rewrite $\Gamma_{I}(M)$ as the direct union of the submodules $0:{ }_{M} I^{n}$. In fact if $\left\{I_{n}\right\}$ is any nested system of ideals which are cofinal with the powers of $I$, it is clear that

$$
\Gamma_{I}(M)=\cup_{n}\left(0:_{M} I_{n}\right) .
$$

Identify $\left(0:_{M} I_{n}\right)=\operatorname{Hom}_{R}\left(R / I_{n}, M\right)$, so that

$$
H_{I}^{0}(M)=\Gamma_{I}(M)=\underline{\lim _{\longrightarrow}} \operatorname{Hom}_{R}\left(R / I_{n}, M\right)
$$

and it follows easily as described above from the long exact sequences associated to the functors Hom and $\Gamma$ that this identification induces an identification

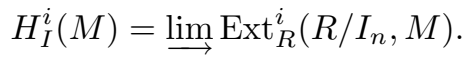

We will use the full strength of this identification when we prove what is called the HartshorneLichtenbaum vanishing theorem (HLVT for short). There are two filtrations that are often good to consider. A first important filtration is the symbolic power filtration, $\left\{I^{(n)}\right\}$. Recall that $I^{(n)}$ is the pullback of $I^{n}$ after inverting all elements not in any minimal prime of $I$. A second important filtration, in positive characteristic, is the Frobenius power filtration, $\left\{I^{\left[p^{e}\right]}\right\}$, where $I^{\left[p^{e}\right]}$ is the ideal generated by all the $p^{e}$ th powers of elements of $I$. Although the Frobenius powers are always cofinal with the powers of $I$ (provided $I$ is finitely generated), the symbolic powers need not be cofinal in general.

The second identification is a bit more subtle. For $x \in R$, let $K^{\bullet}(x ; R)$ denote the complex $0 \rightarrow R \rightarrow R_{x} \rightarrow 0$, graded so that the degree 0 piece of the complex is $R$, and the degree 1 is $R_{x}$. Here an elsewhere, we write $R_{x}$ for the localization of $R$ at the multiplicatively closed set $\left\{x^{n}\right\}$, i.e., $R_{x}=$ $R\left[x^{-1}\right]$. If $x_{1}, \ldots, x_{n} \in R$, let $K^{\bullet}\left(x_{1}, x_{2}, \ldots, x_{n} ; R\right)$ denote the complex $K^{\bullet}\left(x_{1} ; R\right) \otimes_{R} \ldots \otimes_{R} K^{\bullet}\left(x_{n} ; R\right)$, where in general recall that if $\left(C^{\bullet}, d_{C}\right)$ and $\left(D^{\bullet}, d_{D}\right)$ are complexes, then the tensor product of these complexes, $\left(C \otimes_{R} D, \Delta\right)$ is by definition the complex whose ith graded piece is $\sum_{j+k=i} C_{j} \otimes D_{k}$ and whose differential $\Delta$ is determined by the map from $C_{j} \otimes D_{k} \rightarrow\left(C_{j+1} \otimes D_{k}\right) \oplus\left(C_{j} \otimes D_{k+1}\right)$ given by $\Delta(x \otimes y)=d_{C}(x) \otimes y+(-1)^{k} x \otimes d_{D}(y)$.

The modules in this Koszul cohomology complex are

$$
0 \rightarrow R \rightarrow \oplus \sum_{i} R_{x_{i}} \rightarrow \oplus \sum_{i<j} R_{x_{i} x_{j}} \rightarrow \ldots \rightarrow R_{x_{1} x_{2} \cdots x_{n}} \rightarrow 0
$$

where the differentials are the natural maps induced from localization, but with signs attached. If $M$ is an $R$-module, we set $K^{\bullet}\left(x_{1}, x_{2}, \ldots, x_{n} ; M\right)=K^{\bullet}\left(x_{1}, x_{2}, \ldots, x_{n} ; R\right) \otimes_{R} M$. We denote the cohomology of $K^{\bullet}\left(x_{1}, x_{2}, \ldots, x_{n} ; M\right)$ by $H^{i}\left(\underline{x}^{\infty} ; M\right)$. The second identification is the following:

Proposition 2.13. Let $R$ be a commutative Noetherian ring, $I$ an ideal, and $M$ an $R$-module. Suppose that $\sqrt{I}=\sqrt{\left(x_{1}, \ldots, x_{n}\right)}$. Then for all $i$,

$$
H_{I}^{i}(M) \cong H^{i}\left(\underline{x}^{\infty} ; M\right),
$$

and this isomorphism is functorial.

Proof. Since local cohomology depends on $I$ only up to radical, without loss of generality, $I$ can be assumed to be generated by the $x_{i}$. The Koszul cohomology does induce functorial long exact sequences given a short exact sequence of modules. We prove that $H^{0}\left(\underline{x}^{\infty} ; M\right)=H_{I}^{0}(M)$. By definition, $H^{0}\left(\underline{x}^{\infty} ; M\right)$ is the homology of the sequence

$$
0 \rightarrow M \rightarrow M_{x_{1}} \oplus \ldots \oplus M_{x_{n}} .
$$

An element $y \in M$ goes to zero if and only if it goes to zero in each localization, if and only if for each $i$ there is an integer $n_{i}$ such that $y x_{i}^{n_{i}}=0$, if and only if there is an $N$ such that $y I^{N}=0$, if and only if $y \in H_{I}^{0}(M)$. To finish the proof one needs to prove that $H^{i}\left(\underline{x}^{\infty} ; E\right)=0$ for all injective 
$R$-modules $E$, and for all $i>0$. This follows because, as we shall see in the next section, on each indecomposable summand of $E$, each $x_{i}$ acts either nilpotently or as a unit. This is easily seen to force the higher cohomology to be zero.

Let's return to the first example, $R=k[X]$ and $I=(X)$. Given an $R$-module $M$, we can compute the local cohomology of $M$ as the cohomology of the sequence,

$$
0 \rightarrow M \rightarrow M_{X} \rightarrow 0
$$

which is exactly what we found by hand.

This last avatar of local cohomology is in many ways the most powerful. At the moment, we use it to prove two important properties of local cohomology.

Proposition 2.14. Let $R$ be a Noetherian ring, $I$ and ideal and $M$ and $R$-module. Let $\varphi: R \rightarrow$ $S$ be a homomorphism and let $N$ be an $S$-module.

(1) If $\varphi$ is flat, then $H_{I}^{j}(M) \otimes_{R} S \cong H_{I S}^{j}\left(M \otimes_{R} S\right)$. In particular, local cohomology commutes with localization and completion.

(2) (Independence of Base) $H_{I}^{j}(N) \cong H_{I S}^{j}(N)$, where the first local cohomology is computed over the base ring $R$.

Proof. Choose generators $x_{1}, \ldots, x_{n}$ of $I$. The first claim follows at once from the fact that $\left.K^{\bullet}\left(x_{1}, \ldots, x_{n} ; M\right) \otimes_{R} S=K^{\bullet}\left(\varphi\left(x_{1}\right), \ldots, \varphi\left(x_{n}\right) ; M\right) \otimes_{R} S\right)$, and that $S$ is flat over $R$, so that the cohomology of $K^{\bullet}\left(x_{1}, \ldots, x_{n} ; M\right) \otimes_{R} S$ is the cohomology of $K^{\bullet}\left(x_{1}, \ldots, x_{n} ; M\right)$ tensored over $R$ with $S$.

The second claim follows from the fact that

$$
\begin{gathered}
K^{\bullet}\left(x_{1}, \ldots, x_{n} ; N\right)=K^{\bullet}\left(x_{1}, \ldots, x_{n} ; R\right) \otimes_{R} N=\left(K^{\bullet}\left(x_{1}, \ldots, x_{n} ; R\right) \otimes_{R} S\right) \otimes_{S} N \\
=K^{\bullet}\left(\varphi\left(x_{1}\right), \ldots, \varphi\left(x_{n}\right) ; S\right) \otimes_{R} N=K^{\bullet}\left(\varphi\left(x_{1}\right), \ldots, \varphi\left(x_{n}\right) ; N\right) .
\end{gathered}
$$

We can apply both of these alternate ways to see that computing local cohomology in the support of the maximal ideal of a local ring is the same as computing it over the completion. To be specific:

Proposition 2.15. Let $(R, \mathfrak{m}, k, E)$ be a Noetherian local ring of dimension $d$, and let $M$ be a finitely generated $R$-module. For all $i \geq 0, H_{\mathfrak{m}}^{i}(M) \cong H_{\widehat{\mathfrak{m}}}^{i}(\widehat{M})$.

Proof. By the Independence of Base, $H_{\widehat{\mathfrak{m}}}^{i}(\widehat{M}) \cong H_{\mathfrak{m} \widehat{R}}^{i}(\widehat{M})$. Since $H_{\mathfrak{m}}^{i}(M) \cong \underline{\lim _{\longrightarrow}} \operatorname{Ext}_{R}^{i}\left(R / \mathfrak{m}^{n}, M\right)$, and because $\widehat{R}$ is flat over $R$, we see that

$$
H_{\mathfrak{m} \widehat{R}}^{i}(\widehat{M}) \cong H_{\mathfrak{m} \widehat{R}}^{i}\left(M \otimes_{R} \widehat{R}\right) \cong H_{\mathfrak{m}}^{i}(M) \otimes_{R} \widehat{R} \cong \lim _{\longrightarrow} \operatorname{Ext}_{R}^{i}\left(R / \mathfrak{m}^{n}, M\right) \otimes_{R} \widehat{R} \cong \lim _{\longrightarrow} \operatorname{Ext}_{R}^{i}\left(R / \mathfrak{m}^{n}, M\right),
$$

the last isomorphism following as these Ext modules are killed by a power of the maximal ideal.

\subsection{The Graded Case.}

Definition 2.16. A ring $R$ is graded if we can write $R=\oplus_{i \geq 0} R_{i}$ as abelian groups under addition in such a way that the multiplication map preserves the grading, i.e., $R_{i} \cdot R_{j} \subseteq R_{i+j}$.

Since $R_{0} \cdot R_{0} \subseteq R_{0}$, it follows that $R_{0}$ is a ring itself (necessarily containing 1 ), and as $R_{0} \cdot R_{j} \subseteq R_{j}$, each $R_{j}$ is an $R_{0}$-module. We say a non-zero element $x \in R$ is homogeneous if $x \in R_{j}$ for some $j$. In this case we call $j$ the degree of $x$. Of course, 0 is in every $R_{j}$. 
DeFinition 2.17. Let $R$ be a graded ring and let $I$ be an ideal of $R$. We say $I$ is homogeneous if either of the following two equivalent conditions is satisfied: (1) $I$ is generated as an ideal by homogeneous elements. (2) If $f=f_{i}+\ldots+f_{j} \in I$, where $i \leq j$ and $f_{l} \in R_{l}$ for $i \leq l \leq j$, then each $f_{l} \in I$ for $i \leq l \leq j$.

We say that a graded ring $R$ is standard graded if $R_{0}$ is a field, and $R$ is generated over $R_{0}$ by $R_{1}$, i.e., $R=R_{0}\left[R_{1}\right]$. This means that $R_{j}=\left(R_{1}\right)^{j}$ for all $j \geq 1$. We also usually insist that $R_{1}$ is a finitely generated $R$-module. If $R_{1}=R_{0} u_{1}+\ldots+R_{0} u_{n}$ and $R$ is standard graded, then we can map a polynomial ring $R_{0}\left[x_{1}, \ldots, x_{n}\right]$ onto $R$ by sending $x_{i}$ to $u_{i}$. This map is homogeneous, i.e., preserves degrees, provided we give $x_{i}$ degree 1 . In this case we can write $R \cong R_{0}\left[x_{1}, \ldots, x_{n}\right] / I$ for some homogeneous ideal $I$ of $R$. All such $R$ are Noetherian by the Hilbert Basis Theorem.

DeFinition 2.18. Let $R$ be a graded ring and let $M$ be an $R$-module. We say that $M$ is graded if as an abelian group, $M=\oplus_{i \in \mathbb{Z}} M_{i}$, and the action of $R$ on $M$ preserves the grading, i.e., $R_{j} \cdot M_{k} \subseteq M_{j+k}$.

Although we have defined a graded ring to have only nonnegative degrees, a module is allowed to have both positive and negative degree pieces. Of course, the theory can be developed for rings without the restriction to nonnegative degree, but for this article we do not need this more general theory. Suppose that $R$ is graded, $M$ is a graded $R$-module, and $I$ is homogeneous. Choose homogeneous elements $x_{1}, \ldots, x_{n}$ which generate $I$ up to radical. Whenever $y$ is a form of degree $t$, the module $M_{y}$ inherits a natural grading given as follows: if $x \in M$ has degree $d$, then $\frac{x}{y^{n}}$ is given degree $d-n t$. In this way the modules in the complex $K^{\bullet}\left(x_{1}, x_{2}, \ldots, x_{n} ; M\right)$ are all graded, and so the cohomology modules are also graded. What is not obvious, but nonetheless is true, is that this grading is independent of the choice of $x_{i}$. In our example, the local cohomology module $H_{(x)}^{1}(R)=k\left[x, x^{-1}\right] / k[x]$ has the grading where the element $\frac{1}{x^{n}}$ has degree $-n$. This module has no elements in nonnegative degrees, but does have an element in degree -1 , namely $\frac{1}{x}$. This element is annihilated by the entire homogeneous maximal ideal of $R$; this is always true of elements of maximal degree in a module, since multiplication by elements of the maximal ideal of $R$ increase the degree. Recall that the socle of a module $M$ is the set of elements in $M$ annihilated by the maximal ideal (or the maximal homogeneous ideal in the graded case). This set is a vector space over the residue field of the maximal ideal. The module $H_{(x)}^{1}(R)$ has a 1-dimensional socle, generated in degree -1 .

Consider the local cohomology module $H_{(x, y)}^{2}(k[x, y])$ for another example. We use the Koszul homology to calculate this group; it is the cokernel of the map

$$
k\left[x, y, x^{-1}\right] \oplus k\left[x, y, y^{-1}\right] \rightarrow k\left[x, y, x^{-1}, y^{-1}\right] .
$$

The terms in this Koszul cohomology sequence can be easily pictured. The ring itself has a $k$-basis of monomials in $x$ and $y$, which can be realized as the lattice points in the first quadrant. Inverting $x$ adds all the lattice points in the second quadrant, inverting $y$ adds the lattice points in the fourth quadrant, and inverting both adds all the integral lattice points in the plane. The cokernel is then easily identified with the points in the third quadrant, basically the ring $R$ turned upside down. It also has a 1-dimensional socle, namely the element $x^{-1} y^{-1}$, which lives in degree -2 , the top nonvanishing degree of the local cohomology.

This picture continues in higher dimension. If $R=k\left[x_{1}, \ldots, x_{n}\right]$ is a polynomial ring over a field $k$, then the highest local cohomology of $R, H_{\mathfrak{m}}^{n}(R) \cong k\left[x_{1}^{-1}, \ldots, x_{n}^{-1}\right]$, where multiplication by $x_{i}$ acts as usual by shifting indices except that $x_{i} \cdot x_{i}^{-1}=0$. This is $R$ turned upside down. In this module, there is a unique (up to unit) element of highest degree, namely $x_{1}^{-1} \cdots x_{n}^{-1}$, which has degree $-n$. This element generates the socle of $H_{\mathfrak{m}}^{n}(R)$. 


\subsection{Exercises.}

EXERCISE 1. Prove that a torsion-free divisible module over a domain is injective.

Exercise 2. Prove Baer's criterion, Proposition 2.1 (2), i.e. prove the equivalence of (2) with Proposition 2.1 (1).

EXERCISE 3. Over a PID, prove a module $E$ is injective if and only if $E$ is divisible.

Exercise 4. Prove that $\Gamma_{I}$ is left exact.

EXERCISE 5. Let $n$ be an integer. Compute $H_{(n)}^{i}(\mathbb{Z})$.

EXERCISE 6. Prove the isomorphism $H_{\mathfrak{m}}^{n}(R) \cong k\left[x_{1}^{-1}, \ldots, x_{n}^{-1}\right]$ given in the text above.

EXERCISE 7. Find the local cohomology of a finite abelian group $G$ with respect to $I=(p), p$ a prime number.

EXERCISE 8. Let $I$ be an injective module, and suppose that $I \cong E_{1} \oplus E_{2}$. Prove that both $E_{i}$ are injective.

\section{Injective Modules over Noetherian Rings and Matlis Duality}

This section develops basic material concerning the structure of injective modules over Noetherian rings culminating in a proof of Matlis duality.

THEOREM 3.1. Let $R$ be a Noetherian ring. An $R$-module $E$ is an indecomposable injective module if and only if $E \cong E_{R}(R / p)$ for some prime $p$ of $R$. Every finitely generated submodule $M$ of $E_{R}(R / p)$ has only $p$ as an associated prime. Every injective module is the direct sum of indecomposable injective modules.

Proof. We first prove that $E=E_{R}(R / p)$ is indecomposable. If not, write $E=E_{1} \oplus E_{2}$, and let $I_{i}=E_{i} \cap(R / p)$. Since $E$ is essential over $R / p, I_{i} \neq 0$. Then $I_{1} I_{2} \neq 0$ since $R / p$ is a domain, and hence $I_{1} \cap I_{2} \neq 0$. This forces $E_{1} \cap E_{2} \neq 0$ which is a contradiction.

We claim that $p$ is the only associated prime of $E_{R}(R / p)$. That it to say, if $q$ is a prime and $R / q$ embeds in $E_{R}(R / p)$, then $q=p$. Suppose that there is an embedding $R / q \subseteq E_{R}(R / p)$, and let $E=E_{R}(R / q)$. Since $E_{R}(R / q)$ is essential over $R / q$ it follows that $E_{R}(R / q)$ also embeds in $E_{R}(R / p)$, and therefore is a direct summand of $E_{R}(R / p)$. As $E_{R}(R / p)$ is indecomposable this shows that $E \cong E_{R}(R / p)$. We have established an isomorphism $E_{R}(R / q) \cong E_{R}(R / p)$. Then this module is an essential extension of both $R / q$ and $R / p$. The intersection of these submodules must be nonzero and a submodule of each. However, the intersection is annihilated by $p+q$. Since the annihlator of an arbitrary nonzero element of $R / p$ is $p$, this forces $q \subseteq p$. Similarly $p \subseteq q$, so that $p=q$.

Next suppose that $E$ is an arbitrary indecomposable injective. Let $M$ be any finitely generated submodule of $E$, and choose some $p \in \operatorname{Spec}(R)$ such that $p \in \operatorname{Ass}(M)$. Then $R / p$ embeds in $M$ and hence in $E$. Since $E_{R}(R / p)$ is essential over $R / p$ it follows that $E_{R}(R / p)$ also embeds in $E$, and therefore is a direct summand of $E$. As $E$ is indecomposable this shows that $E \cong E_{R}(R / p)$.

Now let $E$ be any injective $R$ module. The same argument as in the above paragraph shows that $E$ contains $E_{R}(R / p)$ for some $p \in \operatorname{Spec}(R)$; simply take any associated prime of any finitely generated submodule of $E$. Consider the set $\Lambda$ of all sets $\Gamma=\left\{E_{i}: i \in 1\right\}$ such that $E_{i}$ is an indecomposable injective submodule of $E$ and $\Sigma E_{i}=\oplus E_{i}$ in $E$. Ordering these sets by inclusion, we may apply Zorn's lemma to obtain a maximal such set $\Gamma=\left\{E_{i}: i \in 1\right\}$. Let $N=\Sigma E_{i} \subset E$. Then 
$N$ is injective since it is a direct sum of injectives and $R$ is Noetherian. Hence $E=N \oplus N^{\prime}$ for some submodule $N^{\prime}$ of $E$ which is necessarily injective. Then as above we may find some $L \cong E_{R}(R / p)$ with $L \subset N^{\prime}$. However then $\Gamma \cup\{L\}$ is strictly bigger than $\Gamma$ and lies in $\Lambda$. This contradiction finishes the proof of the theorem.

This theorem reduces the theory of injective modules over Noetherian ring to the study of the injective hulls of $R / p$ as $p$ ranges over the prime ideals of $R$. The theorem has an important consequence. Suppose that $x \in p$. Then $x$ acts nilpotently on $E_{R}(R / p)$ (as the only associated prime of $E_{R}(R / p)$ is $\left.p\right)$, so that $E_{R}(R / p)_{x}=0$. On the other hand, if $x \notin p$, then we know that $E_{R}(R / p)$ is divisible by $x$ since it is injective, and $x$ also acts injectively on this module since the only associated prime of $E_{R}(R / p)$ is $p$. This means that $x$ acts as a unit on this module, and hence $E_{R}(R / p)_{x}=E_{R}(R / p)$. In particular if $E$ is an arbitrary injective module over a Noetherian ring $R$, then $E$ maps onto $E_{x}$ and the latter module is injective. The kernel of this map is exactly $\Gamma_{x}(E)$. This observation yields a powerful inductive exact sequence on local cohomology:

TheOREM 3.2. Let $R$ be a Noetherian ring, and let $M$ be a finitely generated $R$-module. Fix an ideal $I$ in $R$ and an element $x \in R$. Set $J=(I, x)$. There is a long exact sequence

$$
0 \rightarrow H_{J}^{0}(M) \rightarrow H_{I}^{0}(M) \rightarrow H_{I}^{0}\left(M_{x}\right) \rightarrow H_{J}^{1}(M) \rightarrow H_{I}^{1}(M) \rightarrow H_{I}^{1}\left(M_{x}\right) \rightarrow \ldots
$$

Proof. Let $E^{\bullet}$ be an injective resolution of $M$. By the above remarks, there is an exact sequence of these resolutions,

$$
0 \rightarrow \Gamma_{x}\left(E^{\bullet}\right) \rightarrow E^{\bullet} \rightarrow E_{x}^{\bullet} \rightarrow 0
$$

This is a split short exact sequence of complexes, since each term in $E^{\bullet}$ is a direct sum of indecomposable injectives $E_{R}(R / p)$, and the two outer terms simply pick out whether $x \in p$ or $x \notin p$. Hence applying $\Gamma_{I}$ to this sequence maintains exactness. We then get a short exact sequence of complexes,

$$
0 \rightarrow \Gamma_{I}\left(\Gamma_{x}\left(E^{\bullet}\right)\right) \rightarrow \Gamma_{I}\left(E^{\bullet}\right) \rightarrow \Gamma_{I}\left(E_{x}^{\bullet}\right) \rightarrow 0
$$

whose associated long exact sequence proves the claim.

The above long exact sequence is closely related to another sequence which is the local cohomological version of the Mayer-Vietoris sequence. Fix ideals $I$ and $J$ in a Noetherian ring $R$. The ideals $\left\{I^{n}+J^{n}\right\}$ are cofinal with $\left\{(I+J)^{n}\right\}$ and $\left\{I^{n} \cap J^{n}\right\}$ are cofinal with $\left\{(I \cap J)^{n}\right\}$ (see Exercise 14). Applying $\operatorname{Hom}_{R}(, M)$ to the short exact sequences and taking direct limits,

$$
0 \rightarrow R /\left(I^{n} \cap J^{n}\right) \rightarrow R / I^{n} \oplus R / J^{n} \rightarrow R /\left(I^{n}+J^{n}\right) \rightarrow 0
$$

then gives a long exact sequence on local cohomology, which we refer to as the Mayer-Vietoris sequence:

$$
0 \rightarrow H_{I+J}^{0}(M) \rightarrow H_{I}^{0}(M) \oplus H_{J}^{0}(M) \rightarrow H_{I \cap J}^{0}(M) \rightarrow H_{I+J}^{1}(M) \rightarrow H_{I}^{1}(M) \oplus H_{J}^{1}(M) \rightarrow . .
$$

This sequence is called the Mayer-Vietoris sequence since it relates the cohomology of the complements of the closed sets $V(I)$ and $V(J)$ to the cohomology of the complement of $V(I) \cap V(J)$ and $V(I) \cup V(J)$.

ExAmple 3.3. A basic example of the Mayer-Vietoris sequence is provided by letting $R$ be a ring with an idempotent $e$, i.e., an element $e \in R$ such that $e^{2}=e$. In this case $V(e) \cup V(1-e)=$ $\operatorname{Spec}(R)=X$, and $V(e) \cap V(1-e)=\emptyset$. Hence these closed sets are open and give a disconnection of $X$. One would expect this to be realized in the 0th local cohomology of $R$. The disconnection should correspond to a direct summand of the local cohomology. Let $I=R e$ and $J=R(1-e)$. Then $I+J=R$, and $H_{R}^{i}(R)=0$ for all $i$, directly from the definition. Moreover, since $I \cap J$ and 
$I J$ have the same nilradical, and $I J=0$, we see that $H_{I \cap J}^{i}(R)=0$ for all $i>0$ and equals $R$ for $i=0$. The Mayer-Vietoris sequence then gives that $H_{I}^{0}(R) \oplus H_{J}^{0}(R)=R$ and $H_{I}^{i}(R)=0$ for all $i>0$. Moreover $H_{I}^{0}(R)=J$ and $H_{J}^{0}(R)=I$.

Of particular importance is the injective hull of the residue field $k$ of a local Noetherian ring $R$. We write $(R, \mathfrak{m}, k, E)$ to mean a Noetherian local $\operatorname{ring} R$ with maximal ideal $\mathfrak{m}$, residue field $k$, and $E=E_{R}(k)$. A crucial definition is:

Definition 3.4. The Matlis dual of an $R$-module $M$ is the module $M^{\vee}:=\operatorname{Hom}_{R}\left(M, E_{R}(k)\right)$.

We denote the completion of $R$ by $\widehat{R}$. In his thesis at the University of Chicago, Eben Matlis proved a fundamental duality result. See $[\mathbf{2 8}]$.

Theorem 3.5. (Matlis duality). Let $(R, \mathfrak{m}, k, E)$ be a Noetherian local ring.

(1) Any Artinian R-module $T$ is isomorphic to a submodule of $E^{r}$ for some integer $r$.

(2) There is a 1-1 correspondence between finitely generated $\widehat{R}$-modules and Artinian R-modules. This correspondence is given as follows: if $M$ is finitely generated then $M^{\vee}=\operatorname{Hom}_{\widehat{R}}(M, E)$ is Artinian. If $T$ is Artinian, then $T^{\vee}=\operatorname{Hom}_{R}(T, E)$ is finitely generated over $\widehat{R}$. Furthermore $N^{\vee \vee}=N$ if $N$ is a finitely generated module over $\widehat{R}$. Moreover, $E$ and $\widehat{R}$ are Matlis duals.

ExAmple 3.6. An important example of a Matlis dual is provided by a duality between Ext and Tor. Let $(R, \mathfrak{m}, k, E)$ be a local ring and suppose that $M$ and $N$ are $R$-modules. Then

$$
\operatorname{Tor}_{i}^{R}(M, N)^{\vee} \cong \operatorname{Ext}_{R}^{i}\left(M, N^{\vee}\right) \text {. }
$$

To prove this, let $\mathbb{F}$ be a free resolution of $M$. The module $\operatorname{Tor}_{i}^{R}(M, N)$ is computed as the homology of $\mathbb{F} \otimes_{R} N$. Dualizing into $E$ commutes with homology, so that $\operatorname{Tor}_{i}^{R}(M, N)^{\vee}$ is the homology of $\left(\mathbb{F} \otimes_{R} N\right)^{\vee} \cong \operatorname{Hom}_{R}\left(\mathbb{F}, N^{\vee}\right)$, where the last isomorphism is the Hom-tensor adjoint isomorphism. This latter homology is exactly $\operatorname{Ext}_{R}^{i}\left(M, N^{\vee}\right)$. This proof is valid with any injective module in place of $E$.

Moreover, if $M$ is finitely generated, then

$$
\operatorname{Ext}_{R}^{i}(M, N)^{\vee} \cong \operatorname{Tor}_{i}^{R}\left(M, N^{\vee}\right) .
$$

We leave the proof for the reader (see Exercise 12).

Before we can prove Matlis duality, more understanding about injective hulls is needed.

Proposition 3.7. Let $(R, \mathfrak{m}, k, E)$ be a local Noetherian ring. Every element of $E$ is annihilated by a power of $\mathfrak{m}$. Furthermore $E$ is a $\widehat{R}$-module, where $\widehat{R}$ is the completion of R. Moreover, $E \cong E_{\widehat{R}}(k)$.

Proof. If $x \in E$, then by Theorem 3.1, $\operatorname{Ass}(R x)=\{\mathfrak{m}\}$. It follows that some power of $\mathfrak{m}$ annihilates $x$. Let $\widehat{r} \in \widehat{R}$ and $x \in E$. Choose $n$ such that $\mathfrak{m}^{n} x=0$, and then choose an $r \in R$ such that $\widehat{r}-r \in \mathfrak{m}^{n}$. Define $\widehat{r} x=r x$. It is clear that this gives a well-defined $\widehat{R}$-module structure to $E$ which agrees with its $R$-module structure. Since $E$ is an essential extension of $k$ as an $R$-module, it necessarily is also an essential extension of $k$ as an $\widehat{R}$-module. Then $E \subset E^{\prime}=E_{\widehat{R}}(k)$ and $E^{\prime}$ is an $\widehat{R}$-module which is an essential extension of $k$ as an $\widehat{R}$-module. To show that $E$ is an injective hull of $k$ as an $\widehat{R}$-module it suffices to see that $E^{\prime}=E$, and to show this it is enough to show that $E^{\prime}$ is an essential extension of $k$ as an $R$-module, since $E$ is a maximal essential extension of $k$ as an 
$R$-module. Let $x \in E^{\prime}$, and choose an element $\widehat{r} \in \widehat{R}$ such that $\widehat{r} x \in k, \widehat{r} x \neq 0$. By the first part of this theorem, applied to $\widehat{R}, E^{\prime}$, and $x$, there is a power $n$ of $\widehat{\mathfrak{m}}$ such that $(\widehat{\mathfrak{m}})^{n} x=0$. Choose $r \in R$ such that $r-\widehat{r} \in(\widehat{\mathfrak{m}})^{n}$; then $\widehat{r} x=r x \in k$, and $r x \neq 0$. Hence $E^{\prime}$ is essential over $k$ as an $R$-module, and $E^{\prime}=E$.

To proceed further we need more specific information concerning the injective hull of the residue field in the case that $R$ is 0 -dimensional. The next result gives us what we will need. By $\lambda())$ we denote the length of an $R$-module, and by $M^{\vee}$ we will mean $\operatorname{Hom}_{R}(M, E)$. Recall that $\lambda(M)$ is the length of a filtration of $M$ by an ascending chain of submodules, $0=M_{0} \subseteq M_{1} \subseteq \ldots \subseteq M_{n}=M$ such that all quotient modules $M_{i+1} / M_{i}$ are isomorphic with $k$, the residue field of the local ring $R$. If no such finite filtration exists, then $M$ is said to be of infinite length. This concept is well defined, and is additive on short exact sequences. In particular, if $N \subseteq M$ and $\lambda(M)=\lambda(N)$ is finite, then $N=M$. A finitely generated $R$-module is Artinian if and only if it has finite length.

REMARK 3.8. Let $R \rightarrow S$ be a surjective local ring homomorphism of Noetherian local rings, and let $E$ be the injective hull of the residue field $k$ of $R$. Then $\operatorname{Hom}_{R}(S, E)$ is isomorphic with the injective hull of the residue field of $S$. This follows because by Hom-tensor adjointness this module is injective, but it is also essential over $k$. See Exercise 13 .

Proposition 3.9. Let $(R, \mathfrak{m}, k, E)$ be a 0-dimensional local Noetherian ring.

(1) $\lambda(M)=\lambda\left(M^{\vee}\right)$ for any finitely generated module $M$.

(2) $E^{\vee}=\operatorname{Hom}_{R}(E, E) \cong R$.

Proof. We show (1) by induction on $\lambda(M)$. If $\lambda(M)=1$, then $M \cong k$. But $k^{\vee}=\operatorname{Hom}_{R}(k, E)=$ $\operatorname{Hom}_{R}\left(k, E_{R}(k)\right) \cong E_{k}(k)$ by Remark 3.8. However $E_{k}(k)=k$, which shows that i) holds for $M=k$. Since $\operatorname{Hom}_{R}(, E)$ preserves short exact sequences (although it flips the arrows) it now easily follows by induction that i) is true for any module $M$ of finite length.

Since $R^{\vee}=E$, using part (1) twice we see that $\lambda(R)=\lambda\left(E^{\vee}\right)$. Hence to show $R \cong E^{\vee}$, it suffices to prove that the map induced by sending $r \in R$ to multiplication by $r$ on $E$ is injective. Suppose not; then there is some non-zero $r \in R$ with $r E=0$. Then $E \cong \operatorname{Hom}_{R}(R / R r, E) \cong E_{R / R r}(k)$ by Remark 3.8. By part (1), however, $\lambda\left(E_{R / R r}(k)\right)=\lambda(R / R r)<\lambda(R)$. This contradiction finishes the proof.

The next result generalizes the second part of the above proposition to arbitrary dimension and is a very important fact concerning the injective hull of the residue class field.

THEOREM 3.10. Let $(R, \mathfrak{m}, k, E)$ be a local Noetherian ring. Then $E^{\vee}=\operatorname{Hom}_{R}(E, E) \cong \widehat{R}$, the completion of $R$.

Proof. Set $E_{n}=\left\{x \in E: \mathfrak{m}^{n} x=0\right\}$. By Theorem 3.1, $\cup_{n} E_{n}=E$. On the other hand, $E_{n} \cong \operatorname{Hom}_{R}\left(R / \mathfrak{m}^{n}, E\right)=E_{R / \mathfrak{m}^{n}}(k)$. Let $R_{n}=R / \mathfrak{m}^{n}$. Suppose that $f \in E^{\vee}$. By $f_{n}$ denote the restriction of $f$ to $E_{n}$. Notice that $f_{n}\left(E_{n}\right) \subset E_{n}$, so that we can consider $f_{n} \in \operatorname{Hom}_{R}\left(E_{n}, E_{n}\right)$. Conversely given any family $\left\{g_{n}\right\}$ of homomorphisms from $E_{n}$ to $E_{n}$ such that $g_{n}$ is the restriction of $g_{m}$ to $E_{n}$ for $m \geq n$, we may define a map $g \in E^{\vee}$ by $g(x)=g_{n}(x)$ whenever $x \in E_{n}$. Thus

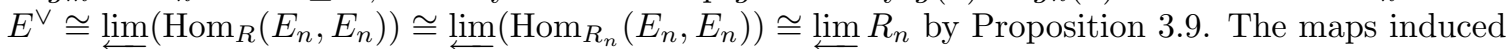
in the latter inverse limit are the natural surjections of $R_{m}$ onto $R_{n}$ for $m \geq n$ since the identity map restricts to the identity map. Thus $E^{\vee} \cong \lim _{n} R_{n} \cong \widehat{R}$.

REMARK 3.11. Let $(R, \mathfrak{m}, k, E)$ be a local Noetherian ring and let $M$ be an arbitrary $R$-module. If $M^{\vee}=0$, then $M=0$. 
Proof. First assume that $M$ is finitely generated. The composite of the maps

$$
M \longrightarrow M / \mathfrak{m} M \longrightarrow k \subset E,
$$

where the map from $M / \mathfrak{m} M$ to $k$ is just the projection onto some minimal generator, is a non-zero homomorphism in $M^{\vee}$. If $M$ is not finitely generated, take any finitely generated submodule $N$ of $M$, and a non-zero homomorphism of $N$ to $E$. Since $E$ is injective this extends to a non-zero homomorphism of $M$ to $E$.

Theorem 3.12. Let $(R, \mathfrak{m}, k, E)$ be a local Noetherian ring. Then $E$ is Artinian.

Proof. Let $\left\{M_{n}\right\}$ be a descending chain of submodules of $E$. Since $M_{n}$ is contained in $E, E^{\vee}=$ $\widehat{R}$ maps onto $\left(M_{n}\right)^{\vee}$. Let $I_{n}$ be the kernel of this surjection. Then $\left\{I_{n}\right\}$ is an ascending chain of ideals of $\widehat{R}$, and hence stabilizes. If $I_{n}=I_{n+1}=\ldots$, then $\left(M_{n}\right)^{\vee}=\left(M_{n+1}\right)^{\vee}=\ldots$ We will be done if we show that this implies that $M_{n}=M_{n+1}=\ldots$ It is enough to prove the next lemma.

LEMMA 3.13. Let $(R, \mathfrak{m}, k, E)$ be a local ring, and let $f: M \longrightarrow N$ be a homomorphism of $R$ modules. If $f^{\vee}: N^{\vee} \longrightarrow M^{\vee}$ is an isomorphism, then $f$ is an isomorphism.

Proof. Let $K=\operatorname{ker}(f)$. The exact sequence $0 \longrightarrow K \longrightarrow M \longrightarrow N$ becomes an exact sequence $N^{\vee} \longrightarrow M^{\vee} \longrightarrow K^{\vee} \longrightarrow 0$, after applying ()$^{\vee}$. Hence $K^{\vee}=0$ which by the remark above implies that $K=0$. Now let $C=\operatorname{coker}(f)$. Then there is a short exact sequence,

$$
0 \longrightarrow M \stackrel{f}{\longrightarrow} N \longrightarrow C \longrightarrow 0 \text {. }
$$

Applying $\vee$ to this sequence transposes it to a short exact sequence,

$$
0 \longrightarrow C^{\vee} \longrightarrow N^{\vee} \stackrel{f^{\vee}}{\longrightarrow} \rightarrow M^{\vee} \longrightarrow 0 .
$$

Hence $C^{\vee}=0$, and again by the remark, $C=0$ and $f$ is an isomorphism.

We can now prove the main result of this section, namely Matlis duality. This theorem gives a one-to-one arrow reversing correspondence between finitely generated modules over the completion of a Noetherian local ring $R$ and Artinian modules over $R$. The injective hull of the residue field plays the same role for Artinian modules as the completion of $R$ plays for finitely generated modules.

THEOREM 3.14. (Matlis Duality) Let $(R, \mathfrak{m}, k, E)$ be a Noetherian local ring.

(1) Any Artinian module $T$ is isomorphic to a submodule of $E^{r}$ for some integer $r$.

(2) There is a 1-1 correspondence between finitely generated $\widehat{R}$-modules and Artinian R-modules. This correspondence is given as follows: if $M$ is finitely generated then $M^{\vee}=\operatorname{Hom}_{\widehat{R}}(M, E)$ is Artinian. If $T$ is Artinian, then $T^{\vee}=\operatorname{Hom}_{R}(T, E)$ is finitely generated over $\widehat{R}$. Furthermore $N^{\vee \vee}=N$ if $N$ is a finitely generated module over $\widehat{R}$.

Proof. We first prove (1). Let $V=\operatorname{socle}(T)=\{x \in T: \mathfrak{m} x=0\}$. We claim that $T$ is an essential extension of $V$ and $V$ is finite dimensional. The second claim is clear since $V$ infinite dimensional implies that there exists an infinite descending chain of distinct subspaces of $V$ which necessarily are submodules of $T$, contradicting the fact that $T$ is Artinian. Let $x \in T$. Then $R x$ is also Artinian so that $\operatorname{Ass}(R x)=\{\mathfrak{m}\}$ and there is an $n$ such that $\mathfrak{m}^{n} x=0$ but $\mathfrak{m}^{n-1} x \neq 0$. Then $0 \neq \mathfrak{m}^{n-1} x \subset V \cap R x$. Hence $T$ is an essential extension of $V$. Set $r=\operatorname{dim}(V)$. Since $E_{R}(V)=E^{r}$ and the essential embedding of $V$ into $T$ extends to an embedding of $T$ into $E^{r}$, we have proved (1).

Suppose that $N$ is a finitely generated $\widehat{R}$-module. We can present $N:(\widehat{R})^{q} \longrightarrow(\widehat{R})^{p} \longrightarrow N \longrightarrow 0$, and after applying $\vee$ obtain that $N^{\vee} \subset(\widehat{R})^{\vee \oplus p}=\left(E_{\widehat{R}}(k)\right)^{p}=E^{p}$. Since $E$ is Artinian, so is $E^{p}$, and therefore so is $N^{\vee}$. Applying $\vee$ again yields the following commutative diagram: 


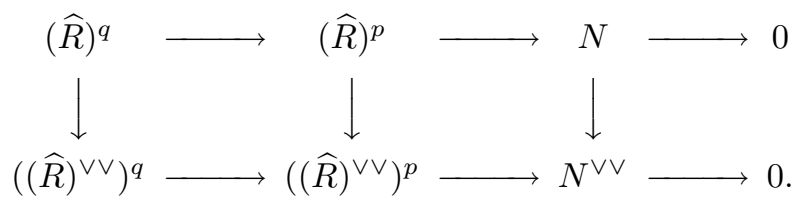

By Theorem 3.10, $(\widehat{R})^{\vee \vee} \cong \widehat{R}$ under the canonical map. The five-lemma then shows that $N \cong N^{\vee \vee}$.

Next assume that $T$ is an Artinian $R$-module. By the first part we can embed $T$ into $E^{r}$ for some $r$. The cokernel of this embedding is also Artinian so we can embed it into $E^{s}$ for some $s \geq 0$. Applying $\vee$ yields that $\left(E^{\vee}\right)^{r}$ maps onto $T^{\vee}$. Since $E^{\vee} \cong \widehat{R}$ by Theorem 3.10, it follows that $T^{\vee}$ is a finitely generated $\widehat{R}$-module. Applying ${ }^{\vee}$ twice gives a commutative diagram:

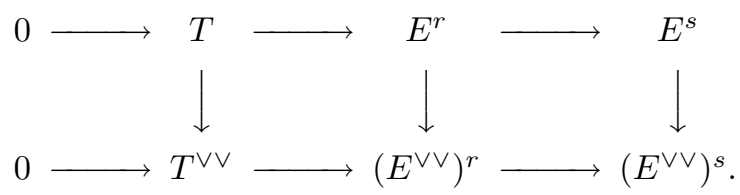

The five-lemma shows that $T \cong T^{\vee \vee}$ which finishes the proof.

\subsection{Exercises.}

EXERCISE 9. The argument in Example 3.3 shows that $H_{I}^{1}(R)=0$, with the notation as in that example. This cohomology is also computed from the Koszul cohomology as the cokernel of the map from $R \rightarrow R_{e}$. Explain directly why this map is surjective.

EXERCise 10. Let $R$ be a Noetherian ring, $I$ an ideal and $x \in R$. Prove for all $i \geq 2$, $H_{I \cap(x)}^{i}(R)=H_{I}^{i}\left(R_{x}\right)$. Explain how this connects the Mayer-Vietoris sequence associated to the ideals $I$ and $(x)$ to the long exact sequence of Theorem 3.2.

EXERCISE 11. Let $(R, \mathfrak{m})$ be a local Noetherian ring. The punctured spectrum of $R$ is the open set $U=\operatorname{Spec}(R) \backslash\{\mathfrak{m}\}$. Prove that $U$ is disconnected in the Zariski topology if and only if there exist two ideals $I$ and $J$, neither $\mathfrak{m}$-primary, such that $I+J$ is primary to $\mathfrak{m}$ and such that $I \cap J$ is nilpotent.

EXERCISE 12. Let $R$ be a Noetherian local ring and let $M$ be finitely generated. Prove

$$
\operatorname{Ext}_{R}^{i}(M, N)^{\vee} \cong \operatorname{Tor}_{i}^{R}\left(M, N^{\vee}\right) .
$$

EXERCISE 13. $S=R / I$ be a homomorphic image of a Noetherian local ring and let $M$ be an $S$ module. Prove that $\operatorname{Hom}_{S}\left(M, E_{S}(k)\right) \cong \operatorname{Hom}_{R}\left(M, E_{R}(k)\right)$. In particular $E_{S}(k) \cong \operatorname{Hom}_{R}\left(S, E_{R}(k)\right)$. (Hint: prove the latter statement first by showing that the module on the right hand side is injective and essential over $k$ as an $S$-module.)

Exercise 14. Let $R$ be a Noetherian ring, and let $I, J$ be ideal of $R$. Prove that $\left\{I^{n} \cap J^{n}\right\}$ is cofinal with $\left\{(I \cap J)^{n}\right\}$ and that $\left\{I^{n}+J^{n}\right\}$ is cofinal with $\left\{(I+J)^{n}\right\}$.

Exercise 15 . Prove that $E_{\mathbb{Z}}(\mathbb{Z} / p \mathbb{Z}) \cong \mathbb{Z}_{p} / \mathbb{Z}$. 


\section{Cohen-Macaulay and Gorenstein rings}

There are two types of Noetherian rings which are crucial in understanding and using local cohomology. These are Cohen-Macaulay and Gorenstein rings. Gorenstein rings are Cohen-Macaulay, and both properties are easily defined using local cohomology. We give a non-standard definition of the Gorenstein property which makes it easier to develop the material we need, especially the local duality theorem. The second appendix of this paper presents a more traditional development with full proofs. First, we need Cohen-Macaulay rings.

Definition 4.1. Let $R$ be a Noetherian ring, $I$ an ideal of $R$, and $M$ a finitely generated $R$-module such that $I M \neq M$. We define $\operatorname{depth}_{I}(M)$ to be the greatest integer $i$ such that for all $j<i, H_{I}^{j}(M)=0$. A local ring $(R, \mathfrak{m})$ is said to be Cohen-Macaulay (C-M for short) if $\operatorname{depth}_{\mathfrak{m}}(R)=\operatorname{dim}(R)$, i.e., if $H_{\mathfrak{m}}^{i}(R)=0$ for all $i<\operatorname{dim} R$.

Elements $x_{1}, \ldots, x_{n}$ in $R$ are said to be a regular sequence on $M$ if $\left(x_{1}, \ldots, x_{n}\right) M \neq M, x_{1}$ is not a zero divisor on $M$, and for all $2 \leq i \leq n, x_{i}$ is not a zero divisor on $M /\left(x_{1}, \ldots, x_{i-1}\right) M$. It is an easy exercise to prove that in the conditions of the definition, $\operatorname{depth}_{I}(M)$ is precisely the length of any (and hence all) maximal regular sequences in $I$ on $M$. We sketch a proof here, and leave the details as an exercise. First observe that $H_{I}^{0}(M)=0$ if and only if $I$ is not contained in any associated prime of $M$. Using prime avoidance this holds if and only if there exists an $x \in I$ which is a non-zerodivisor on $M$. This starts an induction. Applying the long exact sequence on local cohomology to the short exact sequence $0 \rightarrow M \stackrel{x}{\longrightarrow} M \rightarrow M / x M \rightarrow 0$ allows one to finish the proof, with the additional observation that an element $y \in I$ acts injectively on a local cohomology module $H_{I}^{j}(M)$ if and only if $H_{I}^{j}(M)=0$, because all elements of $I$ act nilpotently on such local cohomology.

A system of parameters in a local Noetherian ring $(R, \mathfrak{m})$ of dimension $d$ is a system of elements $x_{1}, \ldots, x_{d}$ such that $\sqrt{\left(x_{1}, \ldots, x_{d}\right)}=\mathfrak{m}$. One of the basic characterizations of dimension is exactly given by this definition: a system of parameters always exists, and $d$ is the least number of elements needed to generate $\mathfrak{m}$ up to radical. With this notation, $R$ is C-M if and only if every system of parameters form a regular sequence.

REMARK 4.2. Using the long exact sequence on local cohomology associated to the short exact sequence

$$
0 \rightarrow R \stackrel{x}{\rightarrow} R \rightarrow R / R x \rightarrow 0,
$$

where $x$ is a non-zerodivisor in a local Noetherian ring $R$, one can easily prove that $R$ is CohenMacaulay if and only if $R / R x$ is Cohen-Macaulay.

The next definition is a non-standard definition of a Gorenstein ring:

Definition 4.3. A local Noetherian ring of dimension $d,(R, \mathfrak{m}, k, E)$, is said to be Gorenstein if $R$ is Cohen-Macaulay and $H_{\mathfrak{m}}^{d}(R) \cong E$ (or equivalently, the Matlis dual of $H_{\mathfrak{m}}^{d}(R)$ is $\widehat{R}$ ).

The next theorem shows how good life is in a Gorenstein ring.

Theorem 4.4. (Local Duality) Let $(R, \mathfrak{m}, k, E)$ be a Gorenstein local ring of dimension d. Let $M$ be a finitely generated $R$-module. Then

(1) For $0 \leq i \leq d, H_{\mathfrak{m}}^{d-i}(M) \cong \operatorname{Ext}_{R}^{i}(M, R)^{\vee}$.

(2) If moreover $R$ is complete and $0 \leq i \leq d$, then $\left(H_{\mathfrak{m}}^{d-i}(M)\right)^{\vee} \cong \operatorname{Ext}_{R}^{i}(M, R)$.

In other words, $H_{\mathfrak{m}}^{d-i}(M)$ and $\operatorname{Ext}_{R}^{i}(M, R)$ are Matlis duals if $R$ is complete. 
Proof. Since $R$ is Cohen-Macaulay, $H_{\mathfrak{m}}^{i}(R)=0$ for $i<d$. Choose any system of parameters $x_{1}, \ldots, x_{d}$ for $R$. The complex $K^{\bullet}\left(x_{1}, \ldots, x_{d} ; R\right)$ is then a flat resolution of $H_{\mathfrak{m}}^{d}(R) \cong E$. By Remark 3.6, $\operatorname{Ext}_{R}^{i}(M, R)^{\vee} \cong \operatorname{Tor}_{i}^{R}(M, E)$, and we can compute this Tor by using the flat resolution $K^{\bullet}\left(x_{1}, \ldots, x_{d} ; R\right)$ of $E$; the homology in the $i$ th spot is the cohomology of $K^{\bullet}\left(x_{1}, \ldots, x_{d} ; R\right) \otimes_{R} M$ in the $(d-i)$ th spot, proving (1). The second claim follows from (1) by using Matlis duality.

The reason local duality is so important is that it allows the conversion of questions about local cohomology (especially their vanishing) to similar questions about Ext. This is very helpful because the Ext modules are finitely generated if $M$ is finitely generated, while local cohomology is seldom finitely generated. Another closely related reason can be seen by trying to localize local cohomology. Suppose one needs to study $H_{\mathfrak{m}}^{i}(M)$ for some finitely generated module $M$ over a Gorenstein local ring $R$ of dimension $d$. It may be important to use an inductive assumption by passing to the ring $R_{P}$ for some prime ideal $P \neq \mathfrak{m}$. But $\left(H_{\mathfrak{m}}^{i}(M)\right)_{P}=0$ since every element in $H_{\mathfrak{m}}^{i}(M)$ is annihilated by a power of $\mathfrak{m}$ ! So one apparently loses all information. But by local duality, $H_{\mathfrak{m}}^{i}(M)$ is the Matlis dual of $\operatorname{Ext}_{R}^{d-i}(M, R)$, and this module localizes quite nicely: $\operatorname{Ext}_{R}^{i}(M, R)_{P} \cong \operatorname{Ext}_{R_{P}}^{i}\left(M_{P}, R_{P}\right)$. Naturally, all of these useful properties would be fairly meaningless unless there are lots of Gorenstein rings. Luckily, there are.

Another important application of local duality is the fact that local cohomology with support in the maximal ideal is always Artinian.

Proposition 4.5. Let $(R, \mathfrak{m}, k, E)$ be a Noetherian local ring, and let $M$ be a finitely generated $R$-module. For all $i \geq 0, H_{\mathfrak{m}}^{i}(M)$ is Artinian.

Proof. By Proposition 2.15, we may assume that $R$ is complete. A basic fact we will use is a consequence of the Cohen Structure Theorem for complete local rings. We do not give the proof here, but simply state what we need:

Every complete local ring $R$ is a homomorphic image of a regular local ring $\left(A, \mathfrak{m}_{A}\right)$.

Choose $\left(A, \mathfrak{m}_{A}\right)$ a regular local ring of dimension $d$ mapping onto $R$. By the Independence of Base, $H_{\mathfrak{m}_{A}}^{i}(M)=H_{\mathfrak{m}}^{i}(M)$. By Local Duality,

$$
H_{\mathfrak{m}_{A}}^{i}(M) \cong\left(\operatorname{Ext}_{A}^{d-i}(M, A)\right)^{\vee},
$$

where $d=\operatorname{dim} A$. The module $\operatorname{Ext}_{A}^{d-i}(M, A)$ is finitely generated as an $A$-module, so that by Matlis Duality, $H_{\mathfrak{m}}^{i}(R)$ is Artinian as an $A$-module, and hence as an $R$-module.

A complete intersection is a local ring $R$ which after completion is the quotient of a regular local ring by a regular sequence. Of course, it suffices that this holds before completion. All such rings are Gorenstein. There is a heirarchy well-known to commutative algebraists: regular $\Longrightarrow$ complete intersection $\Longrightarrow$ Gorenstein $\Longrightarrow$ Cohen-Macaulay. In this article we will especially make use of complete intersections. In more concrete terms, a polynomial ring $k\left[x_{1}, \ldots, x_{n}\right]$ over a field $k$ is (locally) regular; a ring such as $k\left[x_{1}, \ldots, x_{n}\right] /\left(f_{1}, \ldots, f_{m}\right)$ is (locally) a complete intersection if $f_{1}, \ldots, f_{m}$ form a regular sequence, or equivalently if the height of $\left(f_{1}, \ldots, f_{m}\right)$ is $m$. The next proposition proves this fact.

Proposition 4.6. Let $R$ be a Noetherian local ring, and let $x_{1}, \ldots, x_{s}$ be a regular sequence in $R$. Then $R$ is Gorenstein if and only if $R /\left(x_{1}, \ldots, x_{s}\right)$ is a Gorenstein ring.

Proof. Assume that $R$ is Gorenstein. We proceed by induction on $i$ to prove that $R /\left(x_{1}, \ldots, x_{i}\right)$ is Gorenstein. It suffices to prove that if $(S, \mathfrak{m})$ is a Gorenstein local ring and $x$ is a non-zerodvisor in $S$, then $S / S x$ is Gorenstein. It is Cohen-Macaulay by Remark 4.2. The long exact sequence on local cohomology associated to the short exact sequence

$$
0 \rightarrow S \rightarrow S \rightarrow S / S x \rightarrow 0
$$


together with the facts that $H_{\mathfrak{m}}^{i}(S)=0$ for $i \neq d=\operatorname{dim}(S)$ and $H^{d}(S / x S)=0($ since $\operatorname{dim}(S / x S)=$ $d-1$ ) show that there is a short exact sequence,

$$
0 \rightarrow H_{\mathfrak{m}}^{d-1}(S / S x) \rightarrow H_{\mathfrak{m}}^{d}(S) \stackrel{x}{\rightarrow} H_{\mathfrak{m}}^{d}(S) \rightarrow 0
$$

If we dualize into the injective hull of the residue field of $S$, then since $S$ is Gorenstein, this identifies the Matlis dual of the highest local cohomology of $S$ with $\widehat{S}$, the completion of $S$, and identifies the Matlis dual of $H_{\mathrm{m}}^{d-1}(S / S x)$ with the completion of $S / S x$. Thus $S / S x$ is Gorenstein provided this dual is the Matlis dual of this module over the ring $S / S x$. We leave a more general statement as an exercise (Exercise 13) using Hom- $\otimes$ adjointness: if $S=R / I$ is a homomorphic image of a Noetherian local ring and $M$ is an $S$-module, then $\operatorname{Hom}_{S}\left(M, E_{S}(k)\right) \cong \operatorname{Hom}_{R}\left(M, E_{R}(k)\right)$. In particular $E_{S}(k) \cong \operatorname{Hom}_{R}\left(S, E_{R}(k)\right)$.

Next assume that $R /\left(x_{1}, \ldots, x_{s}\right)$ is Gorenstein. To prove that $R$ is Gorenstein, by induction it suffices to do the case in which $s=1$. Set $x=x_{1}$. Since $x$ is a non-zerodivisor and $R / x R$ is CohenMacaulay, it follows that $R$ is also Cohen-Macaulay. In particular, $H_{\mathfrak{m}}^{i}(R)=0$ for $i<d=\operatorname{dim} R$, and $H_{\mathfrak{m}}^{i}(R / x R)=0$ for $i<d-1$ (notice by the Independence of Base, it doesn't matter in the latter local cohomology whether we take the support to be the maximal ideal of $R$ or of $R / x R$ ). As above we get an exact sequence

$$
0 \rightarrow H_{\mathfrak{m}}^{d-1}(R / R x) \rightarrow H_{\mathfrak{m}}^{d}(R) \stackrel{x}{\rightarrow} H_{\mathfrak{m}}^{d}(R) \rightarrow 0 .
$$

Observe that $\operatorname{Hom}_{R}\left(H_{\mathfrak{m}}^{d-1}(R / R x), E\right)=\operatorname{Hom}_{R}\left(H_{\mathfrak{m}}^{d-1}(R / R x) \otimes_{R}(R / R x), E\right) \cong$ $\operatorname{Hom}_{R / R x}\left(H_{\mathfrak{m}}^{d-1}(R / R x), \operatorname{Hom}_{R}(R / R x, E)\right) \cong \operatorname{Hom}_{R / R x}\left(H_{\mathfrak{m}}^{d-1}(R / R x), E_{R / R x}(k)\right) \cong \widehat{R / R x}$, since by assumption $H_{\mathfrak{m}}^{d-1}(R / R x) \cong E_{R / R x}(k)$ and $\operatorname{Hom}_{R / R x}\left(E_{R / R x}(k), E_{R / R x}(k)\right) \cong \widehat{R / R x}$. Let $M=$ $\operatorname{Hom}_{R}\left(H_{\mathfrak{m}}^{d}(R), E\right)$, a finitely generated module over $\widehat{R}$. Taking the Matlis duals of the modules in the exact sequence above gives us an exact sequence,

$$
0 \rightarrow M \stackrel{x}{\longrightarrow} M \rightarrow \widehat{R / R x} \rightarrow 0
$$

To prove that $R$ is Gorenstein, we need to prove that $M \cong \widehat{R}$. Nakayama's lemma implies that $M$ is cyclic, hence can be written as $M \cong \widehat{R} / I$ for some ideal $I$. The exact sequence proves that $x$ is a non-zerodivisor on $I$, and moreover $I \subseteq \widehat{R} x$. Let $y \in I$, and write $y=x z$. Since $x$ is a non-zerodivisor on $\widehat{R} / I$, it follows that $z \in I$, and thus $I=x I$. Another application of Nakayama's lemma shows that $I=0$ and $M \cong \widehat{R}$. Therefore $R$ is Gorenstein.

Proposition 4.6 allows us construct new Gorenstein rings from old ones, but where do we start? We need a Gorenstein ring to begin with. Every regular local ring is Gorenstein, and applying Proposition 4.6 tells us that complete intersections are also Gorenstein. The proposition also tells us that to decide whether a Cohen-Macaulay local ring $R$ is Gorenstein, we can always simply decide whether or not $R /\left(x_{1}, \ldots, x_{d}\right)$ is Gorenstein, where $x_{1}, \ldots, x_{d}$ is a system of parameters. The ring $R /\left(x_{1}, \ldots, x_{d}\right)$ has a unique prime ideal which is maximal, and is Artinian. So the question becomes, when is an Artinian local ring Gorenstein?

Proposition 4.7. Let $(R, \mathfrak{m}, k, E)$ be an Artinian local ring containing its residue field $k$. Then $E$ is isomorphic to $\operatorname{Hom}_{k}(R, k)$.

Proof. It suffices to see that $\operatorname{Hom}_{k}(R, k)$ is both injective and essential over the residue field $k=$ $R / \mathfrak{m}$. To prove it is injective, we need to prove that $\operatorname{Hom}_{R}\left(\quad, \operatorname{Hom}_{k}(R, k)\right)$ is exact on short exact sequences of $R$-modules. By Hom-tensor adjointness, this functor is the same as $\operatorname{Hom}_{k}\left(\otimes_{R} R, k\right)$ which is certainly exact as $k$ is a field. To prove $\operatorname{Hom}_{k}(R, k)$ is essential over $k$, we first identify the copy of $k$ sitting inside this module. Map $R$ onto $k$ with kernel $\mathfrak{m}$, and apply $\operatorname{Hom}_{k}(, k)$. This 
embeds $\operatorname{Hom}_{k}(k, k)=k$ into $\operatorname{Hom}_{k}(R, k)$. Another way to say the same thing is that as a vector space, $R \cong k \oplus \mathfrak{m}$, with the copy of $k$ being generated by 1 . The generator of $\operatorname{Hom}_{k}(k, k)$ sending 1 to 1 takes $\mathfrak{m}$ to zero, so is just the projection of $R$ onto $k$. Let $N \subseteq \operatorname{Hom}_{k}(R, k)$ be an $R$-submodule of $\operatorname{Hom}_{k}(R, k)$. Choose any $f \in N$ which is nonzero. We need to prove there exists an element $r \in R$ such that $r f \in \operatorname{Hom}_{k}(k, k)$. Choose $n$ maximal such that there exists an element $r \in \mathfrak{m}^{n}$ with $f(r) \neq 0$. Consider $r f \in \operatorname{Hom}_{k}(R, k)$. We claim that $(r f)(\mathfrak{m})=0$ and $(r f)(1) \neq 0$. By definition, $(r f)(1)=f(r) \neq 0$. Moreover, if $z \in \mathfrak{m}$, then $(r f)(z)=f(r z)=0$ by construction. Therefore $r f \in \operatorname{Hom}_{k}(k, k)$, proving that $\operatorname{Hom}_{k}(R, k)$ is essential over $k$.

To study when an Artinian ring is Gorenstein, an important object to look at is the set of elements in the ring killed by the maximal ideal. This ideal has a name.

Definition 4.8. Let $(R, \mathfrak{m})$ be a local ring. The socle of $R$ is the ideal $0: \mathfrak{m}:=\{r \in R \mid r \mathfrak{m}=0\}$.

Let $R$ be a zero-dimensional graded ring, so that $R=k \oplus R_{1} \oplus R_{2} \oplus \ldots \oplus R_{s}$. When is $R$ Gorenstein? This is hard to answer in this generality. However, by the proposition above, note that the condition $R$ be Gorenstein is nothing but saying that $R=H_{\mathfrak{m}}^{0}(R) \cong E_{R}(k) \cong \operatorname{Hom}_{k}(R, k)$. Of course as vector spaces $R$ and $\operatorname{Hom}_{k}(R, k)$ are isomorphic, but the isomorphism needs to be as $R$-modules. A necessary condition is that the dimension of $R_{s}$ be exactly one. In fact, since $R$ is isomorphic to $E_{R}(k)$, the socle of $R$ must be one-dimensional. The copy of $k$ sitting inside of $E$ is clearly in the socle: if the dimension of the socle as a vector space were at least two, then one could choose a linear subspace of the socle of $E$ not intersecting the distinguished copy of $k$, contradicting the fact that $E$ is essential over $k$. Thus the dimension of the socle of $E$ is exactly one. In general, if $R$ is a 0 -dimensional graded ring as above, $R_{s}$ must sit in the socle since $R_{1} R_{s} \subset R_{s+1}=0$. For $R$ to be Gorenstein it is then necessary that $\operatorname{dim}_{k}\left(R_{s}\right)=1$, as claimed.

There are further restrictions on the Hilbert function of the a 0-dimensional Gorenstein ring $\mathrm{R}$, as $\operatorname{Hom}_{k}(R, k)$ turns the graded structure upside down. Even in the higher dimensional case, strong restrictions occur, summarized below. If $R$ is a standard graded ring, we define $H_{R}(t)=$ $\sum_{i=0}^{\infty} \operatorname{dim}_{k}\left(R_{n}\right) t^{n}$, the generating function for the Hilbert function of $R$. If $\operatorname{dim} R=d$, this power series can be written as a rational function:

$$
H_{R}(t)=\frac{h_{0}+h_{1} t+\ldots+h_{s} t^{s}}{(1-t)^{d}} .
$$

In the case in which $R$ is Artinian, $d=0$, and $h_{i}$ is simply the vector space dimension of $R_{i}$. The sequence $\left(h_{0}, \ldots, h_{s}\right)$ is called the h-vector of $R$. For example, an easy exercise shows that if $R=k\left[x_{1}, \ldots, x_{d}\right]$ is a polynomial ring, then $H_{R}(t)=\frac{1}{(1-t)^{d}}$. In the case $R$ is Gorenstein, this power series satisfies a basic functional equation:

THEOREM 4.9. Let $R$ be a standard graded algebra over $k$. Assume that $R$ is Gorenstein of dimension $d$. Then there exists an integer a such that

$$
t^{a} H_{R}\left(\frac{1}{t}\right)=(-1)^{d} H_{R}(t) .
$$

For example, we know that $R=k\left[x_{1}, \ldots, x_{d}\right]$ is Gorenstein. In this case $H_{R}\left(\frac{1}{t}\right)=\frac{t^{d}}{(t-1)^{d}}$, so that

$$
t^{-d} H_{R}\left(\frac{1}{t}\right)=(-1)^{d} H_{R}(t)
$$

One might hope that this functional equation characterizes Gorenstein rings, but this is not the case. For example, we can have 0-dimensional algebras with the same Hilbert function, e.g., $1, n, 1$, where one is Gorenstein and the other not Gorenstein. Explicitly $R=k[x, y] /\left(x y, x^{2}-y^{2}\right)$ is Gorenstein with $H_{R}(t)=1+2 t+t^{2}$. However, $S=k[x, y] /\left(x^{2}, x y, y^{3}\right)$ is not Gorenstein, but $H_{S}(t)=1+2 t+t^{2}$ as well. 
Although the equation above on the Hilbert series of a graded Gorenstein ring is not sufficient to guarantee the ring is Gorenstein, it is in many cases. The following theorem of Richard Stanley [37], 12.7 seems surprising.

THEOREM 4.10. Let $R$ be a standard graded domain over a field $k$. If $R$ is Cohen-Macaulay and has dimension $d$, then $R$ is Gorenstein if and only if there exists an integer a such that

$$
t^{a} H_{R}\left(\frac{1}{t}\right)=(-1)^{d} H_{r}(t) .
$$

See Exercise 2.11 for an example of this.

EXAMPLE 4.11. In the case $s=2$ above, it is not difficult to give a condition for Gorensteiness. We consider $R=k \oplus V \oplus k$, where $V=R_{1}$, and the second copy of $k$ sits in degree 2 . To specify a commutative ring structure on such a vector space, we need only say how to multiply two elements of degree 1. For, everything of degree at least 3 is zero, and multiplication by the copy of $k$ in degree 0 is just the usual vector space structure on each graded component. The multiplication of two degree one elements must land in the copy of $k$ in degree 2, thus giving a symmetric bilinear form $<, \quad>$ determined by multiplication. Let $V$ be a $n$-dimensional vector space over $k$. Recall that a symmetric bilinear form on $V$ is a pairing $V \times V \rightarrow k$ given by $(v, w) \mapsto<v, w>\in k$ such that $<,>$ is linear in each variable and is symmetric, i.e. $\langle v, w\rangle=\langle w, v\rangle$ for every $v, w \in V$.

Conversely given any symmetric bilinear form on a finite dimensional $k$-vector space $V$, we can make a commutative $\operatorname{ring} R=k \oplus V \oplus k$ by defining the multiplication of two elements $u, v \in V$ to be $\langle u, v\rangle$ in the degree two copy of $k$. A natural question is to ask when such a ring is Gorenstein in terms of the symmetric bilinear form. There is a nice answer.

Note that $R \simeq k\left[X_{1}, \ldots, X_{n}\right] / I$, where $I$ is a homogeneous ideal such that $\mathfrak{m}^{3} \subseteq I$. Now $R$ is Gorenstein if and only if $0::_{R} \mathfrak{m}=\operatorname{socle}(R)$ is a one-dimensional $k$-vector space. Since $\mathfrak{m}^{3}=0$, we see that $\mathfrak{m}^{2} \subseteq \operatorname{socle}(R)$. Hence $R$ is Gorenstein if and only if $\mathfrak{m}^{2}=\operatorname{socle}(R)$. In other words, $R$ is Gorenstein if and only if $R_{1} \cap \operatorname{socle}(R)=0$, i.e. $\operatorname{socle}(R)$ does not contain any linear forms.

For a subspace, $W \subseteq V$, we define

$$
W^{\perp}=\{v \in V:<w, v>=0 \text { for every } w \in W\} .
$$

We say that the form $<,>$ is non-degenerate if $V^{\perp}=0$. The condition that $R_{1} \cap \operatorname{socle}(R)=0$, i.e. that $R$ be Gorenstein, can now be rephrased:

$R$ is Gorenstein if and only if $<,>$ is a non-degenerate symmetric bilinear form on $R_{1}$.

Let $\left\{v_{1}, \ldots, v_{n}\right\}$ be a $k$-basis for $V$. Given a symmetric bilinear form $<,>$ on $\mathrm{V}$, we can associate to it a symmetric matrix as follows:

$$
<,>\longleftrightarrow\left(<v_{i}, v_{j}>\right) .
$$

One can check that $<,>$ is non-generate if and only if the matrix $\left.\left(<v_{i}, v_{j}\right\rangle\right)$ is invertible.

For a particular example, consider the case in which the Hilbert function of $R$ is $1,3,1$. Let $V$ be a 3 -dimensional vector space over a field $k$. The identity matrix

$$
\left(\begin{array}{lll}
1 & 0 & 0 \\
0 & 1 & 0 \\
0 & 0 & 1
\end{array}\right)
$$

corresponds to a non-degenerate symmetric bilinear form on $V$. The corresponding ring $R=k \oplus$ $R_{1} \oplus R_{2}$ is Gorenstein, where $R_{1}=k \cdot x \oplus k \cdot y \oplus k \cdot z, R_{2}=k \Delta$ satisfying $x^{2}=y^{2}=z^{2}=\Delta, x y=0$, $y z=0$ and $x z=0$, i.e. $R \simeq k[X, Y, Z] /\left(X^{2}-Y^{2}, X^{2}-Z^{2}, X Y, Y Z, X Z\right)$ is Gorenstein.

REMARK 4.12. Gorenstein rings with Hilbert function 1, n, n, 1 have not been classified. 
In fact there are many fascinating questions concerning 0-dimensional graded Gorenstein rings. The Charney-Davis conjecture ([10]) from combinatorics is one. The treatment below is taken from [34]; I thank Vic Reiner for explaining the conjecture to me. Let $\Delta$ be an abstract simplicial complex triangulating a $(d-1)$-dimensional homology sphere. The $\mathrm{f}$-vector is the list $\left(f_{-1}, f_{0}, \ldots, f_{d-1}\right)$, where $f_{i}$ is the number of $i$-dimensional faces. Write $f(t)=\sum_{i=0}^{d} f_{i-1} t^{i}$, and define $h_{i}$ by the functional equation $t^{d} h(t)=\left[t^{d} f\left(t^{-1}\right)\right]_{t \rightarrow t-1}$. The Charney-Davis conjecture states that if $\Delta$ is a flag simplicial homology sphere and $d$ is even, then

$$
(-1)^{\frac{d}{2}} h(-1) \geq 0 .
$$

The complex $\Delta$ is said to be a flag complex if the minimal subsets of vertices which do not span a simplex all have cardinality two.

We can translate this conjecture into commutative algebra using the Stanley-Reisner ideal associated with $\Delta, I=I_{\Delta}$. The ideal $I$ is defined to be the ideal generated by square-free monomials $x_{j_{1}} x_{j_{2}} \cdots x_{j_{r}}$ such that $\left\{j_{1}, \ldots, j_{r}\right\}$ is not a face in $\Delta$. Homology spheres are Cohen-Macaulay, so that $S / I$ is Cohen-Macaulay, where $S$ is the polynomial ring $k\left[x_{1}, \ldots, x_{n}\right]$ on the vertices of $\Delta$, over a field $k$. Moreover $S / I$ is even Gorenstein. The assumption that $\Delta$ is a flag complex means that $I$ is generated by quadrics. We can cut down by general linear forms (if $k$ is infinite) to reach a 0 -dimensional Gorenstein ring defined by quadrics. In this case $h(t)$ is simply the Hilbert series for this ring. We can now pose the Charney-Davis conjecture as a more general question:

Question: Let $R$ be a graded zero-dimensional Gorenstein ring defined by quadrics, and let $h_{i}=$ $\operatorname{dim}_{k} R_{i}$. Suppose that $h_{d} \neq 0, h_{d+1}=0$, and $d$ is even. Is

$$
(-1)^{\frac{d}{2}} h(-1) \geq 0 \text { ? }
$$

This question is open in this generality even for $d=4$. In this case the Hilbert series is of the form $1, n, m, n, 1$, and the question asks whether $m \geq 2 n-2$ ?

\subsection{Exercises.}

EXERCISE 16. Use the Mayer-Vietoris sequence to prove the following well-known result of Hartshorne: if $(R, \mathfrak{m})$ is a local Noetherian ring having depth at least two, then the punctured spectrum, $\operatorname{Spec}(R) \backslash\{\mathfrak{m}\}$ is connected.

EXERCISE 17. Justify the assertion that a regular local ring is a Gorenstein ring in the case in which $R=k\left[x_{1}, \ldots, x_{n}\right]$, by using the explicit isomorphism of Exercise $6, H_{\mathfrak{m}}^{n}(R) \cong k\left[x_{1}^{-1}, \ldots, x_{n}^{-1}\right]$, to prove that $H_{\mathfrak{m}}^{n}(R)$ is an injective hull of the residue field of $R$ (as graded ring).

EXERCISE 18. Let $x$ be a non-zerodivisor in a local Noetherian ring $R$. Directly from Definition 4.1, prove that $R$ is Cohen-Macaulay if and only if $R / R x$ is Cohen-Macaulay.

EXERCISE 19. Let $R=k[x u, x v, y u, y v]$ as a subring of the polynomial ring $k[x, y, u, v]$. This ring is a Cohen-Macaulay domain. Given this, prove it is Gorenstein using Stanley's theorem by showing that the Hilbert series of $R$ is $H_{R}(t)=\frac{t+1}{(1-t)^{d}}$.

EXERCISE 20. Let $(R, \mathfrak{m})$ be a Noetherian local ring, I a proper ideal, and $M$ a finitely generated $R$-module. Set $t=\operatorname{depth}_{I} M$. Prove that $t$ is the length of the longest regular sequence $x_{1}, \ldots, x_{t} \in I$ on $M$. 


\section{Vanishing Theorems and the Structure of $H_{\mathfrak{m}}^{d}(R)$}

In this section we will discuss vanishing theorems for local cohomology. The cohomological dimension of $I$ in $R$, denoted by $c d(R, I)$, is the smallest integer $n$ such that the local cohomology modules $H_{I}^{q}(M)=0$ for all $R$-modules $M$, and for all $q>n$. It is enough to take $M=R$ in this definition (Exercise 21).

There are three basic vanishing results. The first is an immediate consequence of the identification of Koszul cohomology with local cohomology, but is important enough to warrant its own theorem, Theorem 5.4.

Definition 5.1. Let I be an ideal in a commutative Noetherian ring. We set ara(I) equal to the least integer $n$ such that I can be generated by $n$ equations up to radical.

The Krull height theorem (see Theorem 1.1) proves that in any Noetherian ring, $\operatorname{ara}(I) \geq \mathrm{ht}(I)$.

EXAmple 5.2. Let $k$ be a field, and set $R=k[x, y, z] /\left(x^{2}-y z\right) . R$ is a two-dimensional ring with a height one prime $p=(x, y)$ generated by two elements. However, $\operatorname{ara}(p)=1$ since $y$ generates $p$ up to radical.

ExAmPLE 5.3. For a less trivial example, consider the kernel $p$ of the surjective homomorphism of $k[x, y, z]$ onto $k\left[t^{3}, t^{4}, t^{5}\right]$ sending $x, y, z$ to $t^{3}, t^{4}, t^{5}$ respectively. The prime $p$ is height two (since $\left.\operatorname{dim} k\left[t^{3}, t^{4}, t^{5}\right]=1\right)$, and is minimally generated by the three equations $x^{3}-y z, y^{2}-x z, z^{2}-x^{2} y$, which are the $2 \times 2$ minors of the matrix

$$
A=\left(\begin{array}{ccc}
x & y & z \\
y & z & x^{2}
\end{array}\right) .
$$

Consider the $3 \times 3$ matrix obtained from $A$ by filling $A$ out to a symmetric matrix:

$$
B=\left(\begin{array}{ccc}
x & y & z \\
y & z & x^{2} \\
z & x^{2} & 0
\end{array}\right)
$$

Let $\Delta$ be the determinant of this matrix. Up to radical, $p$ is generated by $\Delta$ and $y^{2}-x z$. Hence $\operatorname{ara}(p) \leq 2$. But, since the height of $p$ is two, two is the minimal possible value.

A more general result holds. If $A$ is an $n$ by $n+1$ matrix whose first $n$ columns give an $n$ by $n$ symmetric matrix $C$, then concatenating $A$ and its transpose, and filling out this $n+1$ by $n+1$ matrix with a 0 to make a matrix $B$ gives that $\operatorname{det}(B)$ and $\operatorname{det}(C)$ generate up to radical the ideal generated by all of the maximal minors of $A$ (Exercise 22).

Theorem 5.4. Let $R$ be a commutative Noetherian ring and let $I$ be an ideal of $R$. Then $H_{I}^{i}(M)=0$ for all $i>\operatorname{ara}(I)$ and for all $R$-modules $M$.

Proof. This follows at once from the fact that we may compute the local cohomology by choosing $\operatorname{ara}(I)$ elements which generate $I$ up to radical and use the Koszul cohomology on these elements to calculate the local cohomology.

This theorem gives a lower bound for ara $(I)$. We give two famous examples of this criterion. The first example, due to Hartshorne, shows that two skew lines in space cannot be defined by two equations up to radical.

EXAmPle 5.5. (Two skew lines in $\left.\mathbb{P}^{3}\right)$ Let $k$ be a field, and set $R=k[x, y, u, v], I=(x, y) \cap(u, v)$. This ideal has height two. It is generated by the four elements $x u, x v, y u, y v$, and can be generated up to radical by the three elements $x u, y v, x v+y u$. Can it be defined up to radical by two equations? 
We use the Mayer-Vietoris sequence to prove it cannot be generated by two elements up to radical. A part of the Mayer-Vietoris sequence is:

$$
H_{(x, y)}^{3}(R) \oplus H_{(u, v)}^{3}(R) \rightarrow H_{I}^{3}(R) \rightarrow H_{\mathfrak{m}}^{4}(R) \rightarrow \ldots
$$

where $\mathfrak{m}=(x, y)+(u, v)$. The two terms on the left-hand side of this displayed sequence are zero since they are taking the third local cohomology of ideals generated by two elements. The last term is nonzero by Theorem 5.9 below. Hence $H_{I}^{3}(R) \neq 0$, which gives that $\operatorname{ara}(I)>2$, by Theorem 5.4

ExAmple 5.6. $R=\mathbb{C}\left[X_{i j}\right], 1 \leq i \leq 2$ and $1 \leq j \leq 3$. Let $I$ be the ideal generated by the $2 \times 2$ minors of the $2 \times 3$ matrix $X$ given by entries $X_{i j}$. This ideal has 3 generators. Can it be generated up to radical by 2 elements? If so, the above theorem shows that $H_{I}^{3}(R)=0$, However, it is known that this module is nonzero. Hence $I$ cannot be generated up to radical by two elements. On the other hand in positive characteristic $H_{I}^{3}(R)=0$. It has been shown using étale cohomology that even in characteristic $p$ the ideal cannot be generated up to radical by two elements.

There are several proofs that $H_{I}^{3}(R)$ is not zero. One such proof uses the analytic topology of the complement of $I$, found in [18]. First, we state some topological information provided by the vanishing of local cohomology.

Let $R=\mathbb{C}\left[x_{1}, \ldots, x_{n}\right]$ be the polynomial ring in $n$-variables over the complex numbers, and let $I$ be an ideal of $R$. The following proposition connects the vanishing of local cohomology with the singular cohomology ${ }^{3}$ as an analytic space.

Proposition 5.7. Let $R=\mathbb{C}\left[x_{1}, \ldots, x_{n}\right]$ be the polynomial ring in $n$-variables over the complex numbers, and let $I$ be an ideal of $R$. Set $X=\operatorname{Spec}(R) \backslash V(I)$. If $H_{I}^{i}(R)=0$ for all $i>r$, then $H_{\text {sing }}^{i}(X ; \mathbb{C})=0$ for all $i>n+r-1$.

The proof of this proposition is beyond the scope of these notes, passing through an identification of the singular cohomology of $X$ with algebraic DeRham cohomology. This identification can be used to prove that $H_{I}^{3}(R) \neq 0 .{ }^{4}$

THEOREM 5.8. Let $R$ be a commutative Noetherian ring and let $I$ be an ideal and $M$ a finitely generated $R$-module. Then $H_{I}^{i}(M)=0$ for $i<\operatorname{depth}_{I}(M)$, and for $i>\operatorname{dim}(M)$.

\footnotetext{
${ }^{3}$ Recall the definition of the singular cohomology of a topological space with coefficients in a group $A$ : let $X$ be a topological space, and let $\Delta^{q}$ denote the standard $q$-simplex $\left\langle p_{0}, \ldots, p_{q}\right\rangle$ whose vertices $p_{i}$ are the unit coordinate vectors in $\mathbb{R}^{q+1}$. A singular $q$-simplex in $X$ is a continuous map $\sigma: \Delta^{q} \rightarrow X$. We let $C_{q}(X)$ be the free $A$-module generated by all singular $q$-simplices. There is a linear map $e_{q}^{i}: \Delta_{q-1} \rightarrow \Delta_{q}$ sending $\Delta_{q-1}$ to the face of $\Delta_{q}$ opposite $p_{i}$. The ith face $\sigma^{(i)}$ of $\sigma$ is the singular $(q-1)$-simplex which is the composite $\sigma^{(i)}:=\sigma e_{q}^{i}: \Delta_{q-1} \rightarrow \Delta_{q} \rightarrow X$. We define a linear homomorphism $\delta_{q}(\sigma)=\sum_{i=0}^{q}(-1)^{i} \sigma^{(i)}$ from $C_{q}$ to $C_{q-1}$. This is the singular chain complex of $X$ over $A$, where we give an augmentation map to $A$. The singular cohomology groups $H^{*}(X ; A)$ are the graded cohomology groups of the cochain complex $\operatorname{Hom}(C(X), A)$, augmented by $A$.

${ }^{4}$ The following argument is found in $[\mathbf{1 8}]$. Let $X=\operatorname{Spec}(R) \backslash V(I)$, where $I$ is the ideal generated by the 2 by 2 minors of a generic 2 by 3 matrix. $X$ consists of 2 by 3 matrices over the complex numbers of rank 2 . We think of these over the real numbers. It suffices to prove that $X$ is homotopic equivalent to a compact orientable manifold of real dimension 8 ; in this case $H^{8}(X ; \mathbb{C})$ is non-zero by Poincaré duality which says that for a compact connected oriented $n$-manifold $X, H_{q}(X ; A) \cong H^{n-q}(X ; A)$, where $A$ is a group of coefficients. By Proposition 5.7 , it follows that $H_{I}^{3}(R)$ is non-zero. Otherwise, setting $r=2$ in that discussion, with $n=6$, we would have that the vanishing of $H_{I}^{j}(R)$ for $j>2$ (note that all local cohomology from the fourth local cohomology on must then vanish since $I$ is defined by three elements) forces the vanishing of $H^{j}(X ; \mathbb{C})$ for $j>n+1=7$.

We can continuously change the first row of the matrix so that it has unit length. Then by continuously subtracting multiplies of the first row from the second we can assume they are orthogonal, and then repeating the first process, assume that the second row also has unit length. The first row is then a copy of the real five sphere $S^{5}$, and after fixing the first row, the second row varies in a three sphere $S^{3}$. Thus the total space is an $S^{3}$ bundle over $S^{5}$, which is simply connected. This proves that $X$ is homotopic equivalent to an 8-dimensional orientable compact manifold, as required.
} 
ProOF. The first statement is just a restatement of the definition of depth. To prove the second statement, we may use a prime filtration of $M$ to replace $M$ by $R / p$ for a prime $p$. We can then use the Independence of Base to replace $R$ by $R / p$ and $I$ by its image in $R / p$. Finally, it suffices to prove vanishing locally; as local cohomology commutes with localization, we can assume that $R$ is local. This reduces the second claim to proving that if $(R, \mathfrak{m}, k, E)$ is a local Noetherian domain of dimension $d$, then $H_{I}^{j}(R)=0$ for all $j>d$.

Since $\operatorname{ara}(I) \leq d$, our first Theorem immediately gives this claim. However, since this may not be well-known to all readers, we give an alternate proof using the Mayer-Vietoris type sequence of the first section. We induct upon the dimension of $R$ and a second induction on the dimension of $R / I$. If $\operatorname{dim}(R / I)=0$, then $\sqrt{I}=\mathfrak{m}$, and by definition of dimension, ara $(\mathfrak{m})=d$, proving the claim. If $\operatorname{dim}(R / I)>0$, choose $x \in \mathfrak{m}, x$ not in any minimal prime of $I$. Then $\operatorname{dim}(R /(I, x))<\operatorname{dim}(R / I)$. But for $j>\operatorname{dim}\left(R_{x}\right)$ our induction proves that $H_{I}^{j}\left(R_{x}\right)=0$, and then the Mayer-Vietoris sequence shows that $H_{(I, x)}^{j}(R)$ maps onto $H_{I}^{j}(R)$, and the second induction finishes the proof.

TheOREM 5.9. Let $(R, \mathfrak{m}, k, E)$ be a local Noetherian ring of dimension $d$.

(1) For every $i, H_{\mathfrak{m}}^{i}(R)$ is an Artinian module.

(2) $H_{\mathfrak{m}}^{d}(R) \neq 0$. More generally, for a finitely generated $R$-module $M$ of dimension $t, H_{\mathfrak{m}}^{t}(M) \neq 0$.

Proof. Part (1) is a special case of Proposition 4.5. For all parts we may complete $R$ without loss of generality. Henceforth we assume that $R$ is complete.

The proof of (2) follows from Local Duality. Map a regular local $\operatorname{ring}\left(A, \mathfrak{m}_{A}\right)$ onto $R$ using the Cohen Structure Theorem, so that we can write $R=A / I$ for some ideal $I$ of height $h$. Choose a maximal regular sequence $x_{1}, \ldots, x_{h} \in I$, and set $B=A /\left(x_{1}, \ldots, x_{h}\right)$. Then $B$ is a complete intersection, hence Gorenstein, $B$ maps onto $R$, and the dimension of $B$ is the same as the dimension of $R, d$. Local duality shows that $H_{\mathfrak{m}}^{d}(R)=H_{\mathfrak{m}_{B}}^{d}(R)=\left(\operatorname{Ext}_{B}^{0}(R, B)\right)^{\vee}=\operatorname{Hom}_{B}(R, B)$. But $\operatorname{Hom}_{B}(R, B)$ is nonzero since $R=B / J$ where $J=I /\left(x_{1}, \ldots, x_{h}\right)$ has height 0 , and $\operatorname{Hom}_{B}(R, B)$ can be identified with $0:_{B} I$, which is nonzero.

The more general claim for a module $M$ follows similarly. Set $J=0:_{R} M$, the annihilator of $M$. Choose $B$ as in the above paragraph. Furthermore choose a maximal regular sequence $z_{1}, \ldots, z_{h}$ in $J$, and set $C=B /\left(z_{1}, \ldots, z_{h}\right)$. Local duality gives that $H_{\mathfrak{m}}^{t}(M)=H_{\mathfrak{m}_{C}}^{t}(M)=\left(\operatorname{Ext}_{C}^{\operatorname{dim} C-t}(M, C)\right)^{\vee}$. However, $\operatorname{dim} C-t=\operatorname{dim} B-h-t=\operatorname{dim} R-h-t=0$, since $t=\operatorname{dim} M=\operatorname{dim} R-h$. It then suffices to prove that $\operatorname{Hom}_{C}(M, C) \neq 0$. Choose any minimal prime $P$ of $J$ having maximal dimension. It is enough to prove that $\left.\operatorname{Hom}_{C}(M, C)\right)_{P} \neq 0$. In this case, $C_{P}$ is a 0 -dimensional Gorenstein ring, and $M_{P} \neq 0$, so there is a nonzero homomorphism from $M_{P}$ to $C_{P}$ by Matlis duality, for example.

The last two subsections of this section give some additional information, without proofs, concerning the structure of local cohomology. They can be skipped if the reader prefers; they will not be used in the rest of this article.

5.1. The Graded Case Revisited. Let $R=\oplus_{i \geq 0} R_{i}$ be a graded Noetherian ring over a field $k=R_{0}$. Set $\mathfrak{m}$ equal to the ideal generated by all positive degree elements. This ideal is called the irrelevant ideal. But it is far from irrelevant! Exactly the same proof as above shows that $H_{\mathfrak{m}}^{d}(R)$ is Artinian (For the ring $A$, use a polynomial ring mapping onto $R$ factored by a maximal regular sequence in the annihilator of $R$ as a module over the polynomial ring.) Since $H_{\mathfrak{m}}^{d}(R)$ is Artinian, there must be a maximal degree in which there is a nonzero element. One can see this claim by considering the descending chain of submodules $M_{i}$ generated of all elements of degree at least $i$. It must stabilize for some large $i$, but this then forces these stable submodules to be zero. This highest degree is called the a-invariant of $R$, and is an extremely important invariant of the ring. 
In the calculation we did earlier, we can now say that the a-invariant of a polynomial ring $k[X]$ is -1 , while the a-invariant of $k[X, Y]$ is -2 . It is not hard to guess that the a-invariant of a polynomial ring in $n$-variables is $-n$. But don't be misled by this negativity. When the a-invariant is negative, the ring is good in many ways. In some sense most rings have positive a-invariant. For example if $R=k\left[x_{1}, \ldots, x_{n}\right] /(F)$ is a hypersurface where $F$ has degree $d$, then the a-invariant of $R$ is exactly $d-n$ (Exercise 23). An example of the good behavior of rings with negative a-invariant is provided by a result of Buchweitz. To state it, recall that the Hilbert function of a Noetherian graded ring $R=\oplus R_{i}$ over a field $R_{0}=k$ is the function sending $n$ to the vector space dimension of $R_{n}$. This function is a polynomial in $n$ for large $n$, having degree one less than the Krull dimension of $R$, called the Hilbert polynomial of $R$.

Proposition 5.10. Let $R=R_{0}\left[R_{1}\right]$ be a standard graded ring over a field $k=R_{0}$. Assume that $R$ is Cohen-Macaulay and $a(R)<0$. Then the Hilbert polynomial and the Hilbert function take the same values for all $n \geq 0$.

We leave the proof as an exercise (Exercise 30).

One of the most basic and important vanishing theorems in algebraic geometry is the Kodaira vanishing theorem. A consequence of it is the following statement about the vanishing of graded pieces of local cohomology. (see [21]). The proof is beyond the scope of these notes.

Theorem 5.11. (A Variation of Kodaira Vanishing Theorem) Let $S$ be a standard graded algebra over the complex numbers with irrelevant ideal $\mathfrak{m}$, and assume that $S$ is an integrally closed domain such that $S_{P}$ is regular for every prime $P$ not equal to the irrelevant ideal $\mathfrak{m}$. For all $i<\operatorname{dim}(S)$, and for all $j<0$,

$$
\left[H_{\mathfrak{m}}^{i}(S)\right]_{j}=0 .
$$

5.2. More Structure: Local Cohomology as a $D$-module. We follow the treatment of Lyubeznik [26]. Let $R=k\left[x_{1}, \ldots, x_{n}\right]$ or $R=k\left[\left[x_{1}, \ldots, x_{n}\right]\right]$ be a polynomial ring or power series ring over a base ring $k$. Define differential operators $D_{i, t}=\frac{1}{t !} \frac{\delta^{t}}{\delta x_{i}^{t}}$, the partial differentation of order $t$ with respect to $x_{i}$. This formal expression does give an honest operator even if $t$ ! is not invertible, since differentiation yields a coefficient divisible over the integers by $t$ !, and this integer coefficient is what we mean by dividing by $t$ !. These operators live inside $\operatorname{Hom}_{k}(R, R)$, as does multiplication by elements of $R$. The subring generated by these operators and by multiplication by elements of $R$ we call $D$. $D$ turns out to be the ring of all $k$-differential operators of $R$, though we do not need this fact here. ${ }^{5}$ Localizations of $R$ are also $D$-modules by extending the action using the quotient rule. Finally, using the Koszul cohomology to compute local cohomology shows that for all $i, H_{I}^{i}(R)$ are also $D$-modules. What is amazing is that as $D$-modules, these local cohomolgy modules are finitely generated, though they are usually non-finitely generated as $R$-modules. Lyubeznik $[\mathbf{2 5}],[\mathbf{2 6}]$ has used this point of view to prove many results concerning local cohomology, and it has been used by Uli Walther [40] and others to give algorithms for the computation of local cohomology.

A good case to consider is the $D$-module $R_{f}$, where $R$ is as above. The fact that this $D$-module is finitely generated in fact implies it is cyclic. This follows since by taking a common denominator and removing numerators, if $\frac{1}{f^{k}}$ is the smallest negative power of $f$ in a generating set, it must then generate as higher powers of $f$ can be obtained by multiplication by elements of $R$. In characteristic 0 , the exact power of $f$ which generates this localization as a $D$-module depends on the roots of a certain polynomial called the Berstein-Sato polynomial. In characteristic $p$ positive, and for

\footnotetext{
${ }^{5} \mathrm{~A} k$-differential operator of order $n$ of a $k$-algebra $R$ is defined inductively. A $k$-differential operator of order 0 is multiplication by an element of $R$. A $k$-differential operator of order $n$ is a $k$-linear map $d: R \rightarrow R$ such that for every $r \in R$, the commutator $[d, r]$ is a $k$-differential operator of order $n-1$.
} 
the polynomial ring case, Alvares-Montaner, Blickle, and Lyubeznik [1] have recently proved the surprising result that $\frac{1}{f}$ generates $R_{f}$ as a $D$-module.

\subsection{Exercises.}

EXERCISE 21. Let $(R, \mathfrak{m})$ be a Noetherian local ring and $I$ an ideal. If $H_{I}^{q}(R)=0$ for all $q>n$, prove that $H_{I}^{q}(M)=0$ for all $R$-modules $M$, and for all $q>n$.

EXERCISE 22. Suppose that $A$ is an $n$ by $n+1$ matrix whose first $n$ columns give an $n$ by $n$ symmetric matrix $C$. Concatenate $A$ and its transpose, and filling out this $n+1$ by $n+1$ matrix with a 0 to make a matrix $B$. Prove that $\operatorname{det}(B)$ and $\operatorname{det}(C)$ generate up to radical the ideal generated by all of the maximal minors of $A$.

EXERCISE 23. Let $k$ be a field, and $R=k\left[x_{1}, \ldots, x_{n}\right] /(F)$ a hypersurface, where $F$ is a homogeneous polynomial of degree $d$. Prove that the a-invariant of $R$ is $d-n$.

EXERCISE 24. $R$ be a Noetherian ring and $I$ an ideal of $R$. Set $n=c d(R, I)$. For every $R$-module $M$, prove that $H_{I}^{n}(M) \cong H_{I}^{n}(R) \otimes_{R} M$.

EXERCISE 25. Let $R$ be a local Noetherian ring of dimension d at least 2, and let $I \subseteq J$ be ideals. Prove that $H_{J}^{d}(R)$ maps onto $H_{I}^{d}(R)$. (Hint: use the sequence of Theorem 2.2.)

Exercise 26. Let $R$ be a local Cohen-Macaulay ring. Prove directly that the highest local cohomology module $H_{\mathfrak{m}}^{d}(R) \neq 0$ ( $d$ is the dimension of $R$ ) as follows: choose a system of parameters $x_{1}, \ldots, x_{d}$ and use the fact they form a regular sequence, together with the identification of $H_{\mathfrak{m}}^{d}(R)$ with the top Koszul cohomology of $R$ with respect to $x_{1}, \ldots, x_{d}$, to prove that the image of $\frac{1}{x_{1} \cdots x_{d}}$ in this top cohomology must be nonzero.

Exercise 27. Use Exercise 22 to prove that the ideal $P$ in $k\left[x_{1}, x_{2}, x_{3}, x_{4}\right]$ defining the 'twisted cubic' $k\left[t^{3}, t^{2} s, t s^{2}, s^{3}\right]$ is generated up to radical by 2 elements.

EXERCISE 28. Let $R$ be a local Noetherian ring of dimension $d$, and let $x_{1}, \ldots, x_{s}$ be elements of $R$ generating an ideal $I$. Prove that $H_{I}^{s}(R)=0$ if and only if the following condition is satisfied: for all $p>0$, there exists $q$ such that

$$
\left(x_{1} \cdots x_{s}\right)^{q} \in\left(x_{1}^{p+q}, \ldots, x_{s}^{p+q}\right) .
$$

(Hint: Think about what it means for the image of the element $\frac{1}{\left(x_{1} \cdots x_{s}\right)^{p}}$ in the highest Koszul cohomology to be zero.)

EXERCISE 29. Prove that for arbitrary polynomials $f, g, h \in k[x, y]=R$ ( $k$ a field), $(f g h)^{2} \in$ $\left(f^{3}, g^{3}, h^{3}\right)$. Assuming this, prove that $H_{(f, g, h)}^{3}(R)=0$, using Exercise 28.

Exercise 30. Prove the statement of Proposition 5.10.

\section{Vanishing Theorems II}

In this section we prove two basic and important theorems. The first gives necessary and sufficient conditions for the local cohomology $H_{I}^{d}(R)$ to vanish, where $d$ is the dimension of the local ring $R$. We then apply this theorem together with the knowledge of the structure of $H_{\mathfrak{m}}^{d}(R)$ to prove a strong connectedness theorem due to Grothendieck. The first result is usually called the "Hartshorne-Lichtenbaum Vanishing Theorem" (HLVT for short). See [16]. A good discussion also appears in [17], Chapter III. The proof we give is from $[\mathbf{6}]$. 
THEOREM 6.1. (HLVT) Let $(R, \mathfrak{m})$ be a local Noetherian ring and let $I$ be an ideal of $R$. Set $d=\operatorname{dim}(R)$. Then the following statements are equivalent:

(1) $H_{I}^{d}(R)=0$.

(2) For all minimal primes $P$ of $\widehat{R}$ with $\operatorname{dim}(\widehat{R} / P)=\operatorname{dim}(R), \operatorname{dim}(\widehat{R} /(I \widehat{R}+P))>0$.

Proof. The easy direction is $(1) \Longrightarrow(2)$. For suppose that $(2)$ does not hold. Without loss of generality we may assume that $R$ is complete and that there is a minimal prime $P$ of maximal dimension in $R$ such that $I+P$ is primary to the maximal ideal. In particular, $H_{(I+P) / P}^{d}(R / P)=$ $H_{\mathfrak{m} / P}^{d}(R / P) \neq 0$ by Theorem 5.9. By the Independence of Base, this local cohomology is the same as $H_{I}^{d}(R / P)$. But $R$ maps onto $R / P$, and then the long exact sequence on local cohomology proves that $H_{I}^{d}(R)$ maps onto $H_{I}^{d}(R / P)$, since the cokernel embeds in a $(d+1)^{s t}$ local cohomology module, which we know is zero. Thus $H_{I}^{d}(R) \neq 0$, a contradiction.

The harder direction is $(2) \Longrightarrow(1)$.

For simplicity, we give the proof in the case in which the ring contains a field, though the general case is not much harder. We first reduce to the case in which $R$ is a complete domain, and $I=P$ is a prime of height $d-1$.

Suppose by way of contradiction that under the conditions of $(2), H_{I}^{d}(R) \neq 0$. Since $H_{I \widehat{R}}^{d}(\widehat{R})=$ $H_{I}^{d}(R) \otimes_{R} \widehat{R}$, it follows that $H_{I \widehat{R}}^{d}(\widehat{R}) \neq 0$. Therefore we may first complete $R$. As $H_{I}^{d}(R) \neq 0$, it is easy to see this is true modulo some minimal prime of $R$ of maximal dimension (Exercise 34). Hence we may assume $R$ is a complete local domain. Choose $I$ maximal such that $H_{I}^{d}(R) \neq 0$ but $\operatorname{dim}(R / I)>0$; if $I$ is not a prime of dimension 1 we may choose $x \notin I$ such that $\operatorname{dim}(R /(I, x))>0$; in this case the exact sequence

$$
\cdots \longrightarrow H_{(I, x)}^{i}(R) \longrightarrow H_{I}^{i}(R) \longrightarrow H_{I_{x}}^{i}\left(R_{x}\right) \longrightarrow \cdots
$$

at $i=d$ shows that $H_{(I, x)}^{d}(R) \neq 0\left(\right.$ as $\left.\operatorname{dim} R_{x}<d\right)$, contradicting the maximality of $I$.

We next reduce to the case in which $R$ is a Gorenstein ring. Let $k$ be a coefficient field of $R$, i.e. a subfield of $R$ which maps isomorphically onto the residue field.

Choose $x_{1}, \ldots, x_{d} \in P$ such that $P=\sqrt{\left(x_{1}, \ldots, x_{d}\right)}$. Such $x_{1}, \ldots, x_{d}$ can be chosen as follows: first choose $x_{1}, \ldots, x_{d-1}$ in $P$ such that $h t\left(x_{1}, \ldots, x_{d-1}\right)=d-1$. Choose $x_{d}$ in $P$ which is not in the union of the other minimal primes containing $x_{1}, \ldots, x_{d-1}$. Clearly $\sqrt{\left(x_{1}, \ldots, x_{d}\right)}=P$ in this case, since the only primes containing $x_{1}, \ldots, x_{d-1}$ are the maximal ideal and the primes minimal over the ideal they generate. Choose $y \notin P$ such that $x_{1}, \ldots, x_{d-1}, y$ is a full system of parameters. Set $A=k\left[\left[x_{1}, \ldots x_{d-1}, y\right]\right] . A$ is a complete regular local ring, and $R$ is a finite $A$-module. Finally let $B=A\left[x_{d}\right]$. As $A \subseteq B \subseteq R, B$ is necessarily finite over $A$, hence both complete and local. Furthermore $B$ is Gorenstein since it is a hypersurface ring. This can seen as follows: Map a polynomial ring $A[T]$ onto $B$ by sending $T$ to $x_{d}$. The kernel is a prime ideal $p$ of $A[T]$, and the height of $p$ must be exactly one, since the dimension of $B$ is the same as the dimension of $A$. But $A$ is a UFD, and therefore so is $A[T]$. Every height one prime in a UFD is principal. Therefore $p=(f)$ is principal, and $B=A[T] /(f)$ is a hypersurface, hence Gorenstein. Set $Q=P \cap B$. Note that $H_{P}^{d}(R)=H_{\left(x_{1}, \ldots, x_{d}\right) R}^{d}(R)$ as $P=\sqrt{\left(x_{1}, \ldots, x_{d}\right)}$. We claim that $Q=\sqrt{\left(x_{1}, \ldots x_{d}\right) B}$. Certainly $Q$ contains $\left(x_{1}, \ldots x_{d}\right) B$ so it suffices to prove that every prime in $B$ containing $\left(x_{1}, \ldots x_{d}\right) B$ contains $Q$. Let $Q^{\prime}$ be such a prime. As the extension $\operatorname{ring} R$ is module-finite over $B$, there is a prime $P^{\prime}$ in $R$ which contracts to $Q^{\prime}$ by the Lying Over theorem. Since $P^{\prime}$ contains $\left(x_{1}, \ldots x_{d}\right) R$, it must be either $P$ or $\mathfrak{m}$. If it is $P$, it contracts to $Q$, and $Q^{\prime}=Q$. Otherwise, $Q^{\prime}$ is the maximal ideal of $B$ and contains $Q$.

$$
\begin{gathered}
\text { Hence } H_{Q}^{d}(B)=H_{\left(x_{1}, \ldots, x_{d}\right) B}^{d}(B) . \text { If } 0=H_{Q}^{d}(B), \text { then } 0=H_{\left(x_{1}, \ldots, x_{d}\right) B}^{d}(B) \text { and } \\
0=H_{\left(x_{1}, \ldots, x_{d}\right) B}^{d}(B) \otimes_{B} R \cong H_{\left(x_{1}, \ldots, x_{d}\right) R}^{d}(R)=H_{P}^{d}(R) .
\end{gathered}
$$


(The middle isomorphism follows as tensor is right exact and $H_{J}^{j}(M)=0$ for $j>d$ for any ideal $J$ of $B$ and any $B$-module $M$.) It suffices to prove that $0=H_{Q}^{d}(B)$; we relabel to assume that $R$ is Gorenstein and $P$ is a prime ideal of height $d-1$. We need to prove that $H_{P}^{d}(R)=0$.

Write $P^{n}=P^{(n)} \cap J_{n}$, where $J_{n}$ is $\mathfrak{m}$-primary, and $P^{(n)}=P^{n} R_{P} \cap R$. As $R$ is a domain, $\cap_{n \geq 0} P^{(n)}=(0)$ and Chevalley's Theorem ${ }^{6}$ shows that each $J_{n}$ contains some $P^{(k)}$, which shows that $\left\{P^{n}\right\}$ and $\left\{P^{(n)}\right\}$ are cofinal.

Hence $H_{P}^{d}(R)=\lim _{\longrightarrow} \operatorname{Ext}_{R}^{d}\left(R / P^{(n)}, R\right)=0$ since these modules are the Matlis duals of $H_{\mathfrak{m}}^{0}\left(R / P^{(n)}\right)$, and by definition of symbolic powers, these modules are all 0 .

EXAMPLE 6.2. To illustrate this theorem, let $k$ be a field of characteristic 0 , and let $R=$ $(k[x, y, u, v] /(f))_{\mathfrak{m}}$, where $f=x y-u x^{2}-v y^{2}$ and $\mathfrak{m}=(x, y, u, v)$. Set $P=(y, u, v) R$. It is easy to prove that $R$ is a three-dimensional domain, and $P$ is a height two prime ideal of $R$. Is $H_{P}^{3}(R)=0$ ? After completion one can prove that $f$ factors (Exercise 32) into two power series $f=(x-v y+\ldots)(y-u x+\ldots)$, where every term in the element $x-v y+\ldots$ lies in $(y, u, v)$ except for the first term $x$. This means there are two minimal primes lying over 0 in $\widehat{R}$, and $P \widehat{R}+(x-v y+\ldots)=\mathfrak{m}$. HLVT then implies that $H_{P}^{3}(R) \neq 0$.

There are various generalizations of this vanishing theorem. We state one to give a sample:

TheOREM 6.3. (see [32] Cor. 2.11, [33] III, 5.5) Let $R=k\left[\left[x_{1}, \ldots, x_{n}\right]\right]$ be a formal power series ring over a separably closed field $k$. Let $I$ be a proper ideal of $R$. Then the following are equivalent:

(1) $\operatorname{cd}(R, I)<n-1$.

(2) $\operatorname{dim}(R / I)>1$, and $\operatorname{Spec}(R) \backslash\{\mathfrak{m}\}$ is connected.

Theorem 6.1 has a beautiful application to connectedness properites of Spec, given below. Let $(R, \mathfrak{m})$ be a local ring, and let $X$ be the punctured spectrum, i.e., $\operatorname{Spec}(R) \backslash\{\mathfrak{m}\}$. We first interpret ideal-theoretically what it means for this open set to be disconnected. If this set is disconnnected, there exists nonempty clopen sets $U \subseteq X$ and $W \subseteq X$ such that $U \cap W=\emptyset$ and $U \cup W=X$. By definition, $U=V(I) \cap X$ and $W=V(J) \cap X$ for some ideals $I$ and $J$ in $R$. The set $U \cap W$ is given by $X \cap V(I+J)$, while the set $U \cup W$ is given by $V(I \cap J) \cap X$. The conditions mean that $I+J$ is $\mathfrak{m}$-primary, and $I \cap J$ is in the nilradical of $R$, i.e., is in every prime ideal of $R$. The condition that $U$ and $W$ are non-empty means that neither $I$ nor $J$ is $\mathfrak{m}$-primary.

THEOREM 6.4. (Connectedness Theorem) Let $(R, \mathfrak{m}, k)$ be an analytically irreducible local ring of dimension $n$, and let $\mathfrak{A}$ be an ideal of $R$ generated by at most $n-2$ elements. Then the punctured spectrum of Spec $R / \mathfrak{A}$ is connected.

REMARK 6.5. We do not even need the condition that $\mathfrak{A}$ be generated by $n-2$ or fewer elements: all that is needed is that $H_{\mathfrak{A}}^{n-1}(R)=H_{\mathfrak{A}}^{n}(R)=0$, and the second condition is automatic if $R$ is a complete local domain and $\mathfrak{A}$ is not $\mathfrak{m}$-primary.

In $[7]$ a clever proof was given, whose outline we shall follow here.

Proof. If not let $I, J$ be ideals which give a disconnection, so that $I \cap J$ has the same radical as $\mathfrak{A}, I+J$ is primary to $\mathfrak{m}$, but neither $I$ nor $J$ is primary to $\mathfrak{m}$. The Mayer-Vietoris sequence for local cohomology then yields:

$$
\cdots \longrightarrow H_{I \cap J}^{n-1}(R) \longrightarrow H_{I+J}^{n}(R) \longrightarrow H_{I}^{n}(R) \oplus H_{J}^{n}(R) \longrightarrow H_{I \cap J}^{n}(R) \longrightarrow \cdots
$$

\footnotetext{
${ }^{6}$ Chevalley's result says that if $(R, \mathfrak{m})$ is a complete local domain, and $\left\{I_{n}\right\}$ is a decreasing chain of ideals such that $\cap_{n} I_{n}=0$, then $I_{n} \subseteq \mathfrak{m}^{k(n)}$, where $k(n)$ goes to infinity.
} 
and the first and last terms displayed are zero, since $I \cap J$ has the same radical as an ideal with at most $n-2$ generators. Since $I+J$ is primary to $\mathfrak{m}$, the sequence yields an isomorphism:

$$
H_{\mathfrak{m}}^{n}(R) \cong H_{I}^{n}(R) \oplus H_{J}^{n}(R) .
$$

But the Hartshorne-Lichtenbaum vanishing theorem implies that both $H_{I}^{n}(R)$ and $H_{J}^{n}(R)$ are zero, since $R$ is a complete domain and neither $I$ nor $J$ is $\mathfrak{m}$-primary. Since $H_{\mathfrak{m}}^{n}(R) \neq 0$ by Theorem 5.9, this contradiction finishes the proof.

6.1. Applications to Intersections of Algebraic Varieties. In this section we'll give an application of local cohomology to the topology of certain algebraic sets. Our main result is one of Fulton and Hansen [13]:

TheOREM 6.6. Let $k$ be an algebraically closed field and suppose that $X \subseteq \mathbb{P}_{k}^{n}$ and $Y \subseteq \mathbb{P}_{k}^{n}$ are algebraic varieties. If $\operatorname{dim}(X)+\operatorname{dim}(Y)>n$, then $X \cap Y$ is connected.

Another result, due to Kalkbrenner and Sturmfels, is again about the topology of certain algebraic sets. Let $P \subseteq k\left[x_{0}, \ldots, x_{n}\right]$ be a homogeneous prime ideal. Fix a term ordering on the monomials, and let $\operatorname{in}(P)=I$ be the initial ideal of $P$ with respect to this ordering. The theorem of Kalkbrenner and Sturmfels [22, Theorem 1] states:

Theorem 6.7. Let $P \subseteq R=k\left[x_{1}, x_{2}, \ldots, x_{r}\right]$ be a prime ideal and $<$ any monomial order. Then $R / \sqrt{i n_{<}(P)}$ is equidimensional and connected in codimension one.

Appendix 1, written by Amelia Taylor, provides a slightly different proof of the this theorem from that of Kalkbrenner and Sturmfels, one which relies on our main connectedness theorem.

The dimension restriction in the theorem of Fulton and Hansen is clearly necessary: if $\operatorname{dim}(X)+$ $\operatorname{dim}(Y)<n$, then in general we would not expect $X$ and $Y$ to meet at all as there is plenty of room for both of them. If $\operatorname{dim}(X)+\operatorname{dim}(Y)=n$, in general $X \cap Y$ would meet in a set of dimension 0 , i.e., in a set of points, and this set is disconnected if there is more than one point.

Proof. We first change the geometric language in the theorem of Fulton and Hansen to algebraic statements. The scheme $\mathbb{P}_{k}^{n}$ consists as a set of all homogeneous primes of the polynomial ring $k\left[x_{0}, \ldots, x_{n}\right]$ except the origin $\mathfrak{m}=\left(x_{0}, \ldots, x_{n}\right)$. The closed sets are defined as in the Zariski topology, but where we only use homogeneous ideals. Because of this, we can reinterpret the theorem as a purely local statement. Let $X$ be the set of homogeneous primes containing a fixed homogeneous prime $P$, and $Y$ be the set of homogeneous primes containing a fixed homogeneous prime $Q$. The homogeneous coordinate ring of $X$ is the ring $k\left[x_{0}, \ldots, x_{n}\right] / P$, and that of $Y$ is $k\left[x_{0}, \ldots, x_{n}\right] / Q$. The dimension of $X$ is one less that the Krull dimension of $k\left[x_{0}, \ldots, x_{n}\right] / P$ since we have removed the maximal ideal $\left(x_{0}, \ldots, x_{n}\right)$. Similarly, the dimension of $Y$ is one less than the dimension of $k\left[x_{0}, \ldots, x_{n}\right] / Q$. An ideal defining the set $X \cap Y$ is the ideal $P+Q$. To say it is disconnected means there are homogeneous ideals $I$ and $J$ such that $I+J$ is primary to $\mathfrak{m}, P+Q \subseteq I \cap J$, and $\sqrt{P+Q}=\sqrt{I \cap J}$, but neither $I$ nor $J$ is $\mathfrak{m}$-primary.

We use "reduction to the diagonal" to prove this theorem. Namely, consider the homogeneous coordinate ring of the "join" of $X$ and $Y$, namely, the product of their respective homogeneous coordinate rings: $R=k\left[x_{0}, \ldots, x_{n}\right] / P \otimes_{k} k\left[x_{0}, \ldots, x_{n}\right] / Q . R$ is a domain since $k$ is algebraically closed (Exercise 38). We set $l_{i}=X_{i}-Y_{i}$, so that the ideal generated by these linear forms defines the diagonal. The dimension of $R$ is $\operatorname{dim}(X)+1+\operatorname{dim}(Y)+1>n+2$. Notice that $R /\left(l_{0}, \ldots, l_{n}\right) \cong$ $k\left[x_{0}, \ldots, x_{n}\right] /(P+Q)$. Suppose that $X \cap Y$ is disconnected, and let $I$ and $J$ be as above, but moved into $R$ via the isomorphism $R /\left(l_{0}, \ldots, l_{n}\right) \cong k\left[x_{0}, \ldots, x_{n}\right] /(P+Q)$. As ideals of $R, I$ and $J$ satisfy the following: $I+J$ is primary to $\mathfrak{m}_{R},\left(l_{0}, \ldots, l_{n}\right) \subseteq I \cap J$ and has the same nilradical, and neither $I$ nor $J$ is primary to the maximal homogeneous ideal $\mathfrak{m}_{R}$. These properties remain true after completing 
$R$ at $\mathfrak{m}_{R}$; we denote this ring by $S$. We use the grading to prove that $S$ is a domain. This is true because the associated graded ring of $S$ is the same as the associated graded ring of $R$ with respect to $\mathfrak{m}_{R}$, and this ring is isomorphic to $R$ since $R$ is graded and generated by 1 -forms. (See Exercise 35 and Appendix 1).

Thus $S$ is a complete local domain of dimension equal to the dimension of $R$. Moreover, by construction the punctured spectrum of $S /\left(l_{0}, \ldots, l_{n}\right) S$ is disconnected. Since $(n+1)+2=n+3 \leq$ $\operatorname{dim}(S)$, we can apply the connectedness theorem to reach a contradiction.

The next two subsections give some further results on the vanishing of local cohomology in the multigraded situation and in positive characteristic. We do not give proofs in these sections.

6.2. The multi-graded case. In recent years, there has been great interest in the local cohomolgy of polynomial rings with support in an ideal generated by monomials. This interest arose through considerations coming from the study of toric varieties. In this case we can consider the ring as multigraded, and study the local cohomology through a Koszul-like complex called the Taylor resolution. A duality then immerges. We state one result (see for example [35]):

THEOREM 6.8. Let $I$ be an ideal generated by square-free monomials in a polynomial ring $R$ over a field. Let $\mathfrak{m}$ be the irrelevant ideal. Then $H_{I}^{j}(R)=0$ if and only if $H_{\mathfrak{m}}^{n-j}(R / I)=0$.

Notice that the local cohomology $H_{I}^{j}(R)$ only depends on the radical of $I$, so there is no loss of generality in replacing an arbitrary monomial ideal by its radical, which is always generated by square-free monomials. However the right-hand side cohomology in the theorem above will in general depend on the radical of $I$.

6.3. Positive Characteristic. Suppose now that $(R, \mathfrak{m})$ is a regular local ring of positive characteristic $p$. The Frobenius map $F: R \rightarrow R$ is the homomorphism that sends an element to its $p$ th power. It is a fundamental and enormously powerful fact that this map truly is an endomorphism. The Frobenius likewise acts on localizations of $R$, and hence acts on the cohomology of the Koszul type complexes which give an alternate definition of local cohomology. In particular, the Frobenius acts on local cohomology. In many ways the Frobenius action and the situation for monomial ideals parallel each other, partly because one can raise relations (or more generally syzygies) to the $p$ th power component wise and still obtain significant information. A result of Lyubeznik is quite close to the multigraded theorem above:

THEOREM 6.9. Let $(R, \mathfrak{m})$ be a regular local ring of characteristic $p$ and dimension $n$, let $I$ be an ideal in $R$, and set $A=R / I$. Then $H_{I}^{j}(R)=0$ if and only if there exists an integer such that the map given by s-iterates of Frobenius $F^{s}: H_{\mathfrak{m}}^{n-j}(A) \rightarrow H_{\mathfrak{m}}^{n-j}(A)$ is the zero map.

The significance of this theorem and the one stated in the multigraded section above is that it changes a difficult question on the vanishing of local cohomology with support in an arbitrary ideal $I$ to a much more tractable question concerning the local cohomology of a quotient ring with respect to the maximal ideal. One then has local duality which can in turn change this question into one about Ext modules which are finitely generated and can be studied from the free resolution of the quotient ring over the regular base ring.

These results are not altogether unexpected. As mentioned above, the Matlis dual of $H_{\mathfrak{m}}^{n-j}(A)$ is $\operatorname{Ext}_{R}^{j}(R / I, R)$, and this module maps to the local cohomology module $H_{I}^{j}(R)=\lim _{\longrightarrow} \operatorname{Ext}_{R}^{j}\left(R / I^{n}, R\right)$. In both the theorem of Lyubeznik and the multigraded case, there is a Frobenius-like action on the free resolution of $R / I$ which give ideals which are cofinal with the powers of $I$ and can be used to compute $H_{I}^{j}(R)$. While this is not a proof, it does provide evidence of why one might expect such results. 
Even more can be said.

THEOREM 6.10. Let $(R, \mathfrak{m})$ be a regular local ring of characteristic $p$ and dimension $n$, let $I$ be an ideal in $R$. Set $t=\operatorname{depth}(A)$. Then $H_{I}^{j}(R)=0$ for all $j>n-t$. In particular, if $A$ is Cohen-Macaulay then $H_{I}^{j}(R)=0$ for all $j \neq c$, where $c$ is the height of $I$.

The proof of the first part of this theorem rests on the flatness of the Frobenius map over regular local rings of positive characteristic. The second part follows from the first part and the fact that $H_{I}^{j}(R)=0$ for all $j<c$ as the $I$-depth of $R$ is $c$.

\subsection{Exercises.}

EXERCISE 31. Prove the result of Chevalley: if $(R, \mathfrak{m})$ is a complete local domain, and $\left\{I_{n}\right\}$ is a decreasing chain of ideals such that $\cap_{n} I_{n}=0$, then $I_{n} \subseteq \mathfrak{m}^{k(n)}$, where $k(n)$ goes to infinity.

ExERCISE 32. Prove the claim in Example 4.2 concerning the factorization of $x y-u x^{2}-v y^{2}$ into power series.

EXERCISE 33. Let $R=k[[x, y, u, v]] /(x u-y v)$, and $I=(x, y) R$. Prove that $H_{I}^{3}(R)=0$, but $H_{I}^{2}(R) \neq 0$.

EXERCISE 34. Let $R$ be a local Noetherian ring of dimension $d$, and let $I$ be an ideal of $R$. If $H_{I}^{d}(R) \neq 0$, prove this is true modulo some minimal prime of $R$ of maximal dimension.

EXERCISE 35. Prove the claim in the text that if $R$ is a standard graded algebra with irrelevant ideal $\mathfrak{m}$, then the associated graded ring of $R$ is isomorphic to $R$.

EXERCISE 36. Let $(R, \mathfrak{m})$ be a Noetherian local ring. If the associated graded ring of $R$ is a domain, prove that $R$ is a domain.

Exercise 37. Let $R$ be a Noetherian domain, $I$ an ideal and $U=\operatorname{Spec}(R) \backslash V(I)$. Prove there is a short exact sequence

where $S=\cap_{p \in U} R_{p}$.

$$
0 \rightarrow R \rightarrow S \rightarrow H_{I}^{1}(R) \rightarrow 0
$$

EXERCISE 38. Let $k$ be an algebraically closed field and let $R$ and $S$ be two finitely generated $k$-algebras which are domains. Prove that $R \otimes_{k} S$ is also a domain. 


\section{Appendix 1: Using local cohomology to prove a result of Kalkbrenner and Sturmfels}

\section{by Amelia Taylor}

7.1. Introduction. Let $I$ be an ideal in $R=k\left[x_{1}, x_{2}, \ldots, x_{r}\right]$. A monomial order $<$ on $R$ is a total order on the monomials in $R$ such that $1 \leq m$ for each monomial $m$ in $R$ and if $a, b, c$ are monomials in $R$ with $a<b$ then $c a<c b$. A monomial order on a polynomial ring in several variables generalizes the notion of degree for a polynomial ring in one variable. The initial term of an element $f \in R$, denoted $i n_{<}(f)$, is the largest term of $f$ with respect to a fixed monomial order. Given an ideal $I$ of $R$, the initial ideal is $i n_{<}(I)=(\{i n(f): f \in I\})$. Different monomial orders may yield different initial ideals, so whenever an initial ideal is referred to, it is assumed a monomial order has been fixed.

In 1988 Kredel and Weispfenning [23, p. 234] conjectured that if $P$ is a prime ideal in $R$, then $R / \sqrt{i n_{<}(P)}$ is equidimensional. In 1995 Kalkbrenner and Sturmfels proved the following stronger theorem.

Theorem 7.1. [22, Theorem 1] Let $P \subseteq R=k\left[x_{1}, x_{2}, \ldots, x_{r}\right]$ be a prime ideal and $<$ any monomial order. Then $R / \sqrt{i n_{<}(P)}$ is equidimensional and connected in codimension one.

For a square-free monomial ideal $I \subseteq R, R / I$ is reduced and the minimal prime ideals of $R / I$ are generated by subsets of the variables. In this context $R / I$ is connected in codimension one, if given any two prime ideals, $P_{1}, P_{2}$, minimal in $k\left[x_{1}, x_{2}, \ldots, x_{r}\right] / I$, there exists a chain of minimal prime ideals, $P_{1}=Q_{1}, Q_{2}, \ldots, Q_{s}=P_{2}$, such that $\operatorname{codim}\left(Q_{i}, Q_{i+1}\right)=\operatorname{codim}\left(Q_{i}\right)+1$, for $1 \leq i \leq s-1$.

Kalkbrenner and Sturmfels, and Kredel and Weispfenning, use the language of simplicial complexes in their papers and we introduce that language here. A finite abstract simplicial complex is a set, $\Delta$, of subsets of $X=\left\{x_{1}, \ldots, x_{r}\right\}$ such that $x_{i} \in \Delta$ for $1 \leq i \leq r$ and if $U \in \Delta$ and $V \subseteq U$ then $V \in \Delta$. The name is intended to emphasize that the maps usually associated with simplicial complexes do not play a role here. One can assume the trivial maps if needed, and since confusion rarely arises on this point in commutative algebra, they are usually called just simplicial complexes. Let $I$ be an ideal in $k\left[x_{1}, x_{2}, \ldots, x_{r}\right]$. A set $U \subseteq\left\{x_{1}, \ldots, x_{r}\right\}=X$ is independent $\bmod I$ if $I \cap k[U]=(0)$. The sets are said to be independent because this notion for ideals is a generalization of linear independence in a vector space. The collection of all the subsets of $X$ that are independent $\bmod I, \Delta(I)=\{U: I \cap k[U]=(0)\}$, forms a simplicial complex called the independence complex of $I$. Given a simplicial complex $\Delta$ on $X=\left\{x_{1}, \ldots, x_{r}\right\}$, the ideal defined by $I_{\Delta}=\left\{x_{i_{1}} \cdots x_{i_{t}} \mid\left\{x_{i_{1}}, \ldots, x_{i_{t}}\right\} \notin \Delta\right\}$ is a square-free monomial ideal. The ring $k[X] / I_{\Delta}$ is the face ring or Stanley-Reisner ring for $\Delta[\mathbf{3 7}]$.

These two constructions give a correspondence between simplicial complexes and square-free monomial ideals. In particular, for any simplicial complex, $\Delta\left(I_{\Delta}\right)=\Delta$ and for any square-free monomial ideal, $I_{\Delta(I)}=I$.

The sets in $\Delta$ are called faces. A face is maximal if it is not properly contained in another face. Maximal faces are often called facets. Let $F=\left\{x_{i_{1}}, \ldots, x_{i_{t}}\right\}$ be a facet of a simplicial complex $\Delta$. Then $\left(X \backslash\left\{x_{i_{1}}, \ldots, x_{i_{t}}\right\}\right)$ is a minimal prime ideal for $I_{\Delta}$. Suppose $I_{\Delta} \nsubseteq\left(X \backslash\left\{x_{i_{1}}, \ldots, x_{i_{t}}\right\}\right)$, then there exists a monomial $m$ in $I$ such that $x_{i_{j}}$ divides $m$ for some $1 \leq j \leq t$. In this case, $m \in I_{\Delta} \cap k[F]$, contradicting the fact that $F$ is in $\Delta$. Now suppose that $\left(X \backslash\left\{x_{i_{1}}, \ldots, x_{i_{t}}\right\}\right)$ is not minimal. Then for some $i_{j}, 1 \leq j \leq t, x_{i_{j}}$ does not divide any generator of $I_{\Delta}$. Hence $k\left[F \cup\left\{x_{i_{j}}\right\}\right] \cap I_{\Delta}=(0)$ and $F$ is not a facet in $\Delta$; a contradiction. Thus facets in $\Delta$ correspond to prime ideals minimal over $I_{\Delta}$. Similarly, prime ideals minimal over $I$ correspond to facets of $\Delta(I)$.

A simplicial complex is called pure if all of the facets have the same cardinality and strongly connected if given any two facets $F, G \in \Delta$, there exists a chain of facets $F=F_{0}, F_{1}, \ldots, F_{s}=G$ 
in $\Delta$ such that $\left|F_{i-1} \backslash F_{i}\right|=1$ for $1 \leq i \leq s$. The correspondence given above for facets and minimal prime ideals implies that if $I$ is a square-free monomial ideal, then $k\left[x_{1}, x_{2}, \ldots, x_{r}\right] / I$ is equidimensional and connected in codimension one if and only if $\Delta(I)$ is pure and strongly connected. Equivalently, a simplicial complex $\Delta$ is pure and strongly connected if and only if its StanleyReisner ring $k\left[x_{1}, x_{2}, \ldots, x_{r}\right] / I_{\Delta}$ is equidimensional and connected in codimension one. The original statement in Kalkbrenner and Sturmfels' paper is that the independence complex of the initial ideal of a prime ideal is pure and strongly connected.

The result of Kalkbrenner and Sturmfels concerns the initial ideal of a prime ideal, and since initial ideals depend on the order chosen, the order chosen can affect the geometry, or topology of the initial ideal. Since their result is stated without regard to the monomial order, any proof of this result must be independent of the monomial order. Kalkbrenner and Sturmfels use weight vectors to distinguish between different monomial orders, and ultimately, to prove the result independent of the monomial order. In their proof they first prove the result for the weight vector $(1,1, \ldots, 1)$ which, depending on the ideal, corresponds to a particular monomial order, and allows them to assume that the given prime ideal is homogeneous. One key step in this first case, is the Fulton-Hanson connectedness theorem [13], Corollary 1 . They then use this case to complete the proof for arbitrary weight vectors, and hence arbitrary monomial orders. In the proof given here we only need to reduce to the case where we assume that the prime ideal is quasi-homogeneous, thus we obtain the proof for arbitrary weight vectors directly. Once in the quasi-homogeneous case we use the connectedness theorem, Theorem 4.4.

In Section 7.2, we collect some standard results about quasi-homogeneous polynomials and ideals, which we include for the reader's convenience. Most of these results can be found in section 10.3 of Becker and Weispfenning's book [3], but the language and notation they use is consistent with that in their book and we feel that our language better fits the language in the remainder of this paper. We also include, in this section, Lemmas 2 and 3 from Kalkbrenner and Sturmfels' paper [22]. For Lemma 2 they reference a paper of Mora and Robbiano [31]; we include a proof. We include a proof of lemma 3 that is modified, but fundamentally the same as the proof given in [22]. In Section 7.3 we give the proof of Kalbrenner and Sturmfels' result as an application of the connectedness Theorem 6.4.

7.2. Quasi-Homogenization. We begin with some basic results on weight vectors. Let $f \in$ $k\left[x_{1}, \ldots, x_{r}\right]$, and $\omega \in \mathbb{N}^{r}$. Define $i n_{\omega}(f)$ to be the leading coefficient of $f\left(t^{\omega_{1}} x_{1}, \ldots, t^{\omega_{r}} x_{r}\right)$ as a polynomial in $t$. Note that $i n_{\omega}(f)$ is an element of $k\left[x_{1}, \ldots, x_{r}\right]$ and is not necessarily monomial. Let $i n_{\omega}(I)=\left\{i n_{\omega}(f): f \in I\right\}$. The vector $\omega$ represents $<$ for the ideal $I$ if $i n_{<}(I)=i n_{\omega}(I)[\mathbf{2 2}]$. For any monomial order $<$ and any ideal $I \subset R=k\left[x_{1}, \ldots, x_{r}\right]$, there exists a non-negative integer vector $\omega \in \mathbb{N}^{n}$ such that $\omega$ represents $<[38$, Proposition 1.11].

Lemma 7.2. [22] Let $G=\left\{g_{1}, \ldots, g_{m}\right\}$ be the reduced Gröbner basis for I with respect to a monomial order $<$. A vector $\omega \in \mathbb{N}^{r}$ represents $<$ for $I$, if and only if in ${ }_{<}\left(g_{i}\right)=i n_{\omega}\left(g_{i}\right)$ for $1 \leq i \leq m$.

Proof. Assume $\omega$ represents $<$ for the ideal $I$. Then

$$
i n_{\omega}(I)=i n_{<}(I)=\left(i n_{<}\left(g_{1}\right), \ldots, i n_{<}\left(g_{m}\right)\right) .
$$

Since $g_{1}, \ldots g_{m}$ form a reduced Gröbner basis, no term of $g_{i}$ is divisible by $i n_{<}\left(g_{j}\right)$ for all $i \neq j$. For fixed $i, 1 \leq i \leq m, i n_{\omega}\left(g_{i}\right) \in i n_{\omega}(I)=i n_{<}(I)$. Hence there exist $f_{1}, \ldots, f_{m} \in R$ such that

$$
i n_{\omega}\left(g_{i}\right)=f_{1} i n_{<}\left(g_{1}\right)+\cdots+f_{m} i n_{<}\left(g_{m}\right) .
$$

While $i n_{\omega}\left(g_{i}\right)$ may not be $i n_{<}\left(g_{i}\right)$, it is some terms of $g_{i}$. The above equation implies that each term of $i n_{\omega}\left(g_{i}\right)$ is divisible by some $i n_{<}\left(g_{j}\right)$. Since $i n_{\omega}\left(g_{i}\right)$ consists of terms of $g_{i}$ and $G$ is a reduced 
Gröbner basis, this can only happen if $i n_{\omega}\left(g_{i}\right)=i n_{<}\left(g_{i}\right)$. Since $i$ was arbitrary, $i n_{<}\left(g_{i}\right)=i n_{\omega}\left(g_{i}\right)$ for $1 \leq i \leq m$.

For the converse, assume $i n_{<}\left(g_{i}\right)=i n_{\omega}\left(g_{i}\right)$ for $1 \leq i \leq m$. Since

$$
i n_{<}(I)=\left(i n_{<}\left(g_{1}\right), \ldots i n_{<}\left(g_{m}\right)\right)=\left(i n_{\omega}\left(g_{1}\right), \ldots i n_{\omega}\left(g_{m}\right)\right),
$$

it suffices to prove that $i n_{\omega}\left(g_{1}\right), \ldots, i n_{\omega}\left(g_{m}\right)$ generate $i n_{\omega}(I)$. Let $f \in I$. There exist $h_{j} \in R$, $1 \leq j \leq m$ such that $f=\sum_{j=1}^{m} h_{j} g_{j}$, since $G$ is a Gröbner basis for $I$. Consider the weighted degrees of the terms of $f$. For each $h_{j} g_{j}$ the terms with largest weighted degree are $i n_{\omega}\left(h_{j}\right) i n_{\omega}\left(g_{j}\right)$. The terms of $f$ of largest weighted degree will be a sum of such terms unless, the terms involving $i n_{\omega}\left(g_{j}\right)=i n_{<}\left(g_{i}\right)$ cancel for all $1 \leq j \leq m$. However, if this cancellation happens then no term of $f$ involves $i n_{<}\left(g_{j}\right)$ for any $1 \leq j \leq m$, which implies $i n_{<}(f) \notin i n_{<}(I)$, a contradiction. Therefore $i n_{\omega}(f) \in\left(i n_{\omega}\left(g_{1}\right), \ldots, i n_{\omega}\left(g_{m}\right)\right)$.

For $\omega \in \mathbb{N}^{r}$ and $f \in k\left[x_{1}, \ldots, x_{r}\right]$, we define the weighted degree of $f$ to be

$$
\max \left\{\alpha \cdot \omega \mid x_{1}^{\alpha_{1}} \cdots x_{r}^{\alpha_{r}} \text { is a term of } f\right\} .
$$

Let ${ }^{\omega} f=f\left(x_{1} / t^{\omega_{1}}, \ldots x_{r} / t^{\omega_{r}}\right) t^{s}$ where $s$ is minimal such that ${ }^{\omega} f \in k\left[x_{1}, x_{2}, \ldots, x_{r}, t\right]$, then the number $s$ is equal to the weighted degree of $f$. Call ${ }^{\omega} f$ the quasi-homogenization of $f$. Let ${ }^{\omega} I=$ $\left(\left\{{ }^{\omega} f: f \in I\right\}\right)$ be the quasi-homogenization of the ideal $I$.

The quasi-homogenizations of an element $f$ and an ideal $I$ behave as expected with respect to homogenizing and de-homogenizing. We include a few equations to illustrate this. Let $f \in I$. Then ${ }^{\omega} f\left(x_{1}, \ldots, x_{r}, 1\right)=f\left(x_{1} / 1^{\omega_{1}}, \ldots x_{r} / 1^{\omega_{r}}\right) 1^{s}=f\left(x_{1}, \ldots, x_{r}\right)$ and ${ }^{\omega} f \in{ }^{\omega} I$.

Let $F\left(x_{1}, \ldots, x_{r}, t\right) \in{ }^{\omega} I$. Write $F$ as $t^{l} G\left(x_{1}, \ldots, x_{r}, t\right)$ where $t$ does not divide $G$. Let $s$ denote the weighted degree of $G\left(x_{1}, \ldots, x_{r}, 1\right)$. Since $t$ does not divide $G\left(x_{1}, \ldots, x_{r}, t\right),{ }^{\omega} G\left(x_{1}, \ldots, x_{r}, 1\right)=$ $G\left(x_{1} / t^{\omega_{1}}, \ldots, x_{r} / t^{\omega_{r}}, 1\right) t^{s}=G\left(x_{1}, \ldots, x_{r}, t\right)$, and

$$
\begin{array}{cc}
t^{l}\left({ }^{\omega} F\left(x_{1}, \ldots, x_{r}, 1\right)\right)= & t^{l}\left({ }^{\omega} G\left(x_{1}, \ldots, x_{r}, 1\right)\right) \\
=t^{l} G\left(x_{1}, \ldots, x_{r}, t\right)= & F\left(x_{1}, \ldots, x_{r}, t\right) .
\end{array}
$$

If $F \in{ }^{\omega} I$ then there exist $f_{1}, \ldots, f_{m} \in I$ and $r_{1}, \ldots, r_{m} \in k\left[x_{1}, x_{2}, \ldots, x_{r}, t\right]$ such that $F=$ $\sum_{i=1}^{m} r_{i}{ }^{\omega} f_{i}$. Then

$$
\begin{aligned}
F\left(x_{1}, \ldots, x_{r}, 1\right) & =\sum_{i=1}^{m} r_{i}\left(x_{1}, \ldots, x_{r}, 1\right)\left({ }^{\omega} f_{i}\left(x_{1}, \ldots, x_{r}, 1\right)\right) \\
& =\sum_{i=1}^{m} r_{i}\left(x_{1}, \ldots, x_{r}, 1\right) f_{i}\left(x_{1}, \ldots, x_{r}\right) \in I .
\end{aligned}
$$

Lemma 7.3. Let $P$ be an ideal in $k\left[x_{1}, \ldots, x_{r}\right]$ and $<$ any term order. Let $\omega \in \mathbb{N}^{r}$ represent $<$ for $P$ and extend $\omega$ and $<$ to $k\left[x_{1}, x_{2}, \ldots, x_{r}, t\right]$ by taking $x_{i}>t$ for $1 \leq i \leq r$ and give $t$ weight 1 . Set $d=\operatorname{dim}\left(k\left[x_{1}, \ldots, x_{r}\right] / P\right)$. Then

(1) The ideal $P$ is a prime ideal in $R=k\left[x_{1}, \ldots, x_{r}\right]$ if and only if ${ }^{\omega} P$ is a prime ideal in $R[t]$.

(2) If $\left\{g_{1}, \ldots, g_{m}\right\}$ is a Gröbner basis for $P$ then $\left\{{ }^{\omega} g_{1}, \ldots,{ }^{\omega} g_{m}\right\}$ is a Gröbner basis for ${ }^{\omega} P$ and $\operatorname{dim}\left(k\left[x_{1}, x_{2}, \ldots, x_{r}, t\right] / \omega P\right)=d+1$.

(3) ([22], Lemma 3) If $P$ is a prime ideal then in ${ }_{<}(P)+(t)={ }^{\omega} P+(t)$.

Proof. (1): First we prove that ${ }^{\omega}(f g)=\left({ }^{\omega} f\right)\left({ }^{\omega} g\right)$. Let $q, r, s$ denote the weighted total degrees of $f, g$ and $f g$ respectively so $s=r+q$. Then by the definition of quasi-homogenization, ${ }^{\omega} f=$ $f\left(x_{1} / t^{\omega_{1}}, \ldots x_{r} / t^{\omega_{r}}\right) t^{q},{ }^{\omega} g=g\left(x_{1} / t^{\omega_{1}}, \ldots x_{r} / t^{\omega_{r}}\right) t^{r}$ and ${ }^{\omega}(f g)=(f g)\left(x_{1} / t^{\omega_{1}}, \ldots x_{r} / t^{\omega_{r}}\right) t^{s}$. Then

$$
\begin{array}{cc}
\omega(f g) & =(f g)\left(x_{1} / t^{\omega_{1}}, \ldots x_{r} / t^{\omega_{r}}\right) t^{s} \\
=f\left(x_{1} / t^{\omega_{1}}, \ldots x_{r} / t^{\omega_{r}}\right) t^{q} g\left(x_{1} / t^{\omega_{1}}, \ldots x_{r} / t^{\omega_{r}}\right) t^{r} & =\left({ }^{\omega} f\right)\left({ }^{\omega} g\right) .
\end{array}
$$

Assume $P$ is a prime ideal and suppose there exist $F, G \in k\left[x_{1}, x_{2}, \ldots, x_{r}, t\right]$ such that $F G \in{ }^{\omega} P$. Then the equation implies $F\left(x_{1}, \ldots, x_{n}, 1\right) G\left(x_{1}, \ldots, x_{n}, 1\right) \in P$. Since $P$ is a prime ideal, either 
$F\left(x_{1}, \ldots, x_{n}, 1\right) \in P$ or $G\left(x_{1}, \ldots, x_{n}, 1\right) \in P$. Without loss of generality, assume $F\left(x_{1}, \ldots, x_{n}, 1\right) \in$ $P$. Then $F={ }^{\omega} F\left(x_{1}, \ldots, x_{n}, 1\right) t^{s} \in{ }^{\omega} P$, for some $s$. Hence ${ }^{\omega} P$ is a prime ideal.

Conversely, assume ${ }^{\omega} P$ is a prime ideal. Let $f g \in P$. Then $\left({ }^{\omega} f\right)\left({ }^{\omega} g\right)={ }^{\omega}(f g) \in{ }^{\omega} P$. Thus either ${ }^{\omega} f \in{ }^{\omega} P$ or ${ }^{\omega} g \in{ }^{\omega} P$ since ${ }^{\omega} P$ is a prime ideal. Assume ${ }^{\omega} f \in{ }^{\omega} P$, then $f\left(x_{1}, \ldots, x_{r}\right)=$ ${ }^{\omega} f\left(x_{1}, \ldots, x_{r}, 1\right) \in P$ and $P$ is a prime ideal.

(2): Since $\omega$ represents $<$,

$$
i n_{<}\left({ }^{\omega} g_{i}\right)=i n_{<}\left(i n_{\omega}\left(g_{i}\right)\right)=i n_{<}\left(g_{i}\right), \text { for } 1 \leq i \leq m .
$$

Let $F \in{ }^{\omega} P$, then $F\left(x_{1}, \ldots, x_{r}, 1\right) \in P$. Then $F\left(x_{1}, \ldots, x_{r}, 1\right)=\sum_{i=1}^{m} h_{i} g_{i}$ for some $h_{1}, \ldots, h_{m} \in$ $k\left[x_{1}, \ldots, x_{r}\right]$, since $g_{1}, \ldots, g_{m}$ form a Gröbner basis for $P$. Thus, for some $s \in \mathbb{N}, F\left(x_{1}, \ldots, x_{r}, t\right)=$ $t^{s}\left({ }^{\omega} F\left(x_{1}, \ldots, x_{r}, 1\right)\right)=t^{s}\left({ }^{\omega}\left(\sum_{i=1}^{m} h_{i} g_{i}\right)\right)$. Then

$$
i n_{<}(F)=t^{s} i n_{<}\left({ }^{\omega} F\left(x_{1}, \ldots, x_{r}, 1\right)\right)=t^{s} i n_{<}\left({ }^{\omega}\left(\sum_{i=1}^{m} h_{i} g_{i}\right)\right) .
$$

Since $\omega$ represents $<, i n_{<}\left(\sum_{i=1}^{m} h_{i} g_{i}\right)=i n_{<}\left(i n_{\omega}\left(\sum_{i=1}^{m} h_{i} g_{i}\right)\right)$. Therefore the leading term of $\sum_{i=1}^{m} h_{i} g_{i}$ has largest weighted degree. This implies that in ${ }^{\omega}\left(\sum_{i=1}^{m} h_{i} g_{i}\right)$ this term has no $t$ 's in it and is therefore the leading term of ${ }^{\omega}\left(\sum_{i=1}^{m} h_{i} g_{i}\right)$. Hence, using the above equation, $i n_{<}\left({ }^{\omega}\left(\sum_{i=1}^{m} h_{i} g_{i}\right)\right)=$ $n\left(i n_{<}\left(g_{i}\right)\right)=n\left(i n_{<}\left({ }^{\omega} g_{i}\right)\right)$ for some monomial $n \in k\left[x_{1}, \ldots, x_{r}\right]$ and for some $i$. Thus $i n\left({ }^{\omega} P\right)=$ $\left(i n\left({ }^{\omega} g_{1}\right), \ldots i n\left({ }^{\omega} g_{m}\right)\right)$.

Let $R=k\left[x_{1}, \ldots, x_{r}\right]$ and $S=k\left[x_{1}, x_{2}, \ldots, x_{r}, t\right]$. The proof above and the fact that $\operatorname{dim}(R / P)=$ $\operatorname{dim}(R / \operatorname{in}(P))$ imply

$$
\operatorname{dim}\left(S /{ }^{\omega} P\right)=\operatorname{dim}\left(S / \operatorname{in}\left({ }^{\omega} P\right)\right)=\operatorname{dim}(S / \operatorname{in}(P) S)=\operatorname{dim}(R / P)+1=d+1
$$

(3): ([22], Lemma 3$)^{7}$ Let $G$ denote the reduced Gröbner basis for $P$ with respect to $<$. Let $g \in G$. Since $\omega$ represents $<$, for every $g \in G$, $i n_{<}(g)=i n_{\omega}(g)$. Hence ${ }^{\omega} g=i n_{<}(g)+h$ where $h \in(t) k\left[x_{1}, x_{2}, \ldots, x_{r}, t\right]$. Therefore $i n_{<}(g)={ }^{\omega} g-h \in{ }^{\omega} P+(t)$ and $i n_{<}(P) \subseteq{ }^{\omega} P+(t)$. Moreover, ${ }^{\omega} g=i n_{<}(g)+h \in i n_{<}(P)+(t)$ so ${ }^{\omega} P \subseteq i n_{<}(P)+(t)$.

7.3. The Main Theorem. First we restate the connectedness theorem, then we prove a lemma that follows from standard results, but is key to our proof of the theorem of Kalkbrenner and Sturmfels.

Theorem 7.4. (Connectedness Theorem, Theorem 6.4) Let $(R, m, k)$ be an analytically irreducible local ring of dimension $n$, and let $\mathfrak{A}$ be an ideal of $R$ generated by at most $n-2$ elements. Then the punctured spectrum of Spec $R / \mathfrak{A}$ is connected.

LEMma 7.5. Let $R$ be a positively graded Noetherian domain. Assume $R=R_{0}\left[g_{1}, \ldots, g_{m}\right]$ and set $\omega_{i}=\operatorname{deg}\left(g_{i}\right) \geq 1$ for $1 \leq i \leq m$. If $m=\left(g_{1}, \ldots, g_{m}\right)$ denotes the irrelevant ideal, then the $m$-adic completion of $R, \widehat{R^{m}}$, is a domain as well.

Proof. Let $I_{n}$ be the ideal generated by all the homogeneous forms of degree greater than or equal to $n, I_{n}=\left(f \in R_{j} \mid j \geq n\right)$. By construction, $I_{0}=R$ and $I_{n+1} \subseteq I_{n}$. Let $a \in I_{n}$ and $b \in I_{m}$. Each term of $a b$ has degree greater than or equal to $n+m$ which implies $a b \in I_{n+m}$ and hence $I_{n} I_{m} \subseteq I_{n+m}$. Consequently, $\left\{I_{n}\right\}_{n \geq 0}$ is a filtration. Let $\mathfrak{F}$ denote the filtration. We want to prove the following.

(1) $R \simeq g r_{\mathfrak{F}}(R)$.

(2) $g r_{\mathfrak{F}}(R) \simeq g r_{\mathfrak{F}}\left(\widehat{R^{\mathfrak{F}}}\right)=\oplus_{n \geq 0}\left(I_{n} \widehat{R^{\mathfrak{F}}} / I_{n+1} \widehat{R^{\mathfrak{F}}}\right)$.

(3) If $g r_{\mathfrak{F}}(R)=\oplus_{n \geq 0}\left(I_{n} / I_{n+1}\right)$ is a domain then $R$ is a domain.

${ }^{7}$ In $[\mathbf{2 2}]$ the proof given includes many of the properties for homogenization collected above. Hence the proof given here is much shorter while being fundamentally the same proof. 
(4) $\widehat{R^{\mathfrak{F}}} \simeq \widehat{R^{m}}$.

If $R$ is a domain (1) implies $g r_{\mathfrak{F}}(R)$ is a domain and then (2) implies $g r_{\mathfrak{F}}\left(\widehat{R^{\mathfrak{F}}}\right)$ is also a domain. Using (3), since $g r_{\mathfrak{F}}\left(\widehat{R^{\mathfrak{F}}}\right)$ is a domain, $\widehat{R^{\mathfrak{F}}}$ is a domain. Hence by $(4) \widehat{R^{m}}$ is a domain and it is enough to prove (1)-(4).

(1): [4, III.2 no. 3 Example 5].

(2): [30, page 93$]$.

(3): Since $\cap_{n \geq 0} I_{n}=0$, the proof follows that given in [2, Lemma 11.23].

(4): It is enough to prove that the filtrations $\left\{I_{n}\right\}$ and $\left\{m^{n}\right\}$ are cofinal. Let $j$ be given. $I_{j}$ is generated by all the homogeneous elements of $R$ of degree greater than or equal to $j$ and $m^{j}$ is generated by products of the form $g_{1}^{\alpha_{1}} \cdots g_{m}^{\alpha_{m}}$ where $\alpha_{1}+\cdots+\alpha_{m}=j$. The degree of such a product is $\alpha_{1} \omega_{1}+\cdots+\alpha_{m} \omega_{m} \geq j$ and hence $m^{j} \subseteq I_{j}$. Set $W=\max \left\{\omega \cdot \alpha \mid \alpha_{1}+\cdots+\alpha_{m}=j\right\}$, then any homogeneous element of degree greater than or equal to $W$ is in $m^{j}$. Hence $I_{W} \subseteq m^{j}$. Therefore the two filtrations are co-final and $\widehat{R^{\mathfrak{F}}} \simeq \widehat{R^{m}}$.

TheOREM 7.6. [22] Let $P \subseteq R=k\left[x_{1}, \ldots, x_{r}\right]$ be a prime ideal and $<$ any monomial order. Then $R / \sqrt{i n_{<}(P)}$ is equidimensional and connected in codimension one.

Proof. Let $Q_{1}, \ldots Q_{s}$ be the minimal primes of $\operatorname{in}(P)$. For purity, since $R$ is regular, it is enough to prove that $\operatorname{dim}\left(R / Q_{i}\right)=\operatorname{dim}\left(R / Q_{j}\right)$ for all $i \neq j$. Since $Q_{i} \subseteq k\left[x_{1}, \ldots, x_{r}\right]$ is minimal prime ideal of $\operatorname{in}(P),\left(Q_{i}, t\right)$ is a prime ideal minimal over $(i n(P), t)$ for $1 \leq i \leq s$. Hence, by Lemma 7.3, part $(3),\left(Q_{i}, t\right)$ is minimal over $\left({ }^{\omega} P, t\right)$ for $1 \leq i \leq s$. The prime ideals minimal over $(\bar{t})$ in $R /{ }^{\omega} P$ are codimension exactly one. Therefore $\overline{\left(Q_{i}, t\right)}$ is codimension exactly one in $R /{ }^{\omega} P$ for $1 \leq i \leq s$. Hence $\operatorname{codim}\left(Q_{i}, t\right)=\operatorname{codim}\left(Q_{j}, t\right)$ for $1 \leq i, j \leq s$. Since $R$ is a Cohen-Macaulay ring, $\operatorname{codim}\left(Q_{i}, t\right)=\operatorname{codim}\left(Q_{j}, t\right)$ implies

$$
\operatorname{dim}\left(k\left[x_{1}, x_{2}, \ldots, x_{r}, t\right] /\left(Q_{i}, t\right)\right)=\operatorname{dim}\left(k\left[x_{1}, x_{2}, \ldots, x_{r}, t\right] /\left(Q_{j}, t\right)\right) .
$$

Also, $Q_{i} \subseteq k\left[x_{1}, \ldots, x_{r}\right]$, for $1 \leq i \leq s$ implies

$$
k\left[x_{1}, x_{2}, \ldots, x_{r}, t\right] /\left(Q_{i}, t\right) \simeq k\left[x_{1}, \ldots, x_{r}\right] / Q_{i}, \text { for } 1 \leq i \leq s .
$$

Thus $\operatorname{dim}\left(k\left[x_{1}, \ldots, x_{r}\right] / Q_{i}\right)=\operatorname{dim}\left(k\left[x_{1}, \ldots, x_{r}\right] / Q_{j}\right)$, for $1 \leq i, j \leq s$.

We prove that $R / \sqrt{\operatorname{in}(P)}$ is connected in codimension one. Let $S=k\left[x_{1}, x_{2}, \ldots, x_{r}, t\right]$ and $m=\left(x_{1}, \ldots, x_{r}, t\right)$. Since $\left(i n\left({ }^{\omega} P\right), t\right)=(i n(P), t)=\left({ }^{\omega} P, t\right)$ we can assume $P \subseteq S$ is a quasihomogeneous prime ideal, $t \notin P, \operatorname{in}(P) \subseteq k\left[x_{1}, \ldots, x_{r}\right]=R$ and $(i n(P), t)=(P, t)$. Let $Q_{1}, \ldots Q_{s}$ be the prime ideals minimal over $i n(P)$. By purity $\operatorname{dim}\left(R / Q_{j}\right)=\operatorname{dim}\left(R / Q_{j}\right)=d$ for $1 \leq i, j \leq s$. The ideal $Q_{i}$ is a prime ideal minimal over a monomial ideal, so it is generated by a subset $Y_{i} \subseteq$ $\left\{x_{1}, \ldots, x_{r}\right\}$ of cardinality $r-d$. Let $l_{1}, \ldots, l_{d-2} \in\left(x_{1}, \ldots, x_{r}, t\right)=m$ be linear forms such that $\operatorname{codim}\left(\left(Y_{i}, Y_{j}, t, l_{1}, \ldots, l_{d-2}\right)\right)=\min \left\{\operatorname{codim}\left(\left(Y_{i}, Y_{j}\right)\right)+1+d-2, r+1\right\}$ for $1 \leq i \neq j \leq s$ (such linear forms exist if $k$ is infinite, e.g. $k$ is algebraically closed). Let $\underline{l}=\left\{l_{1}, \ldots, l_{d-2}\right\}$.

By the choice of $\underline{l}$, the following are equivalent.

(1) $\operatorname{codim}\left(\left(Y_{i}, Y_{j}, t, \underline{l}\right)\right)=r$

(2) $\left|Y_{i} \backslash Y_{j}\right|=1$

(3) $\left(Y_{i}, Y_{j}, t, \underline{l}\right) \neq m$

(4) $V\left(Y_{i}, t, \underline{l}\right) \cap V\left(Y_{j}, t, \underline{l}\right) \neq \emptyset$.

Therefore $\cup_{i=1}^{s} V\left(Y_{i}, t, \underline{l}\right)=\left(\cup_{i=1}^{s} V\left(Y_{i}\right)\right) \cap V(t, \underline{l})$ connected in projective space implies $R / \sqrt{\operatorname{in}(P)}$ is connected in codimension one. Since the variety $\cup_{i=1}^{s} V\left(Y_{i}, t, \underline{l}\right)$ corresponds to the set of prime ideals containing $(\operatorname{in}(P), t, \underline{l}) \subseteq m$ minus the maximal ideal, it is enough to prove the space

$$
\operatorname{Spec}\left(\frac{S_{m}}{(i n(P), t, \underline{l})}\right) \backslash\left\{\frac{m}{(i n(P), t, \underline{l})}\right\} \text {. }
$$


is connected.

By Lemma 7.3 and the fact that $P$ is quasi-homogeneous, so $P \subseteq m$,

$$
\frac{S_{m}}{(i n(P), t, \underline{l})}=\frac{S_{m}}{(P, t, \underline{l})} .
$$

Hence we can consider the connectedness of

$$
\operatorname{Spec}\left(\frac{S_{m}}{(P, t, \underline{l})}\right) \backslash\left\{\frac{m}{(P, t, \underline{l})}\right\} \text {. }
$$

By Lemma $7.5 \widehat{\left(\frac{S_{m}}{P}\right)}$ is a domain of dimension $d+1$ since $P \subseteq S$ is a quasi-homogeneous prime ideal and therefore $\frac{S_{m}}{P}$ is analytically irreducible. The sequence $t, \underline{l}$ lies in $m$ and has length $d-1 \leq$ $(d+1)-2$ so the connectedness theorem states that

$$
\operatorname{Spec}\left(\frac{S_{m}}{(P, t, \underline{l})}\right) \backslash\left\{\frac{m}{(P, t, \underline{l})}\right\} .
$$

is connected. $\operatorname{Spec}\left(\frac{S_{m}}{(i n(P), t, \underline{l})}\right) \backslash\left\{\frac{m}{(i n(P), t, \underline{l})}\right\}$ is connected as needed.

\section{Appendix 2: Bass numbers and Gorenstein Rings}

As a first step, we desire a criterion to allow us to check whether a given injective resolution is minimal. To obtain such a criterion, it will suffice to give a homological criterion for an extension of $R$-modules to be essential. Throughout this section if $p$ is a prime of a ring $R$, we denote the residue field of $R_{p}$ by $\kappa(p)$.

Proposition 8.1. Suppose that $f: M \longrightarrow N$ is an injective map of $R$-modules. Then $N$ is an essential extension of $f(M)$ if and only if for every $p \in \operatorname{Spec}(R)$, the induced map $f_{p}$ : $\operatorname{Hom}_{R_{p}}\left(\kappa(p), M_{p}\right) \longrightarrow \operatorname{Hom}_{R_{p}}\left(\kappa(p), N_{p}\right)$ is an isomorphism.

Proof. Since localization is exact and $\operatorname{Hom}(\kappa(p), \quad)$ is left exact, $f_{p}$ is always injective. First assume that $N$ is essential over $f(M)$, and let $\gamma \in \operatorname{Hom}_{R_{p}}\left(\kappa(p), N_{p}\right)$. Since $R / p$ is finitely presented, $\gamma$ is induced, up to a unit of $R_{p}$, by some $\varphi \in \operatorname{Hom}_{R}(R / p, N)$. Set $x=\varphi(1)$. Let $E$ be an injective hull of $M$. Then $E$ is essential over $M$ so that there exists an $r \in R$ with $r x \in f(M)$ and $r x \neq 0$. However $r \notin p$ since $p x=0$. Therefore $\gamma(1) \in f(M)_{p}$ which shows that $f_{p}$ is surjective, and therefore an isomorphism. Next suppose that $f_{p}$ is always an isomorphism, and let $x \in N$. Choose any $p \in \operatorname{Ass}(R x)$; then there is a $y=r x$ such that $\operatorname{ann}_{R} y=p$. Define a homomorphism $\gamma \in \operatorname{Hom}_{R}(R / p, N)$ by $\gamma(1)=y$. Then by assumption $\gamma_{p}$, the map induced from $\gamma$ by localizing at $p$, is induced by a homomorphism of $\kappa(p)$ to $M_{p}$. This implies that $y / 1 \in f\left(M_{p}\right)$, and thus there exists an $s \notin p$ such that $s y \in f(M)$. Since $\operatorname{ann}_{R} y=p, s y \neq 0$, which proves that $N$ is essential over $f(M)$.

COROLlary 8.2. Let $R$ be a Noetherian ring and $M$ an $R$-module with injective resolution $E^{*}$. Then $E^{*}$ is a minimal injective resolution if and only if for all $p \in \operatorname{Spec}(R)$, and all $i$, the induced maps $\operatorname{Hom}_{R_{p}}\left(\kappa(p), E_{p}^{i}\right) \longrightarrow \operatorname{Hom}_{R_{p}}\left(\kappa(p), E_{p}^{i+1}\right)$ are zero.

Proof. Let $Z^{i}=\operatorname{ker}\left(\varphi_{i+1}\right)=\operatorname{Im}\left(\varphi_{i}\right) . \quad E^{*}$ is minimal if and only if $E^{i}$ is an injective hull of $Z^{i}$, if and only if $E^{i}$ is an essential extension of $Z^{i}$, if and only if the induced maps $\operatorname{Hom}_{R_{p}}\left(\kappa(p),\left(Z^{i}\right)_{p}\right) \longrightarrow \operatorname{Hom}_{R_{p}}\left(\kappa(p),\left(E^{i}\right)_{p}\right)$ are isomorphisms (by Proposition 8.1). Since there are exact sequences $0 \longrightarrow Z^{i} \longrightarrow E^{i} \longrightarrow E^{i+1}$, and $\operatorname{Hom}_{R_{p}}(\kappa(p), \quad)$ is left exact, the latter condition holds if and only if the induced maps of the statement of the corollary are isomorphisms. 
COROLlary 8.3. If $R$ is a Noetherian ring and $M$ is an $R$-module with a minimal injective resolution $E^{*}$, then for all $p \in \operatorname{Spec}(R), E_{p}^{*}$ is a minimal injective resolution of $M_{p}$.

Proof. We know that each $E_{p}^{i}$ is injective as an $R_{p}$-module. Therefore we may apply Corollary 8.2. We need to show that the induced maps $\operatorname{Hom}_{R_{q}}\left(\kappa(q),\left(E_{p}^{i}\right)_{q}\right) \longrightarrow \operatorname{Hom}_{R_{q}}\left(\kappa(q),\left(E_{p}^{i+1}\right)_{q}\right)$ is zero for all $q \subset p$. Since $\left(E_{p}^{i}\right)_{q}=E_{q}^{i}$, the minimality of $E^{*}$ together with Corollary 8.2 show that these maps are zero.

Definition 8.4. Let $R$ be a Noetherian ring and let $M$ be any $R$-module with minimal injective resolution $E^{*}$. The Bass numbers, $\mu_{i}(p, M)$, are defined to be the number of copies of $E_{R}(R / p)$ in a direct sum decomposition of $E^{i}$ into indecomposable injective modules. If $R$ is local with maximal ideal $\mathfrak{m}$, then we let $\mu_{i}(M)=\mu_{i}(\mathfrak{m}, M)$.

REMARK 8.5. The Bass numbers do not depend upon the minimal injective resolution chosen, nor upon the particular direct sum decomposition of $E^{i}$.

COROLlary 8.6. Let $R$ be a Noetherian ring and let $M$ be an $R$-module. Then $\mu_{i}(p, M)=$ $\mu_{i}\left(p_{p}, M_{p}\right)$ for all $i$.

Proof. Fix a minimal injective resolution $E^{*}$ of $M$. By Corollary 8.3, $E_{p}^{*}$ is a minimal injective resolution of $M_{p}$. Write $E^{1}=\oplus E_{R}(R / p)^{\mu_{i}(p, M)}$. It suffices to see that $\left(E_{R}(R / q)\right)_{p} \cong E_{R}\left(R_{p} / q_{p}\right)$ for $q \subset p$, and is equal to 0 if $q$ is not contained in $p$, which is easy.

TheOREM 8.7. Let $R$ be a Noetherian ring and $M$ an $R$-module. Then

$$
\mu_{i}(p, M)=\operatorname{dim}_{\kappa(p)}\left(\operatorname{Ext}^{i}\left(\kappa(p), M_{p}\right)\right) .
$$

In particular, if $M$ is finitely generated, all of the Bass numbers are finite.

Proof. By Corollary 8.6 it suffices to treat the case where $(R, \mathfrak{m}, k)$ is a Noetherian local ring and $p=\mathfrak{m}$. Let $E^{*}$ be a minimal injective resolution of $M$. Then $\operatorname{Ext}^{i}(k, M)$ is the $i$ th homology of the complex $\operatorname{Hom}_{R}\left(k, E^{*}\right)$. By the minimality of the resolution all maps in this complex are zero, so that $\operatorname{Ext}^{i}(k, M)=\operatorname{Hom}_{R}\left(k, E^{i}\right)$. To finish the proof it suffices to show that $\operatorname{Hom}_{R}\left(k, E_{R}(R / p)\right)=0$ if $p \neq \mathfrak{m}$, and is $k$ if $p=\mathfrak{m}$. The latter statement follows since $\operatorname{Hom}_{R}\left(k, E_{R}(k)\right) \cong E_{k}(k)=k$, by . If $p \neq \mathfrak{m}$, then there is an element $x$ of $\mathfrak{m}$ not in $p$, and this element acts as a unit on $E_{R}(R / p)$. Any element $\varphi$ in $\operatorname{Hom}_{R}\left(k, E_{R}(R / p)\right)$ must send 1 to an element annihilated by $x$, and thus 0 .

REMARK 8.8. It is a remarkable fact that if $R$ is a regular local ring containing a field, or unramified in mixed characteristic, and $I$ is an ideal of $R$, then the Bass numbers of the local cohomology modules $H_{I}^{i}(R)$ are finite, even though these modules are in general infinitely generated. This fact was proved first in positive characteristic in [21], in equicharacteristic 0 in [25], and in the unramified mixed characteristic case in $[\mathbf{2 7}]$. See [26] for a survey on this topic as well as others related to these notes.

Definition 8.9. Let $R$ be a ring and let $M$ be an $R$-module. The injective dimension of $M$, denoted by $\operatorname{id}_{R} M$, is the length of a minimal injective resolution of $M$. A local Noetherian ring $R$ is said to be Gorenstein if $\operatorname{id}_{R}(R)<\infty$.

Later we will prove that the definition here, which is the usual one, agrees with the definition of Gorenstein found in the main text.

We wish to prove several fundamental facts concerning Gorenstein rings, among them the fact that they are always Cohen-Macaulay. First we will do the case where the dimension of $R$ is 0 .

Proposition 8.10. Let $(R, \mathfrak{m}, k)$ be a 0-dimensional Noetherian local ring. Then the following are equivalent: 
(1) $R$ is Gorenstein.

(2) $\operatorname{id}_{R}(R)=0$.

(3) $R \cong E_{R}(k)$.

(4) $\operatorname{dim}_{k}(\operatorname{socle}(R))=1($ here $\operatorname{socle}(N)=\{x \in N: \mathfrak{m} x=0\})$.

Proof. Clearly (2) and (3) are equivalent and imply (1). Suppose (1) holds. Since $E=E_{R}(k)$ is the only indecomposable injective module, $R$ has a minimal injective reslution of the form:

$$
0 \longrightarrow R \longrightarrow E^{b_{1}} \longrightarrow \ldots \longrightarrow E^{b_{n}} \longrightarrow 0 \text {. }
$$

Apply ${ }^{\vee}=\operatorname{Hom}_{R}(, E)$ to this exact sequence. We obtain an exact sequence,

$$
0 \longrightarrow R^{b_{n}} \longrightarrow \ldots \longrightarrow R^{b_{1}} \longrightarrow E \longrightarrow 0
$$

Hence $\operatorname{pd}_{R}(E)<\infty$. By the Auslander-Buchsbaum formula it follows that $E$ is free. Since $E$ is indecomposable, we obtain that $E \cong R$, which proves (3) and (2).

Now assume (3). By Proposition 3.9, $\lambda\left(k^{\vee}\right)=\lambda(k)=1$, so that $\lambda\left(\operatorname{Hom}_{R}(k, E)\right)=1$. Since $R \cong E, \lambda\left(\operatorname{Hom}_{R}(k, R)\right)=1$. This Hom is naturally identified with socle $(R)$, which proves $(4)$.

Finally assume that (4) holds. We claim that $R$ is an essential extension of $\operatorname{socle}(R)$. For if $I$ is any ideal of $R, \mathfrak{m}^{n} I=0$ for some $n$; if we choose such an $n$ least, then $\mathfrak{m}^{n-1} I \subset \operatorname{socle}(R) \cap I$ and is nonzero. If $\operatorname{socle}(R) \cong k$, then $R$ is an essential extension of $k$, and therefore embeds in $E$. By Proposition $3.9, \lambda(R)=\lambda(E)$, so that $R \cong E$.

Clearly if $\operatorname{id}_{R}(M)<\infty$, then the injective dimension is characterized by the last nonvanishing Bass number. We wish to show that it is even characterized by the last nonvanishing Bass number for the maximal ideal. To prove this we require a fundamental lemma.

Lemma 8.11. Let $R$ be a Noetherian ring and let $M$ be a finitely generated $R$-module. Let $p \subset q$ be prime ideals of $R$ with $\operatorname{ht}(q / p)=s$. If $\mu_{i}(p, M) \neq 0$, then $\mu_{i+s}(q, M) \neq 0$.

Proof. By induction on $s$, it suffices to treat the case where $s=1$. Next we may localize at $q$ to assume that $q=\mathfrak{m}$, the maximal ideal of $R$, since the Bass numbers do not change under localization. Suppose that $\mu_{i+1}(\mathfrak{m}, R)=0$. By Theorem 8.7, $\operatorname{Ext}_{R}^{i+1}(k, R)=0$. By an easy induction, we then obtain that $\operatorname{Ext}_{R}^{i+1}(L, R)=0$ for any module $L$ of finite length. Now choose an $x \notin p$, and consider the short exact sequence,

$$
0 \longrightarrow R / p \stackrel{x}{\longrightarrow} R / p \longrightarrow R /(p, x) \longrightarrow 0
$$

Applying $\operatorname{Hom}(\quad, R)$ and using that $R /(p, x)$ has finite length we see that $\operatorname{Ext}_{R}^{i}(R / p, R)=x \operatorname{Ext}_{R}^{i}(R / p, R)$, so that by Nakayama's lemma this Ext is 0 . Therefore $\mu_{i}(p, R)=0$, by Theorem 8.7 , which proves the lemma.

LEMMA 8.12. Let $(R, \mathfrak{m}, k)$ be a Noetherian local ring and suppose that $x \in \mathfrak{m}$ is a nonzerodivisor (NZD) in $R$. Let $E^{*}$ be a minimal injective resolution of $R$. Then $\operatorname{Hom}_{R}\left(R / R x, E^{i}\right)_{i \geq 1}$ is a minimal injective resolution of $R / R x$ as an $R / R x$-module.

Proof. The modules $C^{i-1}=\operatorname{Hom}_{R}\left(R / R x, E^{i}\right)$ are injective $R / R x$-modules. We claim that $\operatorname{Hom}_{R}\left(R / R x, E^{0}\right)=0$. This will follow if we show that $x$ is a NZD on $E^{0}$. If $y \in E^{0}$ and $x y=0$ but $y \neq 0$, then there exists an $r \in R$ with $r y \neq 0$, and $r y \in R$, since $E^{0}$ is an essential extension of $R$. However, $0=r(x y)=x(r y)$ contradicts our assumption. Since this Hom is zero, the kernel of the map from $C^{0}$ to $C^{1}$ is exactly $\operatorname{Ext}_{R}^{1}(R / R x, R) \cong R / R x$ (since $x$ is a NZD). Hence

$$
0 \longrightarrow R / R x \longrightarrow C^{0} \longrightarrow C^{1} \longrightarrow \ldots \longrightarrow C^{n} \longrightarrow \ldots
$$

is an injective resolution of $R / R x$ as an $R / R x$-module, since $\operatorname{Ext}_{R}^{i}(R / R x, R)=0$ for $i \geq 2$, as $\operatorname{pd}_{R}(R / R x)=1$. We must show that this is a minimal injective resolution of $R / R x$. We use the criterion of Corollary 8.2. Let $p \in \operatorname{Spec}(R / R x)$. The map of $\operatorname{Hom}_{(R / R x)_{p}}\left(\kappa(p), C_{p}^{i}\right)$ to 
$\operatorname{Hom}_{(R / R x)_{p}}\left(\kappa(p), C_{p}^{i+1}\right)$ is, after using the Hom-tensor adjoint relationship, simply the map from $\operatorname{Hom}_{R_{p}}\left(\kappa(p), E_{p}^{i}\right)$ to $\operatorname{Hom}_{R_{p}}\left(\kappa(p), E_{p}^{i+1}\right)$ which is zero by the minimality of $E^{*}$.

There are several nice corollaries to this lemma which are very useful.

Corollary 8.13. Let $(R, \mathfrak{m}, k)$ be a Noetherian local ring. Suppose that $x \in \mathfrak{m}$ is a NZD. Then $\operatorname{id}_{R}(R)=\operatorname{id}_{R / R x}(R / R x)+1$.

Proof. Let $E^{*}$ be a minimal injective resolution of $R$ as an $R$-module. Lemma 8.12 shows in any case that $\operatorname{id}_{R}(R) \geq \operatorname{id}_{R / R x}(R / R x)+1$. First suppose that $\operatorname{id}_{R}(R)=n<\infty$. Lemma 8.11 shows that $E^{n}$ must be a direct sum of copies of $E=E_{R}(k)$. Then $\operatorname{Hom}_{R}\left(R / R x, E^{n}\right) \cong \operatorname{Hom}_{R}(R / R x, E)^{n} \cong$ $E_{R / R x}(k)^{n}$ by 1 . In particular, using the notation of Lemma 8.12 we obtain that $C^{n-1} \neq 0$, and so $\operatorname{id}_{R / R x}(R / R x)+1=n$.

If $\operatorname{id}_{R}(R)=\infty$, then again Lemma 8.11 shows that there exist arbitrarily large $n$ for which $\mu_{n}(R) \neq 0$. The same remarks as in the above paragraph then prove that $\mu_{n-1}(R / R x) \neq 0$ (as $R / R x$-module) and so $\operatorname{id}_{R / R x}(R / R x)=\infty$.

Corollary 8.14. Let $(R, \mathfrak{m}, k)$ be a local ring and suppose that $x \in \mathfrak{m}$ is a NZD on $R$. Suppose that $x M=0$. Then $\operatorname{Ext}_{R}^{i}(M, R) \cong \operatorname{Ext}_{R / R x}^{i-1}(M, R / R x)$.

Proof. To compute the first Ext, we can take a minimal injective resolution

THEOREM 8.15. Let $(R, \mathfrak{m}, k)$ be a local Noetherian ring of dimension d. The following conditions are equivalent:

(1) $R$ is Gorenstein.

(2) $\operatorname{id}_{R}(R)=d$.

(3) $R$ is Cohen-Macaulay and if $x_{1}, \ldots, x_{d}$ is any s.o.p. generating an ideal $I$, then $\operatorname{dim}_{k}(\operatorname{socle}(R / I))=$ 1 .

(4) $R$ is Cohen-Macaulay and $H_{\mathfrak{m}}^{d}(R) \cong E_{R}(k)$.

Proof. We first prove that (1), (2), and (3) are equivalent. Clearly (2) implies (1). In general we claim that if $M$ is finitely generated, and $\operatorname{id}_{R}(M)=n<\infty$, then $n \leq \operatorname{depth}(R)$. Assume $t=\operatorname{depth}(R)<n$. Let $x_{1}, \ldots, x_{t}$ be a maximal $R$-sequence generating an ideal $J$. Consider $\operatorname{Ext}_{R}^{n}(R / J, M)$. Since $\operatorname{pd}_{R}(R / J)=t<n$, this module is 0 . On the other hand, $\operatorname{since} \operatorname{depth}(R / J)=$ 0 , there is an exact sequence,

$$
0 \longrightarrow k \longrightarrow R / J \longrightarrow N \longrightarrow 0
$$

Apply $\operatorname{Hom}(, M)$; there is an exact sequence,

$$
\operatorname{Ext}_{R}^{n}(R / J, M) \longrightarrow \operatorname{Ext}_{R}^{n}(k, M) \longrightarrow \operatorname{Ext}_{R}^{n+1}(N, M)
$$

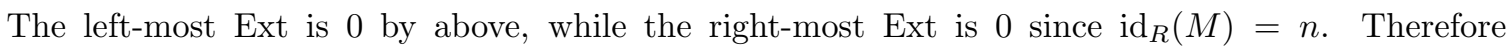
$\operatorname{Ext}_{R}^{n}(k, M)=0$, so that $\mu_{n}(M)=0$, which contradicts the fact that $\operatorname{id}_{R}(M)=n$ (by Lemma 8.11 there must always exist a copy of $E_{R}(k)$ in the last injective module in a finite injective resolution).

On the other hand, $\operatorname{id}_{R}(R)=\max _{i}\left\{\mu_{i}(R) \neq 0\right\}=\max _{i}\left\{\operatorname{Ext}_{R}^{i}(k, R) \neq 0\right\}$, while $\operatorname{depth}(R)=$ $\min _{i}\left\{\operatorname{Ext}_{R}^{i}(k, R) \neq 0\right\}$. Hence $\operatorname{id}_{R}(R) \geq \operatorname{depth}(R)$. Putting this together with the above inequality yields that $\operatorname{id}_{R}(R)=\operatorname{depth}(R)$. Assume $\left.\mathrm{i}\right)$. Let $t=\mathrm{id}_{R}(R)$ and choose a maximal $R$-sequence $x_{1}, \ldots, x_{t}$ in $R$. By Corollary 8.13, letting $S=R /\left(x_{1}, \ldots, x_{t}\right), \operatorname{id}_{S}(S)=\operatorname{id}_{R}(R)-t=0$. Hence $S$ is injective. Since $S$ contains a copy of $k, S$ must contain a copy of $E_{S}(k)$, which must split off from $S$. $S$ is indecomposable and therefore $S=E_{S}(k)$. As $E_{S}(k)$ is Artinian, we obtain that $\operatorname{dim}(S)=0$, and thus $\operatorname{dim}(R)=t=\mathrm{id}_{R}(R)$. This proves $(2)$.

Assume (2). By above $\operatorname{id}_{R}(R)=\operatorname{depth}(R)$, so that (2) implies that $R$ is C-M. Let $x_{1}, \ldots, x_{d}$ be a s.o.p. of $R$. Let $S=R /\left(x_{1}, \ldots x_{d}\right)$. Then $\operatorname{id}_{S}(S)=0$, which shows that $S$ is Gorenstein. Proposition 8.10 gives that $\operatorname{dim}_{k}\left(\operatorname{Hom}_{S}(k, S)\right)=1$, proving $(3)$. 
Finally assume that (3) holds. Let $x_{1}, \ldots, x_{d}$ be a maximal regular sequence in $R$. Let $S=$ $R /\left(x_{1}, \ldots, x_{d}\right)$. By Corollary 8.13 it suffices to see that $S$ is Gorenstein. Since the dimension of socle $(S)$ is 1 by assumption, Proposition 8.10 shows $S$ is Gorenstein.

We next prove (4) is equivalent to (3). For both directions, we may assume that $R$ is complete and Cohen-Macaulay. We use induction on the dimension $d$ of $R$ for both directions. In either direction, the fact that $R$ is Cohen-Macaulay gives us a short exact sequence,

$$
0 \rightarrow H_{\mathfrak{m}}^{d-1}(R / x R) \rightarrow H_{\mathfrak{m}}^{d}(R) \stackrel{x}{\rightarrow} H_{\mathfrak{m}}^{d}(R) \rightarrow 0
$$

where $x$ is any non-zerodivisor of $R$.

First assume that (4) holds. The exact sequence above identifies $H_{\mathrm{m}}^{d-1}(R / x R)$ with the annihlator of $x$ in $H_{\mathfrak{m}}^{d}(R)=E_{R}(k)$. But this annihlator is $\operatorname{Hom}_{R}\left(R / x R, E_{R}(k)\right)=E_{R / R x}(k)$. By induction, $R / x R$ satifies condition (3), and (3) then follows by letting $x=x_{1}$.

Next assume (3). Taking the Matlis duals in the exact sequence above yields a short exact sequence,

$$
0 \rightarrow M \stackrel{x}{\rightarrow} M \rightarrow R / x R \rightarrow 0
$$

where $M$ is the Matlis dual of $H_{\mathfrak{m}}^{d}(R)$, and where by induction we have identified $R / x R$ with the Matlis dual of $H_{\mathfrak{m}}^{d-1}(R / x R)$. This sequence shows that $M / x M \cong R / x R$, and it easily follows that $M \cong R$. Taking Matlis duals again yields that $H_{\mathfrak{m}}^{d}(R)=M^{\vee}=R^{\vee}=E_{R}(k)$, proving (4).

Condition (4) in the above theorem is the "non-standard" definition we gave for a Gorenstein ring. This theorem proves that our definition agrees with the usual ones.

\section{References}

1. J. Alvarez-Montaner, M. Blickle, and G. Lyubeznik, Generators of D-modules in positive characteristic, Math. Rese. Letters 12, 2005, 459-473.

2. M. Atiyah and I. Macdonald, Introduction to Commutative Algebra, Addison-Wesley Publishing Co., New York, 1969.

3. T. Becker and V. Weispfenning, Gröbner Bases, Graduate Texts in Mathematics 141. Springer-Verlag, New York, 1993.

4. N. Bourbaki, Commutative Algebra, Springer-Verlag, New York, 1989.

5. M. Brodmann, Einige Ergebnisse aus der Lokalen Kohomologie theorie und Ihre Anwendung, Osnabrücker Schriffen zur Mathematik, Heft 5, 1983.

6. M. Brodmann and C. Huneke, A quick proof of the Hartshorne-Lichtenbaum vanishing theorem, Algebraic gemoetry and its applications, Springer, New York, 1994, 305-308.

7. M. Brodmann and J. Rung, Local cohomology and the connectedness dimension in algebraic varieties, Comment. Math. Helvetici 61 (1986), 481-490.

8. M. Brodmann and R. Sharp, Local cohomology: an algebraic introduction with geometric applications, Cambridge Studies in Advanced Mathematics, 60 (1998), Cambridge University Press, Cambridge.

9. F. W. Call and G. Lyubeznik, A simple proof of Grothendieck's theorem on the parafactoriality of local rings, Commutative algebra: syzygies, multiplicities, and birational algebra (South Hadley, MA, 1992), Contemp. Math., 159 (1994), 15-18.

10. R. Charney and M. Davis, Euler characteristic of a nonpositively curved, piecewise Euclidean manifold, Pac. J. Math. 171 (1995), 117-137.

11. G. Faltings, Uber lokale Kohomologiegruppen hoher Ordnung, J. fur d. reine u. angewandte Math. 313 (1980), $43-51$.

12. G. Faltings, Some Theorems about Formal Functions, Publ. RIMS Kyoto Univ. 16 (1980 ), 721-737.

13. W. Fulton and J. Hansen, A connectedness theorem for projective varieties, with applications to intersections and singularities of mappings, Annals of Math. 109 (1979), 159-166.

14. A. Grothendieck (notes by R. Hartshorne),Local Cohomology, Springer Lecture Notes in Math., 41, SpringerVerlag, 1966 .

15. A. Grothendieck, Cohomologie locale des faisceaux cohérents et théorèmes de Lefschetz locaux et globaux SGA2, North-Holland, Amsterdam, 1968.

16. R. Hartshorne, Cohomological Dimension of Algebraic Varieties, Annals of Math. 88 (1968). 
17. R. Hartshorne, Ample Subvarieties of Algebraic Varieties, Lecture Notes in Mathematics 156, Springer-Verlag, Berlin-Heidelberg-New York, (1970).

18. M. Hochster, Local cohomology, unpublished notes.

19. C. Huneke and R. Sharp, Bass numbers of local cohomology modules, Trans. Amer. Math. Soc. 339 (1993), 765-779.

20. C. Huneke and G. Lyubeznik, On the vanishing of local cohomology modules, Invent. math.,102 (1990), 73-93.

21. C. Huneke and K.E. Smith, Tight closure and the Kodaira vanishing theorem, J. fur die reine und Angewandte Math., 484 (1997), 127-152.

22. M. Kalkbrenner and B. Sturmfels, Initial complexes of prime ideals, Advances in Math., 116 (1995), 365-376.

23. H. Kredel and V. Weispfenning, Computing dimension and independent sets for polynomial ideal, J. Symbolic Computation, 6 (1988), 210-248.

24. S. Lichtenbaum,

25. G. Lyubeznik, Finiteness properties of local cohomology modules (an application of D-modules to Commutative Algebra), Invent. Math., 113 (1993), 41-55.

26. G. Lyubeznik, Survey of local cohomology in Local Cohomology and its Applications, Lecture Notes in Pure and Applied Mathematics, Marcel Dekker226 (2002).

27. G. Lyubeznik, Finiteness properties of local cohomology modules for regular local rings of mixed characteristic: the unramified case, Comm. Alg. 28 (2000), 5867-5882.

28. E. Matlis, Injective modules over noetherian rings, Pacific J. Math. 8 (1958), 511-528.

29. E. Matlis, Modules with descending chain condition, Transactions Amer. Math. Soc. 97 (1960), 495-508.

30. H. Matsumura, Commutative Ring Theory, Cambridge University Press, New York, 1986.

31. T. Mora and L. Robbiano, The Gröbner fan of an ideal, J. Symbolic Computation, 6 (1988), 183-208.

32. A. Ogus, Local cohomological dimension of algebraic varieties, Annals Math.,98 (1973), 327-365.

33. C. Peskine and L. Szpiro, Dimension projective finie et cohomologie locale, I.H.E.S. 42 (1973), 323-395.

34. V. Reiner and V. Welker, On the Charney-Davis and Neggers-Stanley conjectures, J. Comb. Theory, 109 (2005), 247-280.

35. A. R. Richardson A technique for explicit computation of local cohomology with respect to monomial ideals, preprint.

36. E. H. Spanier, Algebraic Topology, McGraw-Hill, New York, 1966.

37. R. Stanley, Combinatorics and Commutative Algebra, Birkhäuser, Boston, 1983.

38. B. Sturmfels Gröbner Bases and Convex Polytopes, AMS University Lecture Series, vol. 8, American Mathematical Society, Providence, RI, 1996.

39. I. Swanson, A note on analytic spread Comm. Algebra 22 (1994), 407-411.

40. U. Walther, Algorithmic computation of local cohomology and the local cohomological dimension of algebraic varieties, J. Pure Appl. Alg., 139 (1999), 303-321.

Department of Mathematics, University of Kansas, Lawrence, KS 66045

E-mail address: huneke@math.ku.edu

URL: http://www.math.ku.edu/ huneke

Department of Mathematics, St. Olaf College, 1520 St. Olaf Ave., Northfield, MN 55057

E-mail address: ataylor@stolaf.edu 